\title{
Optical Co-orbital Measurements in Low Earth Orbit
}

\author{
by \\ Kevin Bernard \\ A thesis submitted to the Faculty of Graduate and Postdoctoral Affairs \\ in partial fulfillment of the requirements for the degree of

\section{Master of Applied Science in \\ Aerospace Engineering} \\ Carleton University \\ Ottawa, Ontario \\ (C) 2019 \\ Kevin Bernard
}


For all the support my family has provided over the years, this work is dedicated to them. 


\begin{abstract}
The increasing access to the space domain has led to the growth of the on-orbit object population, as well as the evolution of space surveillance capabilities. With the increased public attention paid to spacecraft proximity and rendezvous operations, is an opportunity to examine what and how current space surveillance capabilities can contribute to this field.

This thesis examines the feasibility and challenges associated with re-purposing an existing on-orbit space surveillance capability, the NEOSSat microsatellite, to perform proximity operations. The ability of NEOSSat to collect a sufficient number of accurate optical observations to determine the relative orbit of a target spacecraft is explored. A variety of observation events are explored with a collection of spacecraft to generate relative orbits using inertial orbit determination techniques. These practical results, to the author's knowledge, are the first in the field of relative orbit determination using inertial techniques between two co-orbital Low Earth Orbit satellites.
\end{abstract}




\section{Acknowledgements}

I would like to sincerely thank my supervisors, Dr. Steve Ulrich and Dr. Lauchie Scott. Thank you Steve for providing guidance, and for taking a chance on someone who walked in to your office in the Spring of 2016. Thank you Lauchie for supporting this work with access to NEOSSat, and especially for convincing me that I should.

I would also like to express my gratitude to Stefan Thorsteinson for the insights he provided on NEOSSat image processing, as well as taking the time to produce the required NEOSSat schedules to observe satellites in close proximity.

Finally, I would like to thank USSTRATCOM and the ILRS for providing highprecision ephemerides on a variety of target spacecraft, for without those, this work would not have been possible. 


\section{Table of Contents}

Abstract $\quad$ iii

Acknowledgements $\quad$ iv

List of Tables $\quad$ X

List of Figures $\quad$ xi

List of Accronyms $\quad$ XV

Chapter 1 Introduction 1

1.1 Motivation . . . . . . . . . . . . . . . . 1

1.2 Problem Statement . . . . . . . . . . . . . . . . . 2

1.3 Previous Work . . . . . . . . . . . . . . . . . 3

1.3.1 Inertial-based Techniques . . . . . . . . . . . 3

1.3.2 LVLH-based Techniques ................ 4

1.4 Thesis Objectives . . . . . . . . . . . . . . . . . 6

1.5 Contributions . . . . . . . . . . . . . . . . 7

1.6 Organization ........................ 7

Chapter 2 Fundamental Concepts 9

2.1 Introduction . . . . . . . . . . . . . . . . . . . . 9

2.2 Satellite Orbits . . . . . . . . . . . . . . . 9

2.2.1 Orbit Classes ..................... 12

2.2.2 Reference Frames . . . . . . . . . . . . . . . . . . . 13

2.3 Space Surveillance . . . . . . . . . . . . . . . . . . . . . . . . 14

2.3.1 Ground-Based Observers . . . . . . . . . . . . . 14

2.3.2 Space-Based Observers . . . . . . . . . . . . 15

2.3.3 NEOSSat ........................... 16 
2.4 Relative Motion Dynamics . . . . . . . . . . . . . . . . . . . . 21

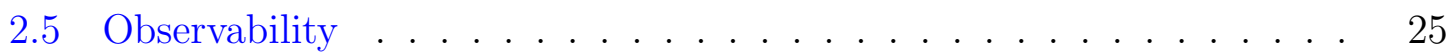

2.6 Relative Orbit Determination . . . . . . . . . . . . . . 28

2.6.1 Relative Orbit Observability . . . . . . . . . . . . . . . 29

2.6.2 Relative Orbit Observability Solutions . . . . . . . . . . 31

$\begin{array}{lll}\text { Chapter } 3 & \text { Proposed Methodologies } & 36\end{array}$

3.1 Introduction . . . . . . . . . . . . . . . . . . 36

3.2 Orbit Determination . . . . . . . . . . . . . . . 36

3.2 .1 Dynamics . . . . . . . . . . . . . . . . . . . . . 38

3.2 .2 Initial Orbit Determination $\ldots \ldots \ldots . \ldots \ldots$

3.2 .3 Least Squares . . . . . . . . . . . . . . . . . . . . . . 40

3.2 .4 Linearization . . . . . . . . . . . . . . . . . . . . 42

3.2.5 Differential Correction . . . . . . . . . . . . . . . . 43

3.2 .6 Covariance Matrices . . . . . . . . . . . . . . . . 45

3.2 .7 Sequential Processing . . . . . . . . . . . . . . 45

3.3 Method $1 \ldots \ldots \ldots \ldots \ldots \ldots \ldots \ldots \ldots$

3.4 Method $2 \ldots \ldots \ldots \ldots \ldots \ldots$

3.5 Metric Assessment . . . . . . . . . . . . . . . . . . . . . 48

$\begin{array}{lll}\text { Chapter } 4 & \text { Experiment Setup } & 51\end{array}$

4.1 Introduction . . . . . . . . . . . . . . . . 51

4.2 Close Approaches . . . . . . . . . . . . . . . . . . . . . 51

4.3 Observed Spacecraft $\ldots \ldots \ldots \ldots \ldots \ldots \ldots$

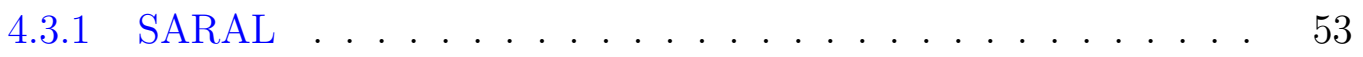

$4.3 .2 \quad$ Sapphire . . . . . . . . . . . . . . . . . 54

4.3 .3 BriteAustria . . . . . . . . . . . . . . . . . 54

4.3.4 UniBRITE-1 . . . . . . . . . . . . . . . . . . . . 55

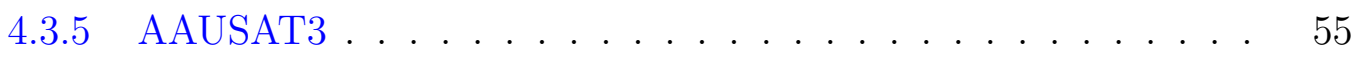

4.3 .6 QuikScat. . . . . . . . . . . . . . 56

4.4 Observation Events . . . . . . . . . . . . . . . . . 57 
4.4.1 Observation Schedule . . . . . . . . . . . . . 59

4.5 Data Processing . . . . . . . . . . . . . . . . . 59

4.5.1 NEOSSat Tasking . . . . . . . . . . . . . 60

4.5.2 Image Processing . . . . . . . . . . . . . . . . . . . 61

4.5.3 Reference Ephemerides . . . . . . . . . . . . . . . . 63

4.5.4 ODTK Orbit Determination . . . . . . . . . . . . 65

4.5.5 Generated Orbits vs. Reference Ephemerides . . . . . . . . . . 67

4.5.6 LVLH Transformation \& Exploitation . . . . . . . . . . . . . 67

4.6 Error Characterization . . . . . . . . . . . . . . 67

4.6.1 Data Source Errors . . . . . . . . . . . . . . . . . . 68

4.6.2 Observation Errors . . . . . . . . . . . . . . 68

4.6.3 Data Processing Errors . . . . . . . . . . . . . . . 69

$\begin{array}{lll}\text { Chapter } 5 & \text { Experiment Results } & 70\end{array}$

5.1 Introduction . . . . . . . . . . . . . . . . . . 70

5.2 Metric Assessment . . . . . . . . . . . . . . . . . 70

5.3 BriteAustria (1) . . . . . . . . . . . . . . 75

5.3 .1 Method $1 \ldots \ldots . \ldots . \ldots 75$

5.3 .2 Method $2 \ldots \ldots 76$

$5.3 .3 \mathrm{LVLH} \ldots \ldots . \ldots . \ldots . \ldots . \ldots 78$

5.4 UniBRITE-1. . . . . . . . . . . . . . . . . . . 81

5.4 .1 Method $1 \ldots \ldots . \ldots . \ldots . \ldots 81$

5.4 .2 Method $2 \ldots \ldots . \ldots . \ldots . \ldots 2$

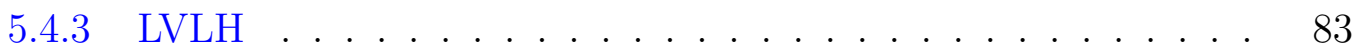

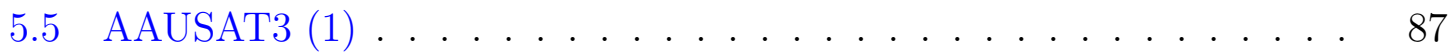

5.5.1 Method $1 \ldots \ldots . \ldots . \ldots . \ldots . \ldots 87$

5.5 .2 Method $2 \ldots \ldots \ldots . \ldots \ldots$

5.5.3 LVLH ............................ 90

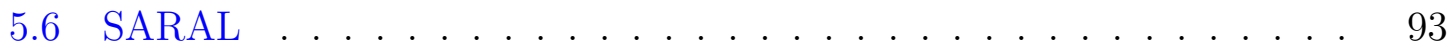

5.6 .1 Method $1 \ldots \ldots . \ldots . \ldots . \ldots 93$ 
5.6 .2 Method $2 \ldots \ldots \ldots \ldots$. . . . . . . . . . . . 94

$5.6 .3 \quad \mathrm{LVLH} \ldots \ldots \ldots \ldots \ldots \ldots$

5.7 AAUSAT3 $(2) \ldots \ldots \ldots \ldots \ldots \ldots$

5.7 .1 Method $1 \ldots \ldots \ldots$. . . . . . . . . . . . 99

5.7 .2 Method $2 \ldots \ldots \ldots \ldots$

$5.7 .3 \quad \mathrm{LVLH} \ldots \ldots \ldots \ldots \ldots$

5.8 BriteAustria $(2) \ldots \ldots \ldots \ldots \ldots$

5.8 .1 Method $1 \ldots \ldots \ldots$. . . . . . . . . . . . . 101

5.8 .2 Method $2 \ldots \ldots \ldots \ldots \ldots$

$5.8 .3 \quad \mathrm{LVLH} \ldots \ldots \ldots \ldots \ldots$

5.9 QuikScat . . . . . . . . . . . . . . . . . . . . . . . . . . 103

5.9 .1 Method $1 \ldots \ldots \ldots$. . . . . . . . . . . . . 103

5.9 .2 Method $2 \ldots \ldots \ldots \ldots \ldots$

$5.9 .3 \quad$ LVLH . . . . . . . . . . . . . . . . . . 105

5.10 Sapphire . . . . . . . . . . . . . . . . . . . 108

5.10 .1 Method $1 \ldots \ldots \ldots$. . . . . . . . . . . . 108

5.10 .2 Method $2 \ldots \ldots \ldots \ldots \ldots$

$5.10 .3 \mathrm{LVLH} \ldots \ldots \ldots \ldots \ldots \ldots$

5.11 Discussion . . . . . . . . . . . . . . . . . . . . . . . 111

$\begin{array}{lll}\text { Chapter } 6 & \text { Conclusion } & 113\end{array}$

6.1 Introduction . . . . . . . . . . . . . . . . . 113

6.2 Objectives Review . . . . . . . . . . . . . . . . . 113

6.3 Summary of Findings . . . . . . . . . . . . . . . . . . . . . . . 114

6.4 Recommendations for Future Work . . . . . . . . . . . . . . . 117

6.5 A Final Note . . . . . . . . . . . . . . . . . . . . . . . 118

$\begin{array}{ll}\text { Bibliography } & 119\end{array}$

Appendix A Data Formats 122

A.1 ILRS Standard Product 3 Format Ephemeris . . . . . . . . . . . . . 122 
A.2 STK Ephemerides . . . . . . . . . . . . . . . . . . 123

Appendix B ODTK Settings 


\section{List of Tables}

2.1 GPS Campaign Metric Results (values in arcseconds) . . . . . . 21

$4.1 \quad$ PSLV C20 Spacecraft Orbit Parameters . . . . . . . . . 52

4.2 QuikScat Orbit Parameters . . . . . . . . . . 57

$4.3 \quad$ Observation Events . . . . . . . . . . . . 59

$5.1 \quad$ Metric Assessment Results (values in arcseconds) . . . . . . . . 72

5.2 UniBRITE-1 Observations . . . . . . . . . . . 81

5.3 AAUSAT3 Observations . . . . . . . . . . . . . 87

$5.4 \quad$ Saral Observations . . . . . . . . . . . . . . 93

5.5 BriteAustria Observations . . . . . . . . . . . 101

5.6 QuikScat Observations .............. 103

$5.7 \quad$ Sapphire Observations . . . . . . . . . . . . . . 108

$5.8 \quad$ Results Summary . . . . . . . . . . . . . . . 112

B.1 ODTK Settings . . . . . . . . . . . . 124 


\section{List of Figures}

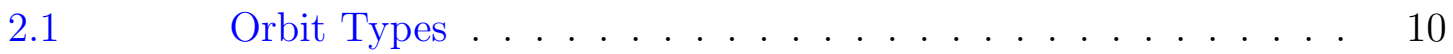

$2.2 \quad$ Keplerian Elements . . . . . . . . . . . . . . . . . 11

$2.3 \quad$ Orbit Classes $\ldots \ldots \ldots \ldots \ldots \ldots \ldots$

$2.4 \quad$ Artist's Rendition of NEOSSat . . . . . . . . . . . 17

$2.5 \quad$ NEOSSat TRM Image $\ldots \ldots \ldots \ldots \ldots$

$2.6 \quad$ NEOSSat SSM Image . . . . . . . . . . . . . . . . . 18

2.7 NEOSSat Science and Star Tracker CCD FOVs observing a GEO satellite . . . . . . . . . . . . . . . . 20

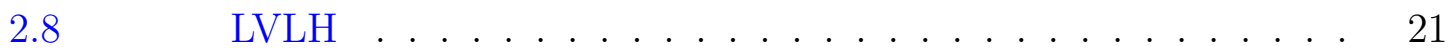

2.9 Target (red) and Chaser (blue) orbit planes intersecting . . . . 29

2.10 Shape Parameter [1] (modified) . . . . . . . . . . 30

2.11 LOS altered by manoeuvres [1] (modified) . . . . . . . . 32

2.12 Constrained Cylindrical Coordinates [2] (modified) . . . . . 34

$3.1 \quad$ Right Ascension and Declination $\ldots \ldots \ldots \ldots$

3.2 Initial Gooding Iteration . . . . . . . . . . . . 40

$3.3 \quad$ Final Gooding Iteration $\ldots \ldots \ldots \ldots$

$3.4 \quad$ Method 1 Data flow . . . . . . . . . . . . 47

$3.5 \quad$ Method 2 Data flow . . . . . . . . . . . . 48

4.1 Sapphire and NEOSSat aboard the PSLV dual launch adapter 52

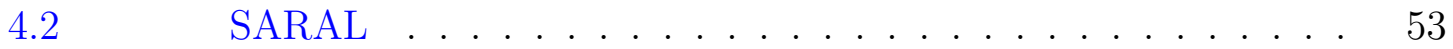

$4.3 \quad$ Artist's Rendition of Sapphire . . . . . . . . . . 54

$4.4 \quad$ BriteAustria and UniBRITE . . . . . . . . . . 55

$4.5 \quad$ AAUSAT3 $\ldots \ldots \ldots \ldots \ldots \ldots \ldots \ldots$

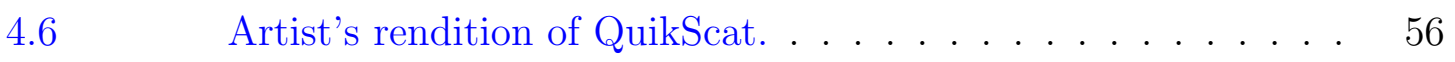

$4.7 \quad$ Close Approaches with NEOSSat . . . . . . . . 58

$4.8 \quad$ Data Flow for observation campaign $\ldots \ldots \ldots \ldots$ 
4.9 SARAL's Relative Angular Velocity relative to NEOSSat during an observation interval in $\operatorname{arcsec} / \mathrm{sec} \ldots \ldots . . . \ldots 61$

4.10 Predicted NEOSSat Orbit while observing SARAL on 8 Sep 2017. 62

4.11 Predicted NEOSSat observing intervals during the SARAL event on 7 and 8 Sep 2017. . . . . . . . . . . . . . . 63

$4.12 \quad$ NEOSSat Ephemerides compared to available TLE . . . . . 64

4.13 SARAL Ephemerides compared to available TLE $\ldots \ldots .65$

$5.1 \quad$ Right Ascension Residuals . . . . . . . . . . 71

$5.2 \quad$ Declination Residuals . . . . . . . . . . . . . . . 71

$5.3 \quad$ Tight RSO Signal . . . . . . . . . . . . . . . . 73

$5.4 \quad$ Saturated RSO Signal . . . . . . . . . . . . . 73

$5.5 \quad$ BriteAustria observation intervals from NEOSSat . . . . . 75

5.6 BriteAustria Opportunity \#1 Method 1 RIC Comparison . . . 76

5.7 BriteAustria Opportunity \#1 Method 2, 3- $\sigma$ filter RIC Comparison . . . . . . . . . . . . . . . . . 77

5.8 BriteAustria Opportunity \#1 Method 2, 5- $\sigma$ filter RIC Comparison . . . . . . . . . . . . . . . . . 77

5.9 BriteAustria Opportunity \#1 Method 2, Orbit Estimates . . 78

$5.10 \quad$ BriteAustria Opportunity \#1 LVLH . . . . . . . . . 79

$5.11 \quad$ BriteAustria Opportunity \#1 Orbit Estimate Errors . . . . . 80

5.12 UniBRITE-1 observation intervals from NEOSSat . . . . . 81

5.13 UniBRITE-1 Opportunity \#1 Method 1 RIC Comparison . . . 82

5.14 UniBRITE-1 Opportunity \#1 Method 2, 3- $\sigma$ filter RIC Comparison . . . . . . . . . . . . . . . . . 83

5.15 UniBRITE-1 Opportunity \#1 Method 2, 5- $\sigma$ filter RIC Comparison . . . . . . . . . . . . . . . . . 83

5.16 UniBRITE-1 Opportunity \#1 Method 2, Orbit Estimates . . . 84

5.17 UniBRITE-1 Opportunity \#1 LVLH . . . . . . . . 85

5.18 UniBRITE-1 Opportunity \#1 Orbit Estimate Errors . . . . 86 
$5.19 \quad$ AAUSAT3 observation intervals from NEOSSat . . . . . . 87

5.20 AAUSAT3 Opportunity \#1 Method 1, RIC Comparison . . . 88

5.21 AAUSAT3 Opportunity \#1 Method 2, 3- $\sigma$ filter RIC Comparison 89

5.22 AAUSAT3 Opportunity \#1 Method 2, 4- $\sigma$ filter RIC Comparison 89

5.23 AAUSAT3 Opportunity \#1 LVLH . . . . . . . . . . . . . . . . 91

5.24 AAUSAT3 Opportunity \#1 Orbit Estimate Errors . . . . . . . 92

5.25 SARAL observation intervals from NEOSSat . . . . . . . . . 93

5.26 SARAL Opportunity \#1 Method 1 RIC Comparison . . . . . 94

5.27 SARAL Opportunity \#1 Method 2, 3- $\sigma$ filter RIC Comparison 95

5.28 SARAL Opportunity \#1 Method 2, 5- $\sigma$ filter RIC Comparison 95

5.29 SARAL Opportunity \#1 LVLH . . . . . . . . . . . . . 97

$5.30 \quad$ SARAL Opportunity \#1 Orbit Estimate Errors . . . . . . . 98

$5.31 \quad$ AAUSAT3 observation intervals from NEOSSat . . . . . . . 99

5.32 AAUSAT3 Opportunity \#2 Method 2, 3- $\sigma$ filter RIC Comparison 100

$5.33 \quad$ BriteAustria observation intervals from NEOSSat . . . . . . 101

5.34 BriteAustria Opportunity \#2 Method 2, 3- $\sigma$ filter RIC Com-

parison .......................... 102

5.35 QuikScat observation intervals from NEOSSat . . . . . . 103

5.36 QuikScat Opportunity \#1 Method 1 RIC Comparison . . . . . 104

5.37 QuikScat Opportunity \#1 Method 2, 3- $\sigma$ filter RIC Comparison 105

5.38 QuikScat Opportunity \#1 LVLH . . . . . . . . . 106

5.39 QuikScat Opportunity \#1 Orbit Estimate Errors . . . . . . 107

$5.40 \quad$ Sapphire observation intervals from NEOSSat . . . . . . . 108

5.41 Saturated CCD Image of Sapphire as detected by NEOSSat at a separation of $32 \mathrm{~km}$. . . . . . . . . . . . . . 109

5.42 Saturated CCD Pixels of Sapphire as detected by NEOSSat at a separation of $32 \mathrm{~km}$. . . . . . . . . . . . . 109

5.43 Sapphire Opportunity Method 2, 3- $\sigma$ filter RIC Comparison 110 
A.1 SARAL Standard Product 3 Format ephemeris from the Centre National d'Études Spatiales . . . . . . . . . . . . . . 122

A.2 SARAL Ephemeris in STK Format . . . . . . . . 123 


\section{List of Accronyms}

$\begin{array}{ll}\text { 18SPCS } & \text { 18 Space Control Squadron } \\ \text { AGI } & \text { Analytical Graphics Inc. } \\ \text { AIS } & \text { Automated Identification System } \\ \text { BRITE } & \text { Bright Target Explorer } \\ \text { CCD } & \text { Charged Coupled Device } \\ \text { CSA } & \text { Canadian Space Agency } \\ \text { DC } & \text { Differential Correction } \\ \text { DEC } & \text { Declination } \\ \text { DND } & \text { Department of National Defence } \\ \text { DRDC } & \text { Defence Research and Development Canada } \\ \text { ECEF } & \text { Earth-Centered-Earth-Fixed } \\ \text { ECI } & \text { Earth-Centered-Inertial } \\ \text { EKF } & \text { Extended Kalman Filter } \\ \text { FOV } & \text { Field of View } \\ \text { GEO } & \text { Geostationary Earth Orbit } \\ \text { GPS } & \text { Global Positioning System } \\ \text { HEO } & \text { Highly-Elliptical Orbit } \\ \text { HEOSS } & \text { High Earth Orbit Space Surveillance } \\ \text { IIOD } & \text { Initial Inertial Orbit Determination } \\ \text { ILRS } & \text { International Laser Ranging Service } \\ \text { IOD } & \text { Initial Orbit Determination } \\ \text { IROD } & \text { Initial Relative Orbit Determination } \\ \text { ISS } & \text { International Space Station } \\ \text { J2000 } & \text { J2000 Reference Frame } \\ \text { KF } & \text { Low Earth Orbit } \\ \text { LEO } & \text { Lincoln Labs } \\ \text { LL } & \end{array}$


LOS

LS

LVLH

MDA

MEO

MIT

MOST

NASA

NCC

NEOSSat

NESS

ODTK

OD

OOD

PSLV

RA

RAAN

RIC

RMS

RSO

SAA

SGP4

SBSS

SBV

SFL

SP

SSC

SSM

SSN

STK
Line-of-Sight

Least Squares

Local-Vertical-Local-Horizontal

MacDonald Dettweiler and Associates

Medium Earth Orbit

Massachusetts Institute of Technology

Microvariability and Oscillations of STars

National Aeronautics and Space Administration

NEOSSat Constraint Checker

Near Earth Object Surveillance Satellite

Near Earth Space Surveillance

Orbit Determination Tool Kit

Orbit Determination

Optimal Orbit Determination

Polar Satellite Launch Vehicle

Right Ascension

Right Ascension of the Ascending Node

Radial, In-track, Cross-track

Root Mean Square

Resident Space Object

South Atlantic Anomaly

Simplified General Perturbations-4

Space Based Space Surveillance

Space Based Visible

Space Flight Laboratory

Special Perturbation

SciSat Command

Star Stare Mode

Space Surveillance Network

Systems Tool Kit 
SQUID3

USAF

USSTRATCOM

UTC

TLE

TRM
Semi-QUIck Detection 3rd Iteration

United States Air Force

United States Strategic Command

Universal Time Coordinated

Two Line Element

Track Rate Mode 


\section{Chapter 1}

\section{Introduction}

\subsection{Motivation}

Since the advent of the space age, an ever increasing number of artificial satellites have been launched. In the early days of space flight, the high cost of placing satellites in orbit led to the field being primarily dominated by governments. However, due to advancements of various technologies over the past decade, the stage has been set for what promises to be an exciting and challenging decade for commercial space operations and experimentation.

Ever since the 1960's Space Race, proximity operations has been a studied problem. Finding it's genesis in Project Gemini to dock spacecraft together as an enabler to reach the Moon, the application of proximity and rendezvous operations is applicable even more so today with famous examples such as the building and support of the International Space Station (ISS). Commercial and academic institutions have begun to carry out proximity operation experiments with increasingly smaller objects [3]. Commercial advancements in the miniturization of space components, as well as the increasing affordability of space launch opportunities is proving to be a watershed moment in history with the impending explosion in the numbers of satellites on orbit.

Accompanying the growth of objects in space was both the need and capability to track them. One of the most well-known space tracking organizations in the world is the United States Air Force (USAF). The USAF tracks space objects using the Space Surveillance Network (SSN) by means of ground-based and space-based sensors [4]. Sensors in the SSN are designed to perform surveillance on different orbit classes based upon their inherent operational strengths. Generally, all objects surveilled by these sensors are hundreds to thousands of kilometers away, distances that are one or more orders of magnitude larger than the distance associated with proximity operations. 
Although never intended to carry out this purpose, space based sensors can in theory be re-purposed to perform orbit determination on objects in close proximity [5]. Investigating the feasibility and challenges of re-purposing a space surveillance platform, originally designed to surveil distant objects, for the application of determining relative positions between spacecraft undergoing proximity operations and events is the primary motivation of this work.

\subsection{Problem Statement}

Optical systems are the most commonly used space surveillance platforms for deep space object tracking primarily due to their lower operating cost. Unfortunately, the downside to optical systems is their inability to directly measure distance to an object and as such, the observations collected by these systems are called anglesonly measurements. One of the primary issues surrounding proximity operations using angles-only measurements is that inability to directly measure range to an object. This complicates proximity operations as there is a requirement for one of the spacecraft, the chaser, to uniquely determine its position with respect to the other spacecraft, the target, for guidance purposes. This process of determining the relative orbit of the target spacecraft is particularly challenging due to the formulation of the relative reference frame that proximity operations are described in. This reference frame is generally referred to as the Local-Vertical-Local-Horizontal (LVLH). Within this reference frame, the location of the measuring instrument is in close proximity to the origin of the reference frame. This close proximity provides an insufficient offset between measuring instrument and origin, resulting in an inability to uniquely identify the relative orbit using optical measurements in its present form. Despite this downfall for angles-only measurments, the LVLH reference frame is a perfectly valid and arguably the best reference frame to work in due to its relative simplicity and effectiveness to describe the motions of one object in proximity to another.

Space surveillance operations within the SSN utilize a global collection of sensors to track space objects and use an alternative reference frame to track objects. The network works in Earth-centered reference frames such as Earth-Centered-Earth-Fixed (ECEF) or Earth-Centered-Inertial (ECI). Just like LVLH, these reference frames 
are chosen for the intended task of describing the motions of objects with respect to a position on the Earth. Sensors feeding a surveillance system such as the SSN work in these inertial frames and allow the SSN to determine the inertial orbit of an object about the Earth. Unfortunately, the process of orbit determination in the inertial frame has no simple closed-form solution such as that in the relative LVLH, and requires the orbits to be determined with an iterative approach, increasing both complexity and computational requirements.

This thesis is concerned with investigating the feasibility and challenges associated with using existing space-based surveillance platforms operating in an inertial reference frame performing inertial orbit determination and subsequently transcribing it to the relative LVLH reference frame as a solution to the relative orbit determination problem for angles-only measurements.

\subsection{Previous Work}

This section presents previous work completed on the topic of orbit determination for both inertial and relative reference frames, some associated challenges, as well as some potential solutions.

\subsubsection{Inertial-based Techniques}

The inertial orbit determination problem is an old one, dating back to the Hellenic period with early astronomers studying the motion of the stars and planets. The process of orbit determination arguably took off with Yohannes Kepler's work to predict for the movement of Mercury in his 1627 work titled Rudolphine Tables [6]. This work was built upon by various authors such as Isaac Newton in his 1687 work titled Philosophiæ Naturalis Principia Mathematica which introduced the first practical method to determine the orbit of an object from three observations [6].

The work carried out by Johann Lambert is one of the most influential due to his solution which enabled the finding of both elliptical and hyperbolic orbits in addition to circular ones. His solution allowed for the taking of two known positions of an object separated by a time interval and subsequently fully determining the orbit of that object. This solution is now known as Lambert's Problem, and is the fundamental 
basis for many modern orbit determination solutions [6].

Pierre-Simon Laplace developed the first orbit determination technique based on angles-only information in 1780. This method was in contrast to others already developed which required knowledge, or at least a guess, at the range of the object in each observation. This was a particular challenge as the only observation systems available at the time were the inherently range-less optical telescope systems. Unfortunately Laplace's techniques are quite intensive and are only made practical when computers are used to process the numerous calculations [6].

Carl Freidrich Gauss, already a great mathematician, proved his merit as an astronomer when he successfully predicted the orbit of the minor planet Ceres in 1801, allowing for astronomers to re-locate it at the end of the year. This event marked the first practical initial orbit determination process. His method involved using the Right Ascension and Declination of three observations of the planet in order to determine the orbit [6].

This collection of early work has become the foundation for modern orbit determination techniques. While the majority of early work focussed on deterministic methods to solve orbits with the same number of unknowns as available observations, modern methods have shifted their focus to employ statistical techniques due to the proliferation of available observations [7]. By linearizing the non-linear problem, more complicated force models incorporating drag and solar radiation pressure can be used for better orbit estimation [8].

\subsubsection{LVLH-based Techniques}

Similar to inertial orbit determination, there has been significant work on the topic of relative orbit determination.

Since the 1960's Apollo program, the problem of rendezvous and proximity operations has been well studied [9]. With the increasing availability of microsatellite platforms in recent years, extensive work has been performed to model proximity operations dynamics between increasingly smaller spacecraft $[3,10]$. As opportunities for space operations by the commercial and academic community increases, especially for those in the field of relative navigation, it is logical to assume that study on the 
topic will continue to grow in support of those future missions.

One interesting field of study within rendezvous and proximity operations is the use of onboard cameras for relative navigation purposes. The use of these cameras allow for the spacecraft to literally see where it is with respect to another object and provides a simple, low cost, effective relative navigation sensor. Despite the substantial leap forward that this presents, there is one fundamental flaw in solely using an angles-only camera for proximity operations. Being unable to directly determine range to the spacecraft results in numerous possible solutions when attempting to solve for the relative orbit of the other spacecraft [11].

This problem is the mathematical issue of observability; not to be confused with object detectability. The problem of observability is present in any reference frame, but can be particularly challenging for LVLH operations. In using angles-only measurements from the spacecraft in the LVLH reference frame, observations are made from a camera close to the origin of the chaser's reference frame. This co-location results in an insufficient parallax, defined as the difference in apparent position of an object due to the physical separation of the observer from two observation points. This insufficient difference in position from the two observing locations prevents the ability to infer range to the spacecraft and therefore be unable to uniquely estimate the relative state of that spacecraft [11].

This issue of observability for proximity operations can be overcome by implementing numerous practical solutions to the problem. If an observing spacecraft is capable of propulsion, the use of specifically calibrated manoeuvres can be used to alter the line of sight to the object [1]. However, this solution has a significant downside as it requires the expenditure of fuel, a valuable and limited resource on a satellite. It is possible to utilize prior knowledge of the size and shape of an object in order to infer distance by comparing the angular size of the object and using trigonometry to determine the range $[12,13]$. Unfortunately, except for specifically designed missions, it may not be possible to obtain the a-priori information required regarding another satellite's design, or even more applicable, the shape and size of uncharacterized debris. Promising theoretical work includes off-setting the camera from the center of mass of the chaser spacecraft in order to create sufficient parallax $[12,13]$. 
This solution is partially practical for large spacecraft operating in close proximity of $<100 \mathrm{~m}$ due to the larger offsets which could be utilized by the physical size of the spacecraft, however, it is impractical for smaller satellites such as microsatellites and nanosatellites. The use of a specially designed and constrained cylindrical coordinate frame is possible, where the orbit's angular information is theorized to be better preserved due to the nature of the reference frame [2]. However, this may not be the most effective solution as there are several practical limitations on observation timing in order to obtain a sufficiently accurate orbit estimate. It is possible to utilize a second camera system to implement stereo vision to determine range to the object, but again this solution may bring about similar problems associated with the size of the camera offsets [13].

All of this previous work approaches the issue of solving for the relative state of the other object within the LVLH frame. Some of this work acknowledges or at least alludes to, that although more difficult, implementing inertial orbit determination solutions is possible $[1,5]$. This process would involve first solving for the inertial orbit of the observed spacecraft, and then projecting that solution into the LVLH reference frame to be exploited.

\subsection{Thesis Objectives}

The objective of this thesis is to investigate the feasibility and challenges associated in re-purposing a space-based surveillance platform designed for inertial orbit determination, the NEOSSat microsatellite, to determine the relative orbit of a target spacecraft. In support of this objective, several sub-objectives were identified:

- Confirm if NEOSSat is able to successfully acquire sufficient observation data from an object in proximity $(<2000 \mathrm{~km}$ range)

- Determine if the acquired observation data contains sufficient meaningful information required to generate an inertial orbit

- Determine if there is an optimal observation time-spacing to generate a relative orbit for an object in proximity to NEOSSat 
- Determine if the calculated inertial orbit can be transformed to the relative reference frame for exploitation

- Assess the accuracy of the resulting inertial orbit against high-precision orbit ephemerides

- Assess the feasibility of implementing this methodology on future space surveillance spacecraft

\subsection{Contributions}

The contributions of this work to the field of space surveillance and spacecraft formation flight are:

- An investigation on the feasibility of using inertial orbit determination techniques for spacecraft formation flight

- Exploration of the challenges for the implementation of EKFs on LEO to LEO observations using an optical observer

- An initial characterisation of LEO to LEO optical observation noise for NEOSSat in comparison to LEO to MEO

- An initial estimate at the range threshold in which optical LEO to LEO space surveillance operations for NEOSSat are no longer viable

\subsection{Organization}

The remainder of the work outlined in this thesis is organized as follows:

Chapter 2: Fundamental Concepts: Explores fundamental space surveillance concepts, relative motion dynamics, relative orbit determination, and the issue of observability.

Chapter 3: Methodology: Outlines the fundamentals of inertial orbit determination as well as the approach employed by the author to apply inertial orbit determination for applications in proximity and rendezvous operations. Additionally, the process for the metric assessment of the sensor in this configuration is outlined. 
Chapter 4: Experiment Setup: Outlines the series of satellites undergoing close approaches with NEOSSat, an outline of the events which NEOSSat observed the satellites, as well as the steps used to process data. The subsequent transformation to the LVLH reference frame for exploitation as well as the characterization of the metric accuracy of the sensor is also explored.

Chapter 5: Experiment Results: Covers and discusses the results from the methodologies outlined in Chapter 3 when applied to the events in Chapter 4.

Chapter 6: Conclusion: Ends this thesis with conclusions reached, contributions, as well as recommendations for future work. 


\section{Chapter 2}

\section{Fundamental Concepts}

\section{$2.1 \quad$ Introduction}

This chapter presents the fundamental basis of knowledge required to support the work undertaken in this thesis. A review of satellite orbits, space surveillance, observability, the orbit determination concept, relative motion dynamics, as well as relative orbit determination and the associated challenges will be covered.

\subsection{Satellite Orbits}

Newton described the trajectory of an object under the influence of a central acceleration and identified the $\frac{1}{r^{2}}$ range and mass dependence where $r$ is the distance between the object centers. Objects orbiting another in space move in a fashion that can be visualized as slices of a conic section, these orbits can either be open or closed. Open orbits describe one-time occurrences where an object enters the gravitational sphere of influence of another body and then transits out, never to return. These orbits are termed either parabolic or hyperbolic depending on a quantitative value called eccentricity. Closed orbits are bounded and an object continually circles the larger object in either a circular or eccentric orbit. These orbits can be visualized in Fig. 2.1 and are governed by Eq. (2.1) where $\vec{r}$ is the vector from the center of the larger object to the position of the smaller object, $\ddot{\vec{r}}$ is the acceleration along $\vec{r}$, and $\mu$ is the standard gravitational parameter of the larger object.

$$
\ddot{\vec{r}}=-\mu \frac{\vec{r}}{|r|^{3}}
$$

In order to describe the position and velocity of an object (ie. it's state) in threedimensional (3D) space, six values are required. The state of the object is described by its position and velocity at a specific point in time called the epoch. There are 

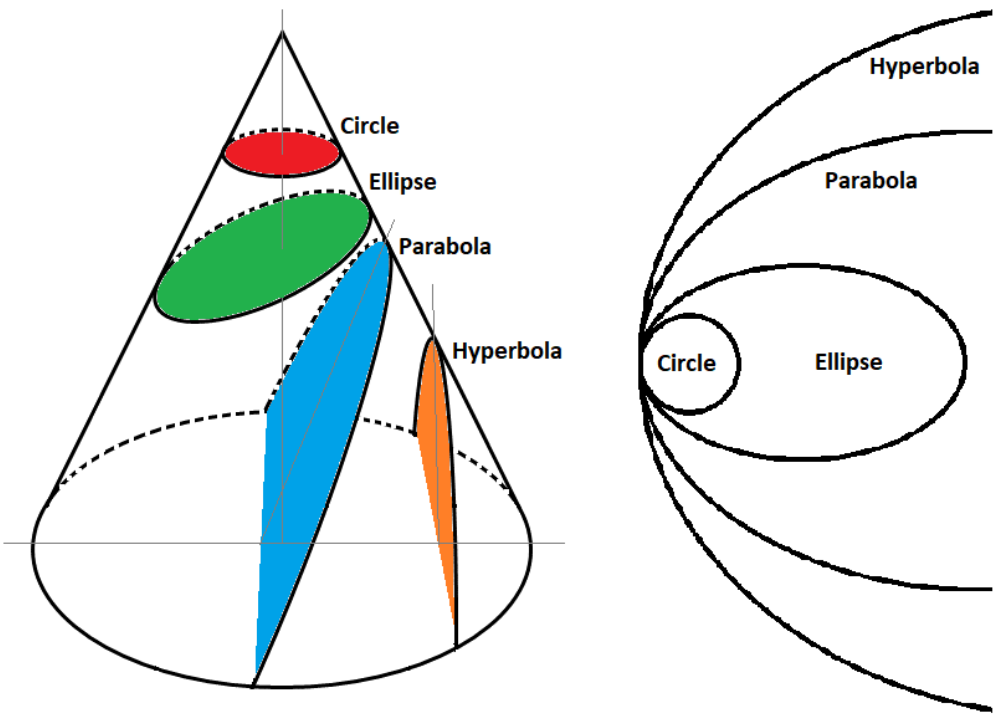

Figure 2.1: Orbit Types

multiple ways to describe the state of an object, the two most commonly used in space applications are the Classic Keplerian Elements, and Cartesian Coordinates. Keplerian elements comprise six values: semimajor axis $(a)$ determines the size, eccentricity (e) describes the shape, while inclination $(i)$, right ascension of the ascending node $(\Omega)$, and argument of perigee $(\omega)$ describe the orientation of the orbit in $3 \mathrm{D}$ space, and finally the true anomaly $(\nu)$ describes where the object is within the orbit at the prescribed epoch. Cartesian coordinates describe the position and velocity in 3D space with six elements: $[x y z \dot{x} \dot{y} \dot{z}]^{\top}$. Keplerian elements and Cartesian coordinates are both perfectly valid representations, and their use depends on both the application of the orbit, as well as the preference of the user. For example, it may be easier for a user to visualize the orbit using Keplerian elements, while Cartesian coordinates are generally preferred for computation. A user can convert from one representation to another by applying the appropriate methodology.

If the state of the object is known, the object's future state can be determined by propagating it forwards in time. In order to propagate the object, an appropriate force model is required. To the author's knowledge, there are currently no force models that perfectly describe the forces that act upon an object in space. Despite tremendous effort by many researchers, the complexity of orbit estimation involves a variety of 


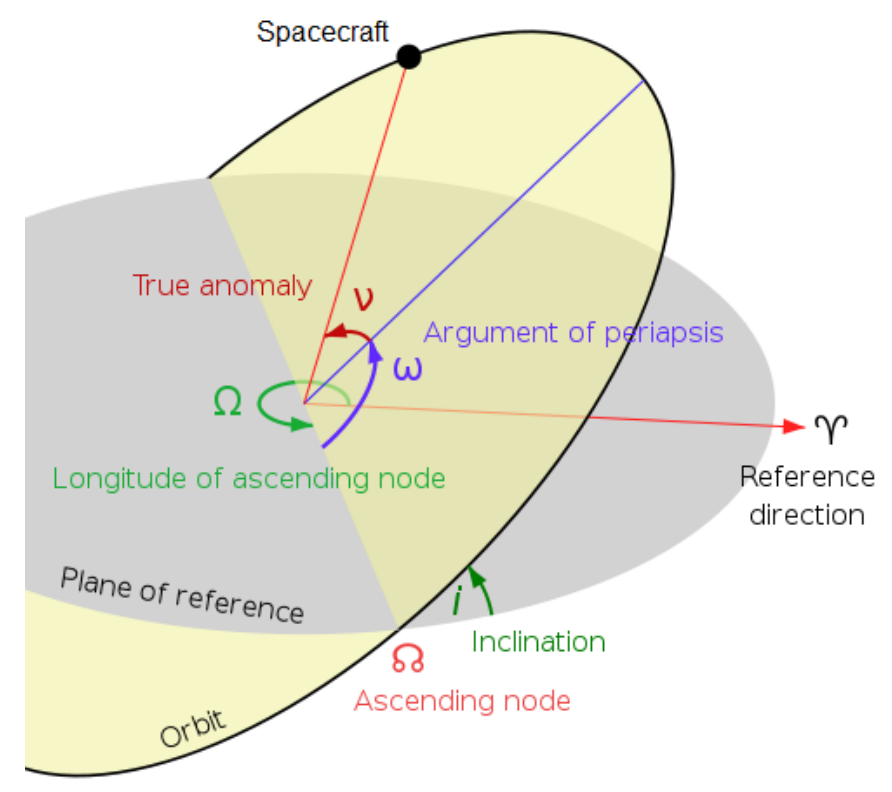

Figure 2.2: Keplerian Elements ${ }^{1}$ (modified)

factors not limited to: gravitational non-homogeneity of the Earth, gravitational influences of other celestial bodies, atmospheric drag, and solar radiation pressure. Despite this shortfall, there are several excellent propagators that exist which model the variety of influencing forces as closely as possible given system computational limits. With these propagators, it is important to differentiate between the two major types: analytical, and numerical. Analytical propagators utilize simplified equations to determine, based on the original object state, where the object will be after a specified time span. These propagators produce approximate, or general $[6,14]$ results, and are often characteristic of early efforts to model spaceflight on early computer systems. The most famous example is the Simplified General Perturbations-4 (SGP4) algorithm [15]. SGP4 is written to work with the standard Two-Line-Element set (TLE), a coarse approximation of an object's orbital elements which is sufficient for a large variety of purposes such as calculating overflight, and for antenna pointing. Unfortunately, TLEs, with their simplified exponential atmosphere drag model, are not the most accurate with position errors beginning at $1 \mathrm{~km}$ and growing at a rate of 1-3 km per day depending on altitude $[16,17]$.

\footnotetext{
${ }^{1}$ Keplerian Elements, 2007, (Accessed July 25, 2018), https://upload.wikimedia.org/wikipedia/commons/e/eb/Orbit1.svg
} 
Numerical propagators differ significantly from analytical methods where numerical propagators start with the initial state of the object and numerically integrate the equations of motion. Each step involves the process of integrating the modelled forces acting on the object over that time step and use the resulting value to update the object state, which is then repeated for each subsequent step. The size of these steps can vary, but are generally very small, requiring an order of magnitude jump in the number of calculations. The result is an orbit model that is significantly more accurate, but the increase in the number of calculations result in a substantial increase of computational power and a correspondingly longer time period in order to perform the processing [17]. This algorithm leads to a set of very specific, or special results $[6,14]$. The by-product of numerical propagators is a time-stamped series of points in 3D space called ephemerides. Generating these ephemerides is incredibly useful as they can then be loaded into other systems to provide high-accuracy position information without the requirement to re-perform time-consuming calculations. One set of high-precision ephemerides, formally called Special Pertubation (SP) ephemerides, are generated by the USAF's 18th Space Control Squadron (18SPCS).

\subsubsection{Orbit Classes}

Objects orbiting the Earth are sub-divided into orbit classes. In ascending order by distance from the Earth, there are Low Earth Orbits (LEO), Medium Earth Orbits (MEO), Geostationary Earth Orbits (GEO), as well as Highly-Elliptical Orbits (HEO), which themselves span a variety of distances. LEO orbits span from the upper limits of the Earth's atmosphere to an approximate altitude of 2,000 km, this region is mostly characterized by earth observing satellites, orbital debris such as expended rocket bodies, as well as the International Space Station (ISS). MEO orbits span from the lower LEO limit of 2,000 km to $35,786 \mathrm{~km}$, and is a mostly empty region except for a variety of positioning system satellites such as the Global Positioning System (GPS) orbiting around 20,000 km, as well as more orbital debris such as expended rocket bodies. GEO orbits occupy a unique altitude of $35,786 \mathrm{~km}$ which results in the orbital period matching that of the rotational period of the Earth, allowing for a variety of convenient operations such as satellite communications. HEO orbits cover 
a wide variety of altitudes and are occupied by a variety of satellites with unique missions exploiting the geometry of these orbits.

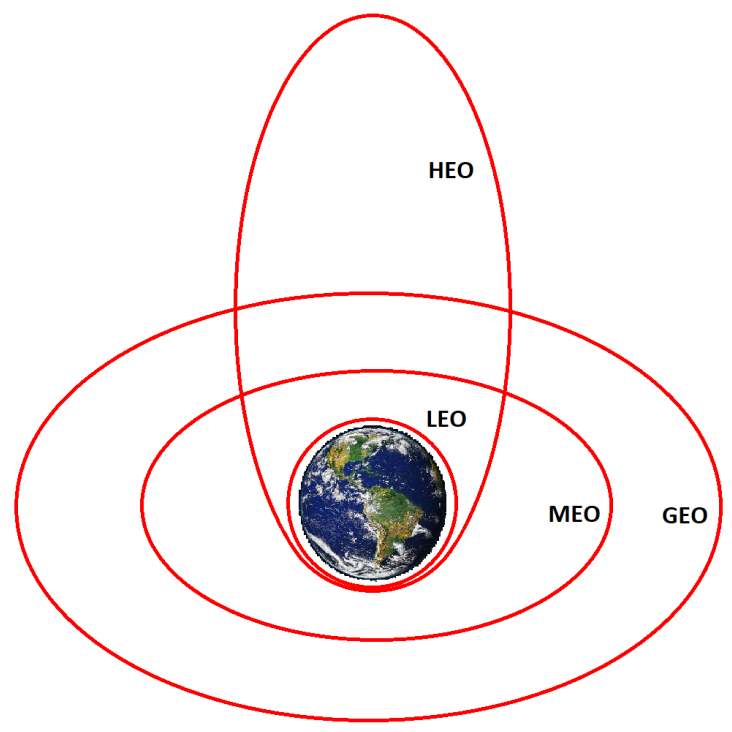

Figure 2.3: Orbit Classes

\subsubsection{Reference Frames}

Although previously discussed in Section 2.2 that satellite orbits are fully represented by means of the object's state in different representations such as Keplerian elements or Cartesian coordinates, it is important to consider the reference frame that the state is described in. In space, there is no such thing as an inertial reference frame due to the constant motion of objects in an expanding universe. There are, however, some reference frames which closely approximate an inertially fixed frame centered on the Earth. One of the standard inertial reference frames in use is J2000. Named for its epoch of 12:00:00 on January 1st, 2000, this system, as per USAF definition, aligns the $X$ axis towards the position of the Vernal Equinox, the $Z$ axis to the pole of the celestial sphere, with the $Y$ axis completing the right-handed reference frame. This reference frame is centered on the Earth and is fixed relative to the celestial sphere.

The use of different reference frames often depend on the intended audience of that information. Although useful for space applications, some reference frames are more appropriate for Earth-oriented purposes. A commonly used Earth-fixed reference 
frame is the Earth-Centered-Earth-Fixed (ECEF) frame which is fixed at the center of the Earth, but rotates with the rotation of the Earth. This reference frame is often used for locating the longitude and latitude of an object on the surface of the Earth. The $X$ axis points outwards aligned with the Equator from $0^{\circ}$ longitude, the $Z$ axis points up through the Earth's geographic North Pole, with the $Y$ axis completing the right-handed reference frame. In space operations applications, it is often used for GPS ephemerides or geodesy.

Separate from these two reference frames is what will be called the sensor reference frame. The sensor reference frame, like either J2000 or ECEF, may be more useful depending on the work being undertaken. For a ground-based observer looking at a satellite passing overhead, using a system of azimuth and elevation centered on the position of the telescope mount and oriented with respect to the Earth's surface would be more useful in order to steer the telescope to follow the object across the sky than the direct J2000 or ECEF position values. The use of a reference frame best suited for the intended objective can also be extended to space-based systems and will be expanded on in Section 2.4.

\subsection{Space Surveillance}

With the launch of Sputnik, the need to track artificial objects in orbit appeared. No longer was the emphasis of looking at space oriented on distant celestial objects, it was now rapidly becoming oriented on the monitoring of satellites. One of the largest efforts in this field was the development of the United States' SSN [4]. Originally comprised of optical systems, the network grew over time to include a variety of systems, with the most emphasis on optical and radar sites.

\subsubsection{Ground-Based Observers}

Optical systems are relatively simple in design. They are passive systems which concentrate sunlight reflected from a satellite onto a focal plane where a detector registers the detected photo electrons. These systems began as film-based cameras and were unable to directly measure range to an object; as such, they provided anglesonly measurements of the position of an object with respect to a coordinate frame. 
Early space surveillance examples of such systems were the Baker-Nunn cameras operated by Canada in support of the SSN [18]. With the advent of the Charged Coupled Device (CCD), electronic silicon detectors designed to detect photons, film cameras for space surveillance were gradually replaced as CCDs could offer superior accuracy and sensitivity due to their digital nature. CCDs count individual photons of light from a detected object, and when striking the individual cells of the CCD array, convert these photons into an electric charge which is then counted and associated to the specific detector pixel using a readout register.

Radar systems are able to accurately measure distance to an object in contrast to optical systems. Radars are active sensors which emit busts of radio energy and subsequently wait for a tiny return echo from the measured object. If a radar is able to measure the Doppler shift of a returning signal, they can estimate an object's range rate, adding more information to what can be collected in a single measurement. Radars are generally classified as mechanical or phased array; mechanical radars are large dishes that are mechanically steered to point in a direction while phased array radars are flat surfaces with an array of dipoles which electronically steer beams of RF energy into space. Due to their active nature, radar pulses attenuate as a function of the inverse of the square of the range to an object. Taking into account a pulse's return trajectory, radar signals are further attenuated making the return pulse's energy proportional to the inverse of the range raised to the fourth power. This primarily limits space surveillance radars in the SSN to operate in the LEO regime. Unfortunately, although capable of providing higher fidelity data, radars are much more expensive systems due to their power needs and are generally used by larger entities such as companies and governments.

\subsubsection{Space-Based Observers}

To the author's knowledge, there are only government-funded optical systems in orbit which are designed to perform space surveillance in support of space catalog maintenance. In the mid-1990s, the Massachusetts Institute of Technology (MIT)/Lincoln Labs (LL) undertook an effort to place an optical space surveillance platform in 
space in an effort to overcome some of the limitations on ground based optical systems such as weather, the day-night cycle, as well as to ensure survivability in future conflicts. These efforts resulted in the Space-Based Visible sensor (SBV) and, later, the Space Based Space Surveillance (SBSS) mission $[4,19]$. In the mid-2000s, the Canadian Department of National Defence (DND) undertook an effort to create the Sapphire microsatellite, essentially a scaled-down SBV in a microsatellite bus [20]. Around the same time, Defence Research and Development Canada (DRDC) with the Canadian Space Agency (CSA) undertook an effort to construct an R\&D space surveillance platform which eventually became the Near Earth Object Surveillance Satellite (NEOSSat) [21].

\subsubsection{NEOSSat}

NEOSSat is a dual mission space telescope that was launched on 25 February 2013 in a polar sun-synchronous LEO orbit and was designed to conduct a space surveillance mission as well as detect near-Earth asteroids. The two missions undertaken by NEOSSat are the High Earth Orbit Space Surveillance (HEOSS) mission and Near Earth Space Surveillance (NESS). The former is a Space Situational Awareness (SSA) mission focusing on the performance of metric observations on Resident Space Objects (RSOs) primarily in the deep space region. This region is defined by the USAF as objects with orbital periods greater than 225 minutes, including MEO, HEO, and GEO orbit classes. The latter mission was focused on tracking near-Earth asteroids in a heliocentric orbit within the radius of Earths orbit, at low solar elongations [21].

NEOSSat balanced the two main missions by allocating different calendar days to each mission. For the NESS mission, the satellite would point in a solar direction, up to $45^{\circ}$ off the Sun, while the HEOSS mission looked in what was generally an anti-solar direction, with occasional observations on objects with solar phase angles greater than $90^{\circ}$. These drastically different payload operating areas resulted in a complex set of design requirements, ultimately resulting in the final design.

NEOSSat had a launch mass of $74 \mathrm{~kg}$ and is approximately $1.4 \times 0.8 \times 0.4 \mathrm{~m}$ in size. The satellite was built on the same bus architecture as the MOST microsatellite [22], with some structural adjustments to accommodate for the different payload and 


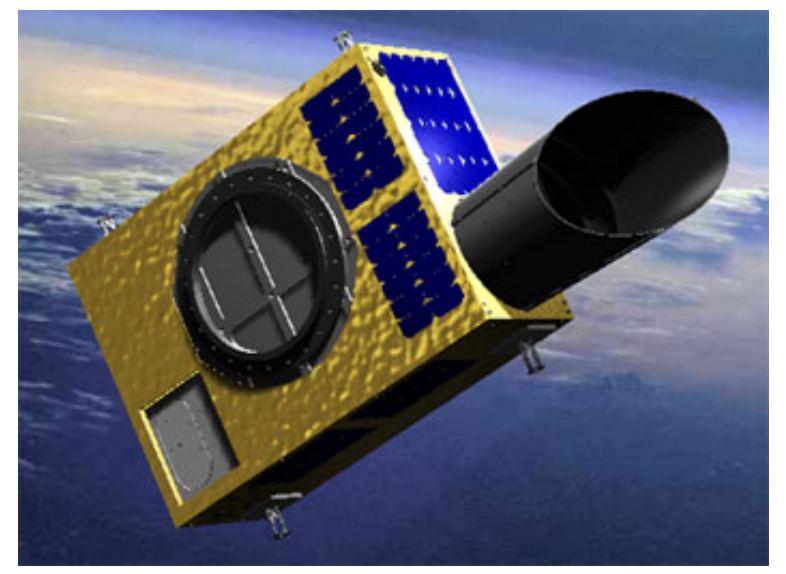

Figure 2.4: Artist's Rendition of NEOSSat ${ }^{2}$

operating modes. A polar sun-synchronous orbit altitude of $786 \mathrm{~km}$ was selected for the satellite, which proved to be an ideal configuration as the GEO belt is continuously viewable for the entire orbit due to the relation between its orbit and that of the GEO belt. All GEO objects are, in theory, viewable by the satellite over a 24 hour period, with practical operation constraints for the satellite including: overflight of the South Atlantic Anomaly (SAA), a minimum Earth-limb exclusion, as well as exclusion cones about bright objects such as the Moon and Sun [17,22]. In addition to these exclusion zones, NEOSSat is constrained by the angular rate of the targets it can track. With the science instrument being body-fixed on the satellite, it must utilize its four reaction wheels to rotate the satellite towards a target in order to observe it. In its imaging mode, NEOSSat can take either Track Rate Mode (TRM) seen in Fig. 2.5, where NEOSSat follows an object and the stars appear as streaks, or Star Stare Mode (SSM) images seen in Fig. 2.6 where NEOSSat stares at a particular portion of the celestial sphere and the background stars appear circular while satellites appear as streaks.

NEOSSat's payload is a $15 \mathrm{~cm}$ diameter Maksutov Cassegrain optical telescope equipped with a specially designed baffle to minimize stray light reflecting along the tube to the detecting CCD surfaces. The satellite has two identical CCDs co-located on the focal plane, one used as a star tracker to provide the necessary attitude control

\footnotetext{
${ }^{2}$ MSCI, NEOSSat SPECS, 2013, (Accessed June 28, 2018), http://www.mscinc.ca/products/NEOSSat.html
} 


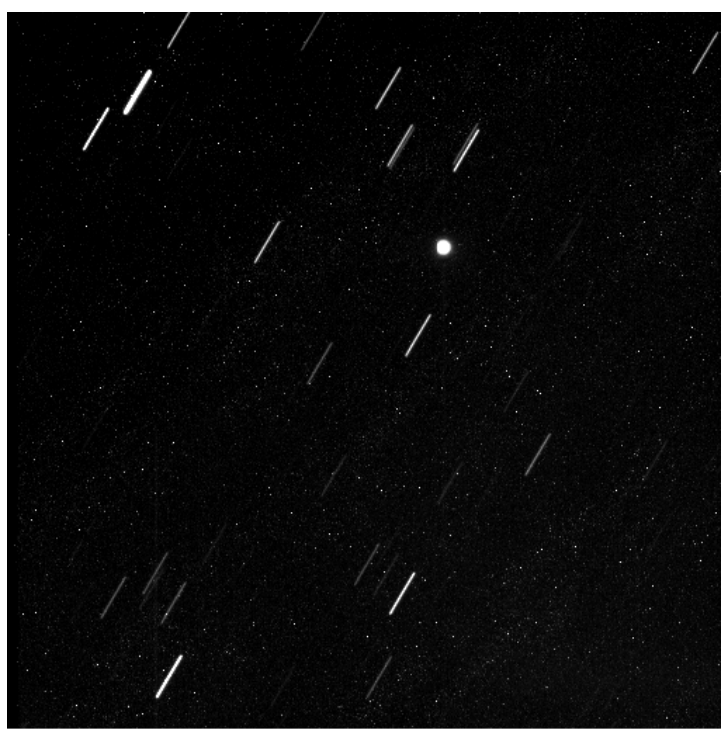

Figure 2.5: NEOSSat TRM Image

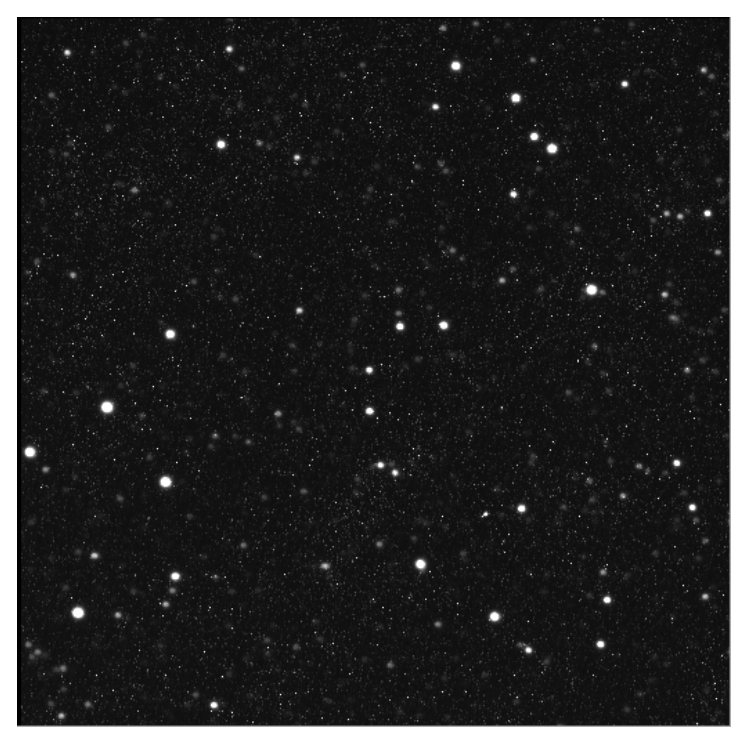

Figure 2.6: NEOSSat SSM Image

system sensor input, and the other is the main science instrument. The onboard CCDs each have an array of $1056 \times 1030$ square pixels, with each pixel measuring $13.3 \mu \mathrm{m}$. This pixel size, along with the focal ratio of $f / 6$, gives each pixel a three arcsecond scale and a resulting instantaneous Field of View (FOV) of $0.88 \times 0.86$ degrees. NEOSSat operates the scientific CCD in either a full frame pixel binning mode, $1 \times 1$ binning, with all $1056 \times 1030$ pixels, or a $2 \times 2$ binning with $528 \times 515$ pixels. The $2 \times 2$ binning mode results in the four combined pixels summing their collective detected signals, increasing the imaging cadence at the cost of a lower detection threshold, as well as halving the accuracy of the observation [17].

NEOSSat carried out a longer commissioning phase than was planned due to imaging performance being degraded from elevated read-out noise on the CCD, a problem not detected while undergoing ground testing. This elevated noise was a result of current ripple induced by the satellite's switching power supply. A solution was eventually found, but unfortunately this solution came with the cost of greatly increasing the CCD readout time from 15 to 83 seconds for a $1 \times 1$ binned image, and up to 20 seconds for a $2 \times 2$ image. This increased time requirement resulted in a change for the operations tempo, however, the solution implemented in September 2015 allowed the satellite to exit the commissioning phase and begin to complete its HEOSS mission in earnest [17]. 
For the remainder of this topic, all NEOSSat tasking will refer to the procedures followed in the HEOSS mission and will exclude the protocol for NESS. NEOSSat is scheduled by creating a tasking file for a specified day of the year. This tasking file is created by the mission planner in considering the needs of the NEOSSat science team. For example, if specific targets are required, a certain portion of the target orbit, or a specific image binning mode is desired, the planner will accomodate as necessary. This tasking is completed by means of utilizing the latest TLEs of the target object and calculating the resulting pointing vectors for NEOSSat. Once the tasking file is complete, the file is transmitted to the CSA's Satellite Operations Team (SatOps) in St-Hubert, Quebec to perform a quality control with the NEOSSat Constraint Checker (NCC). This system checks that any tasking files that are uploaded to the satellite do not violate any of the system limits in order to ensure that the satellite will not trip a condition that will cause the satellite to enter a safe mode. Once the files are checked, they are uploaded to the satellite.

Due to the very small area of the sky being observed in an image, as seen in Fig. 2.7, there is an important requirement to precisely know the position of a target object. When imaging an object in GEO, the FOV of NEOSSat is $549 \times 536 \mathrm{~km}$, a size sufficiently large enough to accomodate for the uncertainty in the associated TLE and significantly smaller than the mean orbital speed of three $\mathrm{km} / \mathrm{s}$ in that regime. If NEOSSat were to look at an object in close proximity, assuming a distance of $150 \mathrm{~km}$, the FOV is shrunk to $1.5 \times 1.5 \mathrm{~km}$, a sharp contrast to the size at GEO. In this case, the uncertainty of TLEs are still present, but their impact is greatly amplified due to the substantially closer distance, as well as other errors becoming more pronounced such as the associated position errors for NEOSSat as the observer. This narrow FOV requires NEOSSat to be tasked on the high-precision SP ephemerides from the USAF in order to allow the satellite to be able to successfully track an object at such close proximity.

The metric accuracy of the sensor was determined by using collected data during a period from 15 September 2015 to 3 February 2016. An observation campaign focussing on GPS satellites assessed the metric accuracy of the sensor in $1 \times 1$ and $2 \times 2$ bin collection modes. A total of 414 observations in $1 \times 1$ binning and 183 observations 


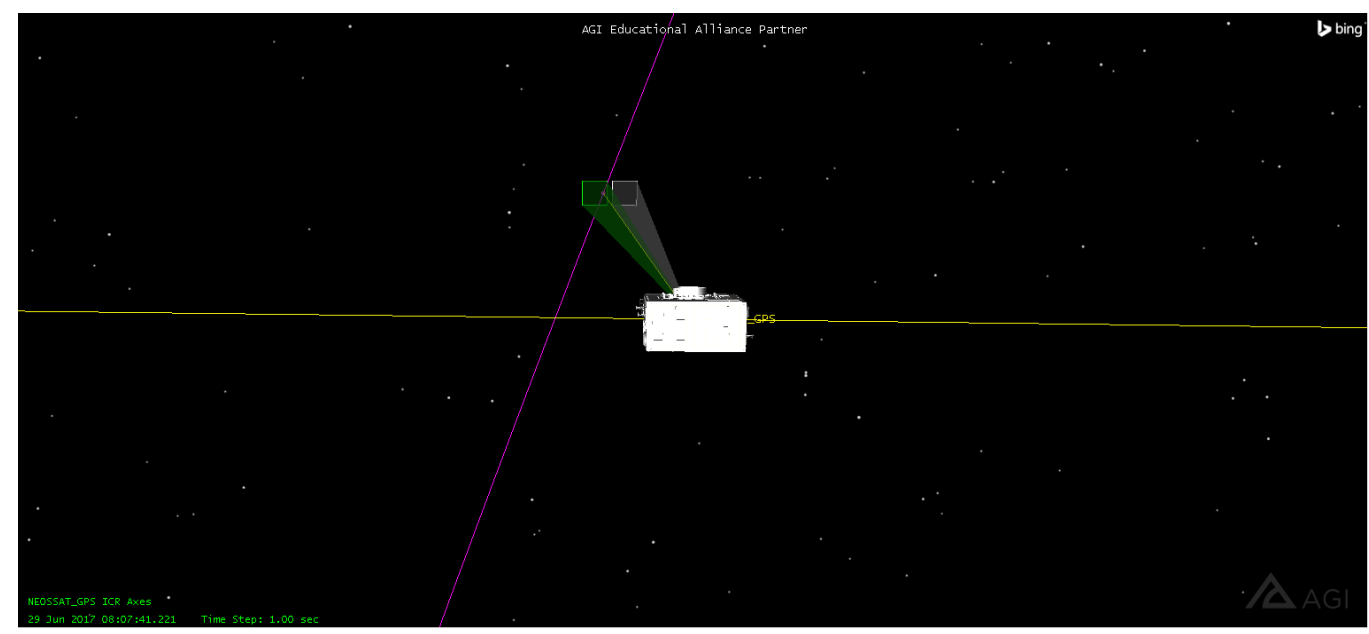

Figure 2.7: NEOSSat Science and Star Tracker CCD FOVs observing a GEO satellite

in $2 \times 2$ binning were collected over the four and a half month period. GPS satellites were selected as targets due to their designation as calibration satellites, or Calsats, where the daily 24-hour period high-precision ephemerides are available for download from the United States National Geodetic Service. These high-precision ephemerides are produced for numerous reasons, supporting studies in geodesy, surveying, and space surveillance [17].

The HEOSS scientific team developed an image processing software written in Mathwork's MATLAB. This system was named the Semi-QUIck Detection 3rd Iteration (SQUID3) and performed a wide variety of functions, including filtering out noise within raw images, identifying background star streaks, centroiding and correlating the star streaks to a star catalog to determine Right Ascension (RA) and Declination (DEC) angular information, as well as performing image stacking as required. Once processed by the SQUID3 program, the observations were ingested, alongside the high-precision GPS ephemerides, into AGI's Orbit Determination Tool Kit (ODTK). Within ODTK, the filterless mode was used to compute residuals between the published high-precision ephemerides and the NEOSSat observations [17]. The results from the GPS imaging campaign can be seen in Table 2.1, units in arcseconds.

Thorsteinson specifies the possible sources of error to include: timing, centroiding, observation corruption, observation format precision, as well as possible reasons for 
Table 2.1: GPS Campaign Metric Results (values in arcseconds) ${ }^{3}$

\begin{tabular}{ccccc}
\hline \hline Binning & $\begin{array}{c}\text { RA } \\
1 \times 1\end{array}$ & $\begin{array}{c}\text { DEC } \\
1 \times 1\end{array}$ & $\begin{array}{c}\text { RA } \\
2 \times 2\end{array}$ & $\begin{array}{c}\text { DEC } \\
2 \times 2\end{array}$ \\
\hline \hline$\sigma$ & 3.5 & 3.8 & 3.7 & 3.8 \\
Bias & 0.15 & -1.3 & 2.1 & 1.3 \\
\hline
\end{tabular}

the increased bias in the $2 \times 2$ imagery resulting from the scheduling of objects at similar times of the day [17].

\subsection{Relative Motion Dynamics}

Up to this point, discussion of orbits have all been with respect to either the ECEF or J2000 reference frames, which have a coordinate origin at the center of the Earth. However, for missions involving rendezvous and proximity operations, there is a need to relate the position of one object with respect to another while in orbit.

Much work describes the relative motion of objects, the most easily identifiable formulations are the Clohessy-Wiltshire, or Hill's equations [23]. These equations express a simplified, linearized description of one satellite's motion with respect to the other using two-body dynamics. Hill's equations assumes one object is in a circular orbit and the other is closely spaced. These equations are derived from the exact non-linear equations of motion of the relative dynamics between spacecraft $[6,24]$.

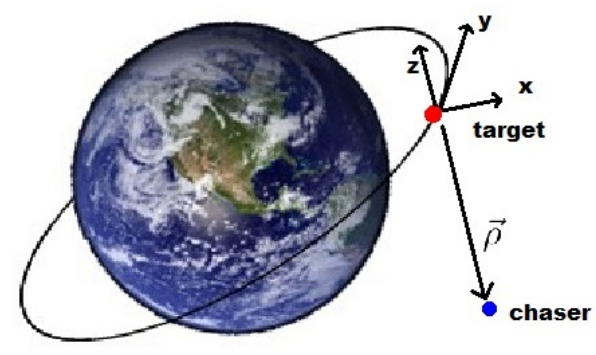

Figure 2.8: LVLH

The relative motion reference frame is called the Local-Vertical-Local-Horizontal $(L V L H)$, denoted as $\overrightarrow{\mathcal{F}}_{L}$, with an origin on the center of the target spacecraft with

\footnotetext{
${ }^{3} 1$ arcsecond is defined as $1 / 3600$ of a degree
} 
the chaser spacecraft orbiting about the center of the frame. This reference frame is shown in Fig. 2.8 and is described by it's vectrix $\overrightarrow{\mathcal{F}}_{L}$ :

$$
\overrightarrow{\mathcal{F}}_{L}=\left[\begin{array}{c}
\vec{L}_{x} \\
\vec{L}_{y} \\
\vec{L}_{z}
\end{array}\right]
$$

where $\overrightarrow{L_{x}}$ is in the direction of the target spacecraft's position vector $\vec{r}_{t}, \overrightarrow{L_{z}}$ being parallel to the orbit normal $\vec{h}$, and $\overrightarrow{L_{y}}$ completing the right-handed reference frame as follows [24]:

$$
\begin{gathered}
\vec{L}_{x}=\frac{\vec{r}_{t}}{r_{t}} \\
\vec{L}_{z}=\frac{\vec{h}}{h} \\
\vec{L}_{y}=\vec{L}_{z} \times \vec{L}_{x}
\end{gathered}
$$

The relative position vector between the two spacecraft in terms of the LVLH reference frame is given by:

$$
\vec{\rho}=\overrightarrow{r_{c}}-\overrightarrow{r_{t}}=\overrightarrow{\mathcal{F}}_{L}^{T}\left[\begin{array}{l}
x \\
y \\
z
\end{array}\right]
$$

where $\vec{\rho}$ is the relative position vector while $x, y$, and $z$ are the radial, along-track, and cross-track components respectively of this vector in LVLH.

Using simple vector addition, the position of the chaser, $\vec{r}_{c}$, can be defined as:

$$
\vec{r}_{c}=\vec{r}_{t}+\vec{\rho}
$$

The velocity and acceleration of the chaser can be found by applying the governing kinematic relations, while the velocity and acceleration of the chaser can be expressed in the LVLH reference frame as [24]:

$$
\dot{\vec{r}}_{c}=\stackrel{\circ}{\vec{r}}_{c}+\vec{\omega}_{L I} \times \vec{r}_{c}
$$




$$
\ddot{\vec{r}}_{c}=\stackrel{\circ}{\vec{r}}_{c}+\dot{\vec{\omega}}_{L I} \times \vec{r}_{c}+2 \vec{\omega}_{L I} \times \stackrel{\circ}{\vec{r}}_{c}+\vec{\omega}_{L I} \times\left(\vec{\omega}_{L I} \times \vec{r}_{c}\right)
$$

where:

$$
\begin{aligned}
& \vec{r}_{c}=\overrightarrow{\mathcal{F}}_{L}^{T}\left[\begin{array}{c}
r_{t}+x \\
y \\
z
\end{array}\right] \\
& \stackrel{\overrightarrow{\vec{r}}_{c}}{=} \overrightarrow{\mathcal{F}}_{L}^{T}\left[\begin{array}{c}
\dot{r}_{t}+\dot{x} \\
\dot{y} \\
\dot{z}
\end{array}\right] \\
& {\stackrel{\circ}{\vec{r}_{c}}}_{=}=\overrightarrow{\mathcal{F}}_{L}^{T}\left[\begin{array}{c}
\ddot{r}_{t}+\ddot{x} \\
\ddot{y} \\
\ddot{z}
\end{array}\right] \\
& \vec{\omega}_{L I}=\overrightarrow{\mathcal{F}}_{L}^{T}\left[\begin{array}{l}
0 \\
0 \\
\dot{\theta}
\end{array}\right] \\
& \dot{\vec{\omega}}_{L I}=\overrightarrow{\mathcal{F}}_{L}^{T}\left[\begin{array}{l}
0 \\
0 \\
\ddot{\theta}
\end{array}\right]
\end{aligned}
$$

where $\vec{\omega}_{L I}$ and $\dot{\vec{\omega}}_{L I}$ are the angular velocity and acceleration vectors of the LVLH reference frame with respect to the inertial reference frame, $\theta$ is the true anomaly of the target spacecraft, and $r_{t}$ is the magnitude of the target spacecraft's position vector, while $\stackrel{\dot{\vec{r}}_{c}}{\text { and }} \stackrel{\stackrel{\circ}{\vec{r}}}{c}$ are the first and second time derivatives of $\vec{r}_{c}$ in the LVLH reference frame [24]. When combined, the acceleration of the chaser is obtained by [24]:

$$
\ddot{\vec{r}}_{c}=\overrightarrow{\mathcal{F}}_{L}^{T}\left[\begin{array}{c}
\ddot{r}_{t}+\ddot{x}-\ddot{\theta} y-2 \dot{\theta} \dot{y}-\dot{\theta}^{2}\left(r_{t}+x\right) \\
\ddot{y}+\ddot{\theta}\left(r_{t}+x\right)+2 \dot{\theta}\left(\dot{r}_{t}+\dot{x}\right)-\dot{\theta}^{2} y \\
\ddot{z}
\end{array}\right]
$$


When assuming Keplerian motion, angular momentum is conserved and therefore this representation can be modified where $\ddot{r}_{t}$ and $\ddot{\theta}$ can be expressed as:

$$
\begin{gathered}
\ddot{r}_{t}=\dot{\theta}^{2} r_{t}-\frac{\mu}{r_{t}^{2}} \\
\ddot{\theta}=-2 \frac{\dot{r_{t}}}{r_{t}} \dot{\theta}
\end{gathered}
$$

Substituting the values for $\ddot{r}_{t}$ and $\ddot{\theta}$ into Eq. (2.15) gives:

$$
\begin{gathered}
\ddot{\vec{r}}_{c}=\overrightarrow{\mathcal{F}}_{L}^{T}\left[\begin{array}{c}
\ddot{x}-\dot{\theta}^{2} x-2 \dot{\theta}\left(\dot{y}-y \frac{\dot{r}_{t}}{r_{t}}\right)-\frac{\mu}{r_{t}^{2}} \\
\ddot{y}-\dot{\theta}^{2} y+2 \dot{\theta}\left(\dot{x}-x \frac{\dot{r}_{t}}{r_{t}}\right) \\
\ddot{z}
\end{array}\right] \\
\ddot{\vec{r}}_{c}=\overrightarrow{\mathcal{F}}_{L}^{T} \frac{\mu}{r_{c}^{3}}\left[\begin{array}{c}
r_{t}+x \\
y \\
z
\end{array}\right]
\end{gathered}
$$

Finally, when considering the two-body equations of motion given by Eq. (2.12) and modified into Eq. (2.19), the exact non-linear equations of motion of the chaser spacecraft with respect to the target can be described as:

$$
\begin{gathered}
\ddot{x}-\dot{\theta}^{2} x-\dot{\theta}^{2} \dot{y}-\ddot{\theta} y-\mu\left(\frac{r_{t}+x}{r_{c}^{3}}-\frac{1}{r_{t}^{2}}\right)=a_{x} \\
\ddot{y}-\dot{\theta}^{2} y+2 \dot{\theta} \dot{x}-\ddot{\theta} x+\frac{\mu}{r_{c}^{3}} y=a_{y} \\
\ddot{z}+\frac{\mu}{r_{c}^{3}} z=a_{z}
\end{gathered}
$$

where $\mu$ is Earth's constant gravitational parameter, and $a_{x}, a_{y}$, and $a_{z}$ are the accelerations in the radial, along-track, and cross-track components respectively of this vector in LVLH.

In order to simplify the equations, the system can be expressed in state space representation defined in Eqs. (2.23) - (2.24). Equation (2.23) represents the dynamics 
of the system over time, where $A$ is the state matrix which describes the dynamics of the system, $X$ is the state vector with the different system states, $B$ is the input matrix which describes the reaction of the system to the effect of an input, $u$. Equation (2.24) represents the outputs of a system, $Y$, from the state vector by means of the output matrix, $C$, which translates the states of the system to the ones that can be directly measured.

$$
\begin{gathered}
\dot{X}(t)=A X(t)+B u(t) \\
Y(t)=C X(t)
\end{gathered}
$$

Using the relative motion equations in state space representation, with the assumption that neither spacecraft can manoeuvre (therefore $u=0$ ), the state vector and the state transition matrix are given by:

$$
\begin{aligned}
& X=\left[\begin{array}{c}
x \\
y \\
z \\
\dot{x} \\
\dot{y} \\
\dot{z}
\end{array}\right] \\
& A=\left[\begin{array}{cccccc}
0 & 0 & 0 & 1 & 0 & 0 \\
0 & 0 & 0 & 0 & 1 & 0 \\
\dot{\theta}^{2}-\frac{\mu}{r_{c}^{3}} & \dot{\theta}^{2}-\frac{\mu}{r_{c}^{3}} & 0 & -2 \dot{\theta} & 0 & 0 \\
-\ddot{\theta} & 0 & -\frac{\mu}{r_{c}^{3}} & 0 & 0 & 0
\end{array}\right] \\
& 0 \\
& 0
\end{aligned}
$$

\subsection{Observability}

In state estimation theory, the concept of observability is if the measureable external outputs from a system allows for a unique estimation of the non-measureable internal 
states [25].

Observability is often easily explained and tested when the state of the system is described in state space representation. The unforced state space in Eqs. (2.27) - (2.28) where $\mathrm{X}$ is the $n \times 1$ state vector $(n, 1 \in \mathbb{R}), A$ is the $n \times n$ state matrix describing how the system evolves, $y$ is the $p \times 1$ system output $(p \in \mathbb{R})$, and $C$ is the $p \times n$ output matrix describing how the internal states are translated to the system output.

$$
\begin{aligned}
& \dot{X}(t)=A X(t) \\
& Y(t)=C X(t)
\end{aligned}
$$

In order to determine if all the internal states are observable, a new matrix called the observability matrix, $O$, is examined to determine its rank:

$$
O=\left[\begin{array}{c}
C \\
C A \\
C A^{2} \\
\vdots \\
C A^{n-1}
\end{array}\right]
$$

The rank of a matrix is the number of linearly independent rows residing within that matrix. By having each row of the matrix be linearly independent, there is only one system state that can be associated for each output, and therefore the state is able to be uniquely identified. In this case, for the $n^{2} \times n$ observability matrix $O$, the system is observable if, and only if, its rank is equal to $n$ as follows:

$$
\operatorname{rank}(O)=n
$$

For space surveillance applications, this concept can be applied by visualizing two co-orbital spacecraft who share an identical orbit normal, meaning both move in the exact same plane. If the chaser was to observe the target, since they are both in the same plane, the chaser would not be able to ascertain any information regarding the target's out-of plane motion. This problem holds true for any number of subsequent 
observations taken of the target. For space surveillance purposes, particularly spacebased surveillance, this is often not an issue as spacecraft rarely share identical orbit normals, and missions are designed to surveil particular orbit classes where this issue is minimized. Despite this advantage, this problem still exists as all orbits must cross the equator and orbit planes do by nature intersect.

The observability rank criteria can be applied to a simplified space surveillance example for demonstration. Assuming that the target spacecraft is in a circular orbit in the $x-y$ plane with an altitude of $800 \mathrm{~km}$, Eq. (2.26) can be simplified to Eq. (2.31) by substituting $\theta=0, \dot{\theta}=\frac{2 \pi}{T}$, and $\ddot{\theta}=0$, where $T$ is the orbital period of the target spacecraft.

$$
A_{\text {test }}=\left[\begin{array}{cccccc}
0 & 0 & 0 & 1 & 0 & 0 \\
0 & 0 & 0 & 0 & 1 & 0 \\
0 & 0 & 0 & 0 & 0 & 1 \\
0 & 0 & 0 & 0 & 2.076 E-3 & 0 \\
0 & 0 & 0 & -2.076 E-3 & 0 & 0 \\
0 & 0 & -1.077 E-6 & 0 & 0 & 0
\end{array}\right]
$$

Assuming that the chaser can uniquely determine range to the target, then the chaser will be measuring the slant range to the target, $\vec{\rho}$. The slant range, as defined in Eq. (2.7), can be broken down into the $x, y$, and $z$ components:

$$
\left[\begin{array}{c}
\rho_{x} \\
\rho_{y} \\
\rho_{z}
\end{array}\right]=\left[\begin{array}{l}
r_{c_{x}} \\
r_{c_{y}} \\
r_{c_{z}}
\end{array}\right]-\left[\begin{array}{l}
r_{t_{x}} \\
r_{t_{y}} \\
r_{t_{z}}
\end{array}\right]
$$

Knowing that the target's orbit is in the $x-y$ plane with a true anomaly of 45 degrees, the position vector in kilometers of the target spacecraft is:

$$
r_{t}=\left[\begin{array}{c}
5076.12 \\
5076.12 \\
0
\end{array}\right]
$$

Assuming the chaser spacecraft is in the $y$-z plane with an altitude of $786 \mathrm{~km}$, 
similar to NEOSSat, a nominal position vector in kilometers of the chaser spacecraft when it passes through the $x-y$ plane is:

$$
r_{c}=\left[\begin{array}{c}
0 \\
7163 \\
0
\end{array}\right]
$$

The resulting slant range vector is now:

$$
\rho=\left[\begin{array}{c}
-5076.12 \\
2086.88 \\
0
\end{array}\right]
$$

This finding for the slant range vector shows that only the $x$ and $y$-component position information can be detected by the chaser spacecraft in this position. As only these two components can be measured, the output matrix, $C$, can be written as:

$$
C_{\text {test }}=\left[\begin{array}{llllll}
1 & 0 & 0 & 0 & 0 & 0 \\
0 & 1 & 0 & 0 & 0 & 0 \\
0 & 0 & 0 & 0 & 0 & 0
\end{array}\right]
$$

When creating the observability matrix as per Eq. (2.29), and applying the rank criteria as per Eq. (2.30), the rank of $O$ is 4 . As the number of observable states of $O$ is less than $n$, it can be concluded that this orbital configuration is only partially observable, where the $z$-components in position and velocity are unobservable. This orbital configuration can be visualized in Fig. 2.9 where the red and blue planes represent the orbit planes for the target and chaser respectively with a representative green sphere depicting Earth.

\subsection{Relative Orbit Determination}

It is important to first highlight the difference between initial inertial orbit determination (IIOD) and initial relative orbit determination (IROD) [13]. IIOD is the classic and well-studied orbit determination problem which will be discussed in Section 3.2. 


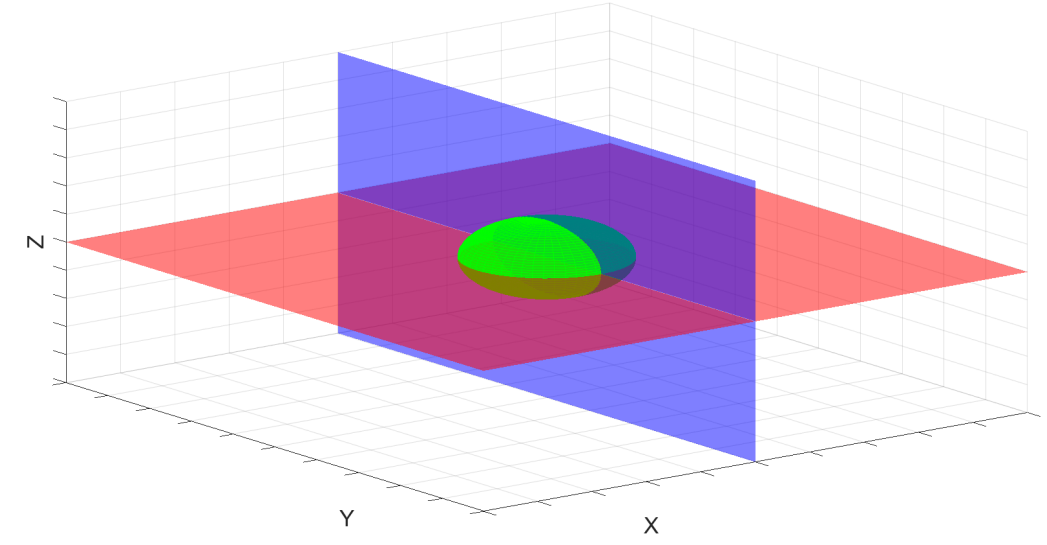

Figure 2.9: Target (red) and Chaser (blue) orbit planes intersecting

IROD is the orbit determination process of an object moving relative to the center of mass of another object while both are moving in orbit about a larger body as described in Section 2.4. Effectively, IROD can be performed using a closed form solution, while IIOD requires iterative methods. In the IROD case, the relative state vector of the object is generated as opposed to the inertial state vector. In both cases, for anglesonly measurements such as with optical systems, no range information is generated. This means that for IROD, the minimum amount of observation information required for IIOD techniques, three observations containing azimuth and elevation, is insufficient to uniquely describe the state. This fact is known as Woffinden's Dilemma [11]. In addition to this inability to provide sufficient data for a relative orbit with three angles-only measurements, this is true for any number of subsequent observations provided both objects are in unforced motion [1].

\subsubsection{Relative Orbit Observability}

In addition to the observability rank criteria outlined in Sec 2.5, the closed form solution of the linearized equations of motion can be used to convey the observability issue. In order to visualize this concept, the linearized equations of motion for a spacecraft about an arbitrary point as a system of linear equations is described in Eq. (2.37) where any future states of the spacecraft, $X\left(t_{1}\right)$ can be expressed as a function of the state transition matrix, $A$, and the initial state, $X\left(t_{0}\right)$. The assumptions under this linearized state solution require the spacecraft to be in relatively close proximity, 
but the closed form solution is able to be extended to handle nonlinear systems with nonlinear terms and disturbances as well [1].

$$
X\left(t_{1}\right)=A\left(t_{1}, t_{0}\right) X\left(t_{0}\right)
$$

Equation (2.37) can be broken down into:

$$
\left[\begin{array}{l}
r_{1} \\
v_{1}
\end{array}\right]=\left[\begin{array}{ll}
A_{r r} & A_{r v} \\
A_{v r} & A_{v v}
\end{array}\right]\left[\begin{array}{l}
r_{0} \\
v_{0}
\end{array}\right]
$$

Objects in relative orbits can be classified by their shape parameter, $P$, which is a ratio of their extreme maximum, $x_{b}$, and minimum, $x_{a}$, relative altitude values as per Eq. (2.39) where the absolute value of $x_{a}$ is smaller than $x_{b}$. Equation (2.39) results in a possible series of values for $P$ of $-1 \leq P \leq 1$. These shape parameters describe the relative motion in the LVLH reference frame as seen in Fig. 2.10.

$$
P=\frac{x_{a}}{x_{b}}
$$

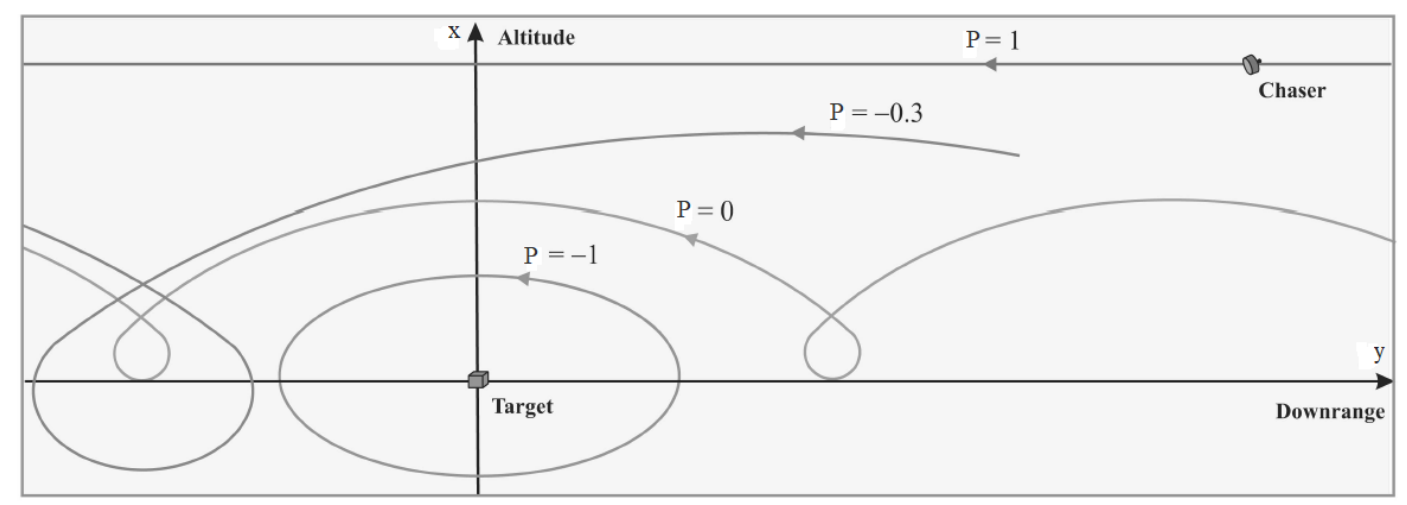

Figure 2.10: Shape Parameter [1] (modified)

If a LOS vector is defined between the two spacecraft as per Eq. (2.40), and is then combined with the general linearized state equation in Eq. (2.38), the LOS unit vector can be represented in Eq. (2.41).

$$
i_{r}(t)=\frac{r(t)}{|r(t)|}
$$




$$
i_{r}(t)=\frac{A_{r r}\left(r_{0}\right)+A_{r v}\left(v_{0}\right)}{\left|A_{r r}\left(r_{0}\right)+A_{r v}\left(v_{0}\right)\right|}
$$

The LOS vector can then be seen to have ambiguity due to scaling as a result of $P$ as seen in Eq. (2.42).

$$
i_{r}(t)=\frac{A_{r r}\left(P r_{0}\right)+A_{r v}\left(P v_{0}\right)}{\left|A_{r r}\left(P r_{0}\right)+A_{r v}\left(P v_{0}\right)\right|}=\frac{A_{r r}\left(r_{0}\right)+A_{r v}\left(v_{0}\right)}{\left|A_{r r}\left(r_{0}\right)+A_{r v}\left(v_{0}\right)\right|}
$$

The system is unobservable due to the impossibility to distinguish which scaled initial condition, $P X\left(t_{0}\right)$, produced the observed measurements, as seen in the identical LOS unit vector for any scaling value of $|P|>0$.

\subsubsection{Relative Orbit Observability Solutions}

As discussed previously, despite the issue of observability, Woffinden proposes practical solutions to overcome this dilemma [11] such as:

1. A properly calibrated set of manoeuvres to change the relative LOS vector,

2. Using a-priori knowledge of the shape and size of an object as well as the observed dimensions of the spacecraft to infer distance,

3. A camera offset from the center of mass of the spacecraft,

4. Utilizing a constrained reference frame, and

5. A second camera to employ stereo vision.

If properly calibrated manoeuvres can be performed, the range ambiguity, $P$, can be determined, and therefore, lead to a unique solution and an observable system [1]. The system becomes observable by performing manoeuvres that alter the LOS vector to enable estimating the scaling value, $P$. Visualized in Fig. 2.11, manoeuvres in (a) do not alter the LOS vector, while manoeuvres in (b) do. Depending upon the different shape parameters, there will be differing conditions for observability with the associated manoeuvres. There are even efforts currently underway to build relative navigation tools using angles-only measurements in conjunction with manoeuvre capabilites for LEO servicing spacecraft [26]. It is important to note that 
the observability issue relies on perfect measurements [1]. In the real world, perfect measurements are rare and as such it is possible that real world observations can potentially lead to the apparent observability of a non-observable nonlinear system and vice versa due to system noise.

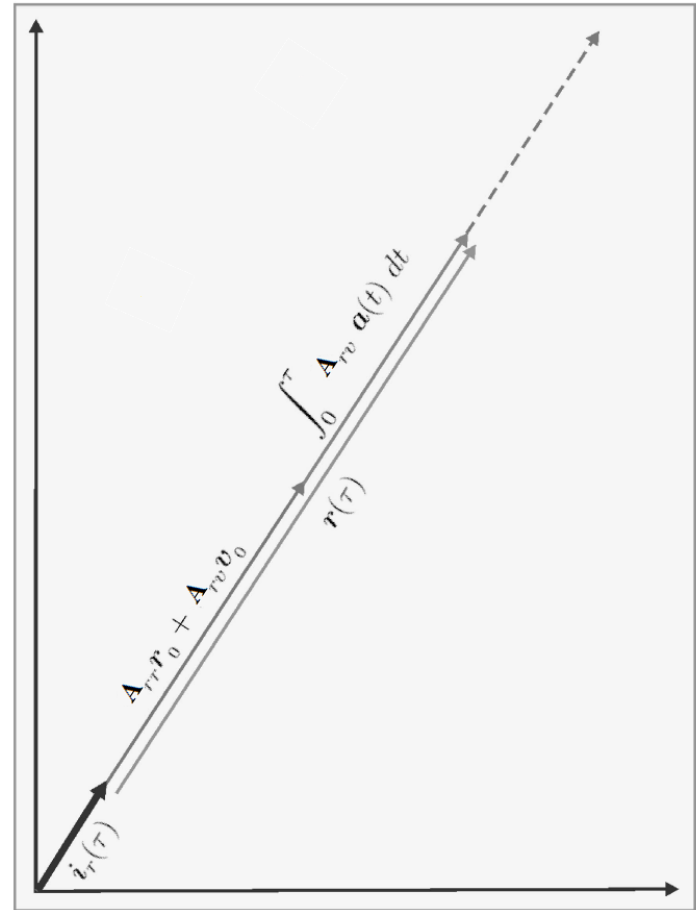

(a)

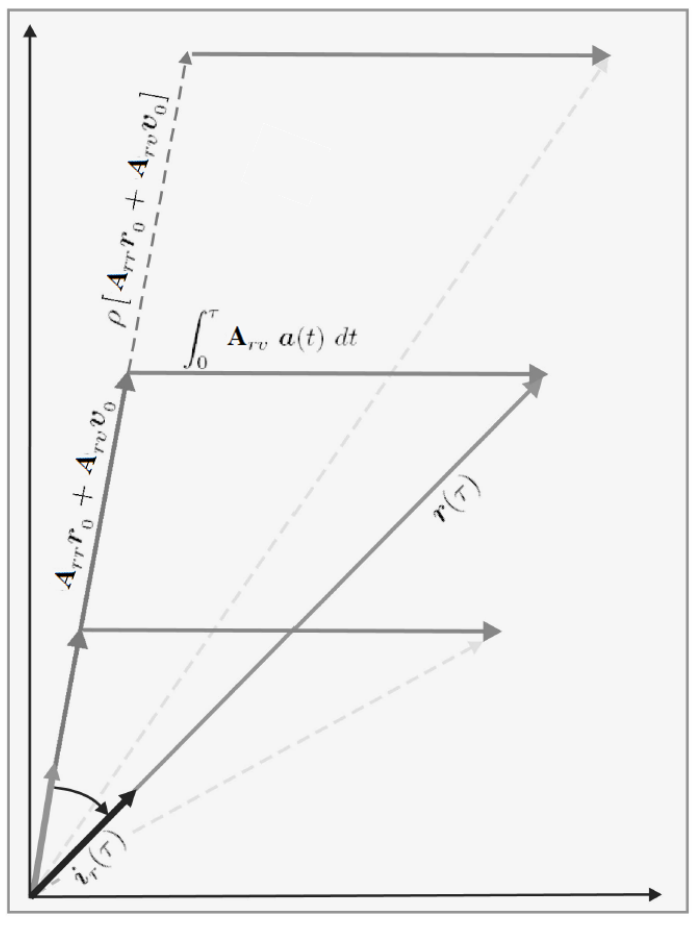

(b)

Figure 2.11: LOS altered by manoeuvres [1] (modified)

If the size and shape of an object is known in advance, it is possible to use this information to infer a coarse range measurement. By comparing the angular size of the observed object, simple trigonometry can be used to approximate distance to the objects $[12,13]$. Unfortunately, this is of limited practical value as it requires a cooperative entity to first share this data. This approach would best apply to active spacecraft, not debris, unless the shape and size of the debris is well known.

If the imaging system is offset from the primary satellite's center of mass, it is possible that sufficient parallax could be attained to achieve observability of the observed spacecraft's state $[12,13]$. This is advantageous as it negates the requirement to perform specially calibrated manoeuvres [12]. This parallax will effectively modify Eq. (2.41) by creating an offset, $d$, representing distance from the center of mass of 
the spacecraft seen in Eq. (2.43).

$$
i_{r}(t)=\frac{r(t)+d(t)}{|r(t)+d(t)|}
$$

In utilizing Eq. (2.43) as a basis for a modified Eq. (2.41), for the conditions when $d(t) \neq 0$, (ie, when there is an offset from the center of mass) then the problem can be expressed in the form of Eq. (2.44), demonstrating that observability is possible.

$$
i_{r}(t)=\frac{A_{r r}\left(r_{0}\right)+A_{r v}\left(v_{0}\right)+d(t)}{\left|A_{r r}\left(r_{0}\right)+A_{r v}\left(v_{0}\right)+d(t)\right|}
$$

Utilizing this camera offset, the resulting relative orbit quality is proportional to the measurement error of the sensor and inversely proportional to the ratio of the camera offset to the separation distance between the two spacecraft. For applications in LEO, camera offsets of $<1 \mathrm{~m}$ may be helpful for proximity operations with range separations of $<100 \mathrm{~m}$, but large offsets of approximately $50 \mathrm{~m}$ are required to extend that accuracy for several kilometers [13]. This result effectively precludes the use of offset cameras for proximity operations between small spacecraft. Also of importance is the conclusion from previous work that in order to accommodate for sensors with measurement error (ie. noise), observations must be separated by hundreds of seconds in LEO in order to achieve useful solutions [13].

It is theoretically possible to obtain observability in a constrained orbit system. Work has been done by acknowledging Woffinden's dilemma, but hypothesizing that the observability problem is solved by introducing the curvature of the orbit through the angular component of a specified cylindrical coordinate system. In order to facilitate understanding, the emphasis is on the two-dimensional problem where two spacecraft are in the same plane and therefore share an identical orbit normal. The shared orbit normal of the chaser and target spacecraft form the $z$ component of the coordinate system, while the $x$ and $y$ components are inertially fixed to the center of the primary gravitational body. The position vector of the chaser, $\rho_{c}$, the target, $\rho_{t}$, and the vector from the target to the chaser, $\rho_{\text {rel }}$, along with their respective angular positions, $\theta_{c}, \theta_{t}$, and $\theta_{\text {rel }}$ can be seen in Fig. 2.12.

In this system, there are only four elements needing to be solved for the initial 


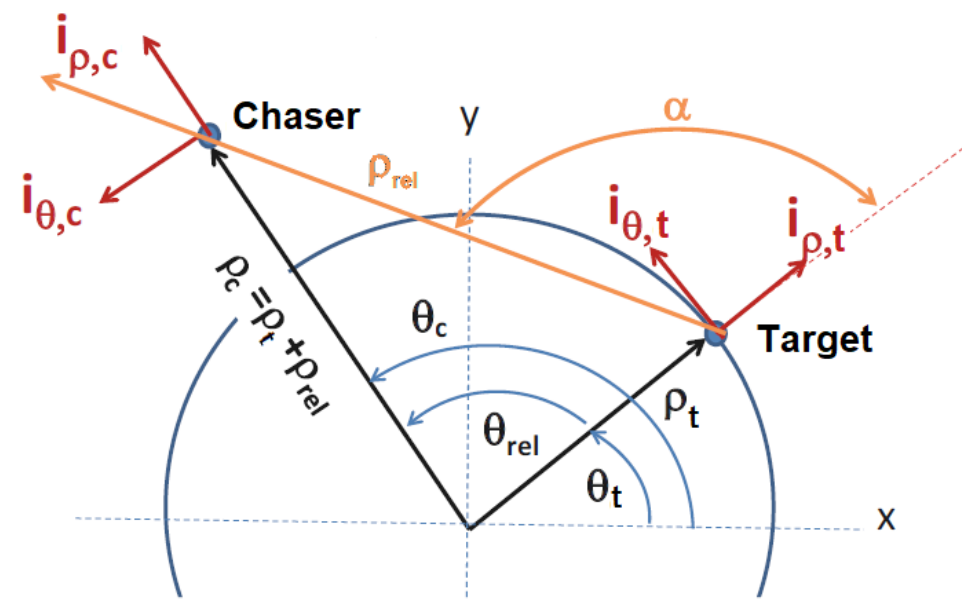

Figure 2.12: Constrained Cylindrical Coordinates [2] (modified)

relative state vector as there is no motion in the orbit normal component. The introduction of a measured observation angle, $\alpha$, becomes the single piece of information in each observation, which results in only four measurements needed to uniquely solve for the initial conditions of the relative state vector [2].

In the formulated cylindrical coordinate system, each LOS measurement from the chaser to target spacecraft is unique for each angular displacement of $\alpha$ for the pair. It is theorized that it may even be possible to uniquely determine range to the target spacecraft for the leader-follower pair in contrast to Woffinden's dilemma. It is also important to note that Geller and Lovell's work acknowledges that this coordinate system may be able to provide observability while using the nonlinearized equations of motion [2].

In the case of cylindrical coordinates, it was found that the optimal time interval between measurements to minimize the along-track position error and to optimize the relative state vector was slightly less or greater than one half orbit period. This was due to a significant decrease in the quality of the measurements at the onehalf orbit period. Although acknowledged in Geller and Lovell's work that a rigorous observability proof was not provided, evidence suggests that the problem is observable with the properly formulated cylindrical coordinate system [2].

Although there is a consistency amongst authors to utilize similar methods to 
solve the IROD problem, not many could be found which examined the possibility of using IIOD techniques in order to solve the IROD problem. This is likely due to the complexity involved with the IIOD process. Indeed, as stated recently:

"Theoretically, Gauss' method can be applied to this problem provided that the chaser's positions are known and substituted for the Earth-based observer position, however, Gauss' method is iterative in nature and has no known closed-form solution" [5].

This statement opens the possibility for space-based space surveillance platforms working in an inertial frame to detemine relative orbit information of another spacecraft in proximity. As spacecraft are uniquely designed for a specified mission, no space-based space surveillance platform is currently employed to perform this kind of task in an automated 'live' fashion. Therefore, any data processing and associated work on the subject must be conducted after the events and processed separately from the main mission objectives. 


\section{Chapter 3}

\section{Proposed Methodologies}

\subsection{Introduction}

This chapter outlines the inertial orbit determination process as well as the two major methodologies developed by the author when performing the inertial orbit determination process on NEOSSat observations. The first method used no a-priori orbit information to generate an orbit, and the second method used coarse orbit information of the target's position. In addition, an outline of the metric assessment of the satellite's observations will be included.

\subsection{Orbit Determination}

The process of orbit determination is to take the individual position measurements of an object in orbit at one time instance, and attempt to estimate the orbit that would have resulted in those observations. The process itself has been well studied over the years but is still an incredibly challenging process due to the iterative nature of current solutions. For the process of orbit determination, any representation of the state of an object, whether Keplerian elements or Cartesian coordinates, are both perfectly valid. For this work, Cartesian coordinates are used for ease of use with commercially available processing software. The estimated state of each object will take the form outlined in Eq. (3.1).

$$
X \equiv\left[\begin{array}{c}
x \\
y \\
z \\
\dot{x} \\
\dot{y} \\
\dot{z}
\end{array}\right]
$$


The estimated state of the object includes both the Cartesian position and velocity values. With angles-only measurements, such as space-based RA and DEC, as seen in Fig. 3.1, the measurements themselves contain no range information and therefore the actual position of the object even in one observation is unknown. This can be seen in Eq. (3.2) with the scalable range multiplier of $\rho$ providing ambiguity when converting the measured RA $(\alpha)$, and DEC $(\delta)$, values to Cartesian coordinates.

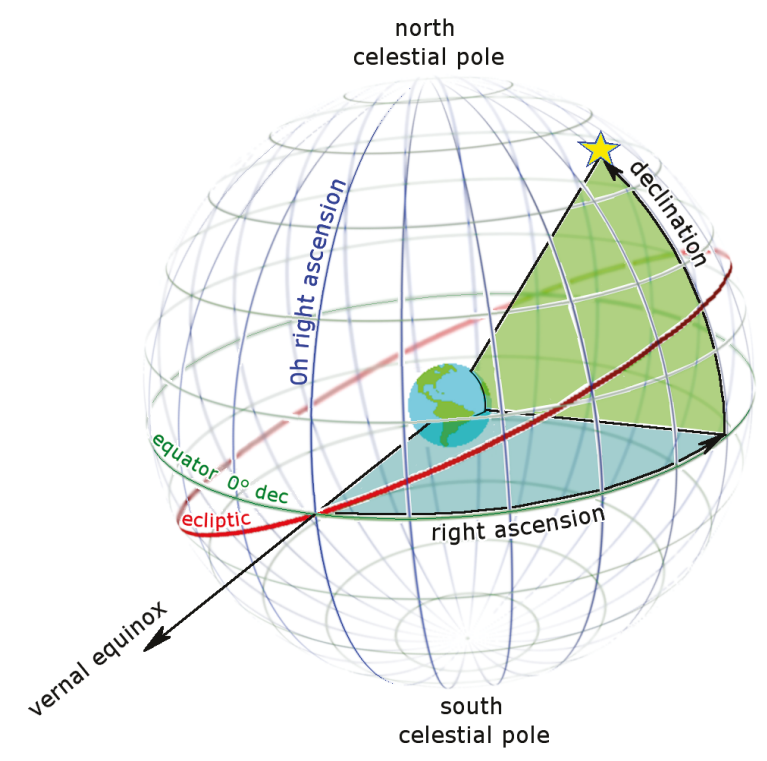

Figure 3.1: Right Ascension and Declination ${ }^{1}$

$$
\left[\begin{array}{l}
x \\
y \\
z
\end{array}\right]=\rho\left[\begin{array}{c}
\cos (\delta) \cos (\alpha) \\
\cos (\delta) \sin (\alpha) \\
\sin (\delta)
\end{array}\right]
$$

Functionally, orbit determination follows a hierarchy of Initial Orbit Determination (IOD), where a coarse state estimate is derived, followed by Differential Correction (DC) which allows for a more refined estimate to be produced. The goal of IOD is to simply provide a sufficiently close approximation in order to allow for DCs to converge to the correct state solution. The simplified description of the hierarchy of Orbit Determination is in reality, much more difficult. There are several complexities

\footnotetext{
${ }^{1}$ Right Ascension and Declination, 2012, (Accessed July 13, 2018), https://upload.wikimedia.org/wikipedia/commons/9/98/Ra_and_dec_on_celestia_sphere.png
} 
that must be understood in order to understand why this hierarchy exists, the first of which is the issue of the problem being non-linear in nature. This non-linearity requires linearization in order to employ linear estimation techniques such as Least Squares (LS) iteratively in the form of DCs. This use of LS first needs an initial orbit estimate to allow it to complete its task.

\subsubsection{Dynamics}

Orbit determination is a well-studied problem, many publications exist on the topic $[6,7]$. Orbit determination is a non-linear problem, making it inherently difficult to solve in its natural form. However, if the problem can be linearized about a point in time, such as at an observation, and if the observation can be related to the state vector, then linear state estimation techniques such as LS can be applied. The generic non-linear dynamics of the system can be expressed as a series of non-linear equations [7]:

$$
\begin{gathered}
\dot{X}=F(X, t) \\
Y_{i}=G\left(X_{i}, t_{i}\right)+e_{i}, i=1, \ldots, l
\end{gathered}
$$

where $X_{i}$ is the $n$-dimensional state vector such that $n \in \mathbb{R}$ at the time instant $t_{i}$, where $Y_{i}$ is the $p$-dimensional observations with $p \in \mathbb{R}$, where $e_{i}$ is noise at the time instant $t_{i}$, all while $F$ represents the dynamics of the systems and $G$ maps the system states to measureable outputs. It is important to note that it is very rare for observations to capture all the state information of an object in a single measurement. For example, optical systems will return angular information only, leaving a range ambiguity in measurements. Radars will generally return more position information than optical systems, such as angular and range measurements, but they can be unattractive due to the relative costs. In terms of the dynamics of the system, the above equations can be expressed in state space representation as follows:

$$
\begin{gathered}
\dot{X}(t)=A(t) X(t) \\
Y_{i}=H_{i}\left(X_{i}\right)+e_{i}, i=1, \ldots, l
\end{gathered}
$$


where $X$ is the $n$-dimensional state vector, $A$ is the $n \times n$-dimensional state transition matrix, $Y_{i}$ is the $p$-dimensional observed states in observation $i$, and $H_{i}$ is the $p \times n$ dimensional observation matrix which relates the state vector to the observed states.

\subsubsection{Initial Orbit Determination}

Initial Orbit Determination (IOD) is the first step in the Orbit Determination process. For the purposes of this work, only angles-only methods will be discussed. A modern method called Gooding Angles Only $[8,27]$ combines the early mathematical fundamentals with modern techniques and processes to provide robust IOD estimates.

Gooding's is a robust iterative method that can effectively solve Lambert's problem for the object provided correct measurements are selected. The method requires the observations to be sufficiently spaced apart in time to allow for different points in the orbit arc to be found and used. The method requires user-specified initial range estimates to be provided in conjunction with the angular information of the first and third observation. The result is two initial estimates of the line-of-sight (LOS) vectors from the observing spacecraft to the object, $l_{1}$ and $l_{3}$. These relative vectors from the observing spacecraft must be converted into the inertial object position vectors, by means of vector addition $\left(r_{1}=s_{1}+l_{1}\right)$. The LOS vectors are added to the position vectors of the observing spacecraft, $s_{1}$ and $s_{3}$, resulting in initial position estimates for the spacecraft of $r_{1}$ and $r_{3}[8]$.

Once the two inertial position vectors $r_{1}$ and $r_{3}$ are found, solving Lambert's problem allows for an initial estimate of the velocity for the object at the first observation $v_{1}$. The initial $r_{1}$ and $v_{1}$ estimate is propagated forwards to the time of the second observation to estimate $r_{2}$ and $v_{2}$. By completing similar vector addition, $\left(r_{2}=s_{2}+l_{2}\right)$, the estimated $l_{2}$ value is compared to the actual measured $l_{2}$ value to determine if the error is within tolerance, seen in Fig. 3.2. The user estimated range is then refined and the entire method is repeated iteratively in an attempt to minimize the error between the measured $l_{2}$ and estimated $l_{2}$. This process continues until the range estimate is sufficient to allow the error between the measured $l_{2}$ and estimated $l_{2}$ to be within tolerance as illustrated in Fig. 3.3 [8] .

In addition to the user-specified initial range estimates, it is also required that the 


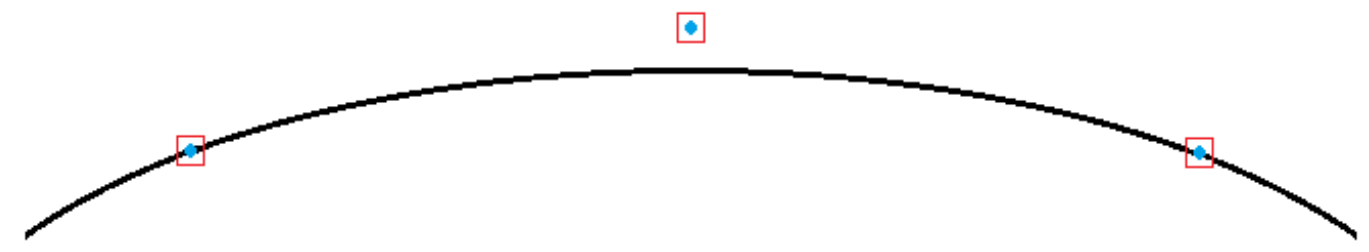

Figure 3.2: Initial Gooding Iteration

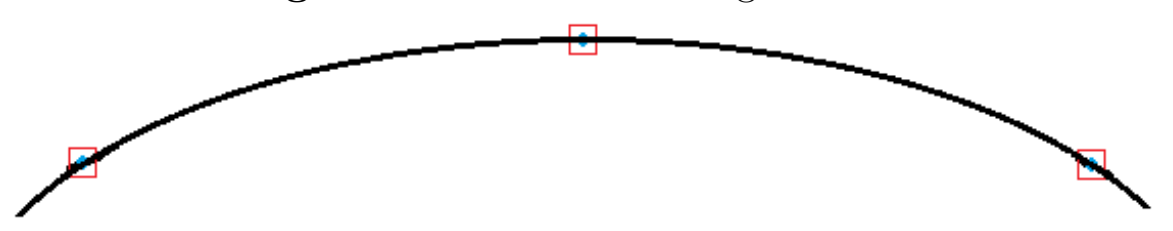

Figure 3.3: Final Gooding Iteration

user specify the number of half-orbit revolutions that the object completed during the time span from $l_{1}$ to $l_{3}$. The higher the number of half-revolutions, the more solutions will be found. However, only one solution will be the correct value and specifying the number of revolutions is important in order to decide on the correct solution.

\subsubsection{Least Squares}

Least squares is one of the simplest linear estimation techniques. The process consists of first identifying a model to describe a physical system, and then by using measurements, attempting to best fit the data to the model [6].

To begin, an appropriate force model must be selected. One issue is that although the relatively simple two-body and constrained three-body problem is completely determined, real world orbits are affected by other accelerations. There are currently many force models available to choose from, but none to the author's knowledge perfectly describe the real-world environment. A second issue is that for any known measurement system, there is an associated system noise that must be accommodated. A third issue, although less important with the advent of powerful computers, is the complexity of the model and associated memory which can be supported. This variety of issues has led to the standard procedures in space surveillance to approximate an object's orbit as close as possible and then update the orbits periodically in order to maintain orbit custody, preventing loss due to the mismatch between orbit 
propagation models and the physical reality.

A cost function to be optimized must be selected, this cost function will use errors between measurements and model predicted measurements, known as residuals, and denoted $e$. One of the most common cost functions, denoted $J$, allows for a minimum in the squares of the residuals to be found, that is:

$$
J(e)=\frac{1}{2} e^{T} e
$$

In expanding the equation with the substituted residuals, taking the derivative and setting to zero, so as to find the minimum, one can solve the equation in the form of Eq. (3.8) and then subsequently re-arrange to the form found in Eq. (3.9). This is the well-known least squares solution where $\hat{x}_{i}$ is the best estimate of the state, $y_{i}$ is the observed states at observation $i$, and $\tilde{H}$ is a mapping matrix relating $y_{i}$ to $\hat{x}_{i}$.

$$
\begin{gathered}
\left(\tilde{H}^{T} \tilde{H}\right) \hat{x}_{i}=\tilde{H}^{T} y_{i} \\
\hat{x}_{i}=\left(\tilde{H}^{T} \tilde{H}\right)^{-1} \tilde{H}^{T} y_{i}
\end{gathered}
$$

This method also allows for the estimation of the observation residuals, $\hat{e}_{i}$, defined as the estimate of the difference between observations and model predictions, alongside the best estimate of the state as follows:

$$
\hat{e_{i}}=y_{i}-\tilde{H}_{i} \hat{x}_{i}
$$

This is a useful qualitative and quantitative measure to examine the accuracy of the model fit to the observations. The application of LS to orbit determination is the fundamental basis of the optimal orbit determination process. The LS process outlined uses the simplest least squares cost function which allots equal weight to each observation. There are other cost functions which include weights to favour some measurements over others. Many least squares implementations for orbit determination use a weighted solution in order to reflect either a more accurate sensor in a network, or to prioritize newer observations over older ones. In addition, more than just the six state variables can be found, systems such as ODTK will solve for atmospheric drag values as well, provided proper drag models are included in the 
process.

\subsubsection{Linearization}

In order to apply LS to the process, the system dynamics must be linearized, requiring an initial state, $X_{0}$ to be known. If a reasonable trajectory is available, and if both the state vector, $X$, and the reference state vector, $X^{*}$, are relatively close throughout an observed time interval, then each of the terms in the non-linear system expressed in Eqs. (3.3) - (3.4) can be linearized by means of a Taylor series expansion [7] as follows:

$$
\begin{aligned}
& \dot{X}(t)=F(X, t) \\
&=F\left(X^{*}, t\right)+\left[\frac{\delta F(t)}{\delta X(t)}\right]^{*}\left[X(t)-X^{*}(t)\right]+\text { Higher Order Terms } \\
& Y_{i}=G\left(X_{i}, t_{i}\right)+e_{i} \\
&=G\left(X_{i}^{*}, t_{i}\right)+\left[\frac{\delta G(t)}{\delta X(t)}\right]_{i}^{*}\left[X\left(t_{i}\right)-X^{*}\left(t_{i}\right)\right]_{i}+\text { Higher Order Terms }
\end{aligned}
$$

This Taylor series expansion for each term of the state transition matrix, when ignoring the higher order terms, is equivalent to the Jacobian of the matrix. The Jacobian of the matrix $A$, denoted as $J_{A}$, can be calculated as follows:

$$
J_{A}=\left[\begin{array}{ccc}
\frac{\delta A_{1}}{\delta X_{1}} & \cdots & \frac{\delta A_{1}}{\delta X_{n}} \\
\vdots & \ddots & \vdots \\
\frac{\delta A_{n}}{\delta X_{1}} & \cdots & \frac{\delta A_{n}}{\delta X_{n}}
\end{array}\right]
$$

where $A$ is the $n \times n$-dimensional state transition matrix and $X_{i}$ is the $i$-th component of the state vector. This process can also be repeated to linearize the $p \times n$-dimensional observation matrix, $H$ : 


$$
J_{H}=\left[\begin{array}{ccc}
\frac{\delta H_{1}}{\delta X_{1}} & \cdots & \frac{\delta H_{1}}{\delta X_{n}} \\
\vdots & \ddots & \vdots \\
\frac{\delta H_{p}}{\delta X_{1}} & \cdots & \frac{\delta H_{p}}{\delta X_{n}}
\end{array}\right]
$$

where $J_{H}$ denotes the Jacobian of the matrix $H$. The resulting system is the linear approximation of the non-linear system and can also be expressed in state space representation as :

$$
\begin{gathered}
\dot{X}(t)=J_{A} X(t) \\
Y_{i}=J_{H}\left(X_{i}\right)+e_{i}, i=1, \ldots, l
\end{gathered}
$$

By examining Eq. (3.16), we can re-arrange the equation to identify errors in the observations, previously defined as the observation residuals, $\hat{e}_{i}$ :

$$
\hat{e}_{i}=y_{i}-\tilde{H}_{i}\left(x_{i}\right), i=1, \ldots, l
$$

In the particular case of RA and DEC measurements from Eq. (3.2) for a 6-state system $\left([x y z \dot{x} \dot{y} \dot{z}]^{\top}\right)$, the $p \times n$ dimensional $J_{H}$ can be expressed as a $2 \times 6$ matrix [19]. This expresses the sensitivity of the system states to changes in the measured states:

$$
J_{H}=\frac{1}{\rho}\left[\begin{array}{cccccc}
\frac{-\sin (\alpha)}{\cos (\delta)} & \frac{\cos (\alpha)}{\cos (\delta)} & 0 & 0 & 0 & 0 \\
-\cos (\alpha) \sin (\delta) & -\sin (\alpha) \sin (\delta) & \cos (\delta) & 0 & 0 & 0
\end{array}\right]
$$

\subsubsection{Differential Correction}

In the process of linearizing the non-linear system, we obtain an approximation of the system dynamics and the LS estimation will not obtain an exact solution as it would in a linear system. In order to find an exact solution, an iterative process called Differential Correction (DC) is used to attempt to improve the state estimate. The DC process uses iterative LS estimates to attempt to converge on the exact state solution. This process can be visualized as a $4^{\text {th }}$ order polynomial with two local minima in close proximity, one of which being the physically correct solution while the other 
being simply another mathematically correct one. The correct physical solution may be difficult to determine depending on the initial estimate of the state [6].

The radius of convergence is the distance from the correct physical solution which will allow the process to converge to the correct state estimate. If a user specifies an initial value outside that range, the solution will either diverge, or converge on an incorrect solution which is only mathematically correct. This is where the importance of getting a sufficiently close IOD estimate is crucial. If this initial state estimate is outside the radius of convergence, the correct physical solution will not be found.

The process of performing a differential correction involves selecting a slightly different state estimate from the initial or previous estimate, and then computing the associated residuals. The resulting residuals are used to determine how close the state estimate is to the actual solution by means of a root mean square (RMS) calculation defined by:

$$
R M S=\sqrt{\frac{1}{N} \sum_{i=1}^{N} \hat{e}_{i}^{2}}
$$

where $N$ is the number of observations used in the RMS process.

The nominal criteria for determining whether or not the solution has converged is to compare the difference between the current and previous RMS in order to confirm whether or not they are sufficiently close to consider the process to have converged. The acceptable tolerance to determine whether or not there is a converged solution, $\epsilon$, is a function of the non-linearity of the problem, as well as the measurement errors of the contributing instruments [6]. In practice, values for $\epsilon$ are very small, normally on the order of $1 \mathrm{E}-6$.

$$
\left|\frac{R M S_{i-1}-R M S_{i}}{R M S_{i-1}}\right| \leq \epsilon
$$

Once an orbit is determined from this process, the resulting state estimate is available alongside the uncertainy from the LS fit within the covariance matrix of the estimate. 


\subsubsection{Covariance Matrices}

From the LS process, the covariance matrix describes the variance in the state estimates as well as the correlation between the different state variables [28]. From Eq. (3.9), the covariance matrix, $P$, is defined as:

$$
P=\left(\tilde{H}^{T} \tilde{H}\right)^{-1}
$$

The covariance matrix is of $n \times n$ dimension where $n$ is the number of state variables estimated. The diagonal elements of $P$ are the variances of each state while the off-diagonal elements are the correlations between the different states along with a correlation coefficient, $\rho$. The correlation coefficient scales the correlation between changes in the state as being either positively or negatively related depending on the sign of the coefficient [28]. The covariance matrix will always be positive semidefinite symmetric, or positive definite symmetric.

$$
P=\left[\begin{array}{ccc}
\sigma_{x}^{2} & \rho_{x y} \sigma_{x}^{2} \sigma_{y}^{2} & \rho_{x z} \sigma_{x}^{2} \sigma_{z}^{2} \\
\rho_{x y} \sigma_{x}^{2} \sigma_{y}^{2} & \sigma_{y}^{2} & \rho_{y z} \sigma_{y}^{2} \sigma_{z}^{2} \\
\rho_{x z} \sigma_{x}^{2} \sigma_{z}^{2} & \rho_{y z} \sigma_{y}^{2} \sigma_{z}^{2} & \sigma_{z}^{2}
\end{array}\right]
$$

If observations used the in the LS process do not satisfy the observability criteria as specified in in Sec 2.5, the LS process will fail as the matrix $\tilde{H}^{T} \tilde{H}$ will be singular and as such, will be non-invertible.

\subsubsection{Sequential Processing}

Up to this point, the process has been focussed on taking observations and building the appropriate orbits. There is a useful technique, albeit difficult to implement, to simply update an already known orbit. This technique is called Sequential Processing.

In Sequential Processing, a-priori orbit information is available alongside a covariance matrix. This process involves utilizing an Extended Kalman Filter (EKF) which ingests observations and attempts to update the state estimate at the corresponding observation epoch. If an observation is within a certain error threshold, the filter will accept the observation to update the orbit estimate, otherwise if the measurement is 
too far from where the object is predicted to be, it is rejected and the filter simply propagates the state covariance forward [28].

The EKF is an extension of the linear Kalman Filter (KF) and used for non-linear problems. The KF is divided into two phases, predict and update. In the predict phase, the state estimate and covariance matrices are propagated to the observation epoch as follows:

$$
\begin{gathered}
\bar{X}_{i}=A \hat{X}_{i-1} \\
\bar{P}_{i}=A P_{i-1} A^{T}+Q
\end{gathered}
$$

where $\bar{X}_{i}$ is the predicted orbit state, $\hat{X}_{i-1}$ is the a-priori orbit state at the previous time step, $\bar{P}_{i}$ is the predicted covariance, $P_{i-1}$ is the a-priori covariance matrix, $A$ is the state transition matrix, and $Q$ is process noise.

A process noise signal must be inserted into the Kalman filter as per Eq. (3.24) due to the tendency for the associate covariance to shrink to zero. At first glance, one may like the idea of the matrix shrinking to zero as one would hope for perfect estimation. However, this poses a substantial problem called smugness where the filter itself will reject all subsequent observations as it incorrectly assumes a great deal of confidence in its prediction. The insertion of process noise forces the covariance to stay open and allow the filter to accept more observations.

In the update phase, the new observation at the next time step is ingested and both the state estimate and covariance matrix are updated as follows:

$$
\begin{gathered}
K_{i}=\bar{P}_{i} \tilde{H}_{i}^{T}\left(\tilde{H}_{i} \bar{P}_{i} \tilde{H}_{i}^{T}+R_{i}\right)^{-1} \\
\hat{X}_{i}=\bar{X}_{i}+K_{i}\left(y_{i}-\tilde{H}_{i} \bar{X}_{i}\right) \\
P_{i}=\left(I-K_{i} \tilde{H}_{i}\right) \bar{P}_{i}
\end{gathered}
$$

where $K_{i}$ is the gain on the Kalman filter, $y_{i}$ is the observed states at the next time step, $\tilde{H}_{i}$ is the measurement sensitivity matrix, and $R_{i}$ is the measurement noise 
matrix.

The EKF is based upon the same logic of the KF [28] and uses the linearized system dynamics outlined in Section 3.2.4.

\subsection{Method 1}

The first method assumes that no a-priori orbit information is available on the observed object. This technique can be used on objects which are found in the field of view of an observation, but perhaps are not tracked very well and no up-to-date TLEs are available. This methodology leverages the IOD techniques previously mentioned in Section 3.2.2 in order to gain an intial orbit estimate seen as $X_{\text {det }}$ in Fig. 3.4 to seed the DC process. The DC process as outlined in Section 3.2.5 can, if the IOD was within the radius of convergence, use a series of initial observations to produce a non-linear orbit estimate with a reasonable covariance seen as the outputs $\tilde{X}$ and $P$ respectively in Fig. 3.4. The orbit estimate from the non-linear dynamics that is converged upon through the DC process is more precise than the approximate solution for the linearized dynamics from the IOD. This orbit estimate and accompanying covariance can then be used to seed an EKF, as outlined in Section 3.2.7 which then allows for the processing of follow-on observations, with an output of a refined orbit, $\hat{X}$, and covariance $P$. The data flow for Method 1 can be seen in Fig. 3.4

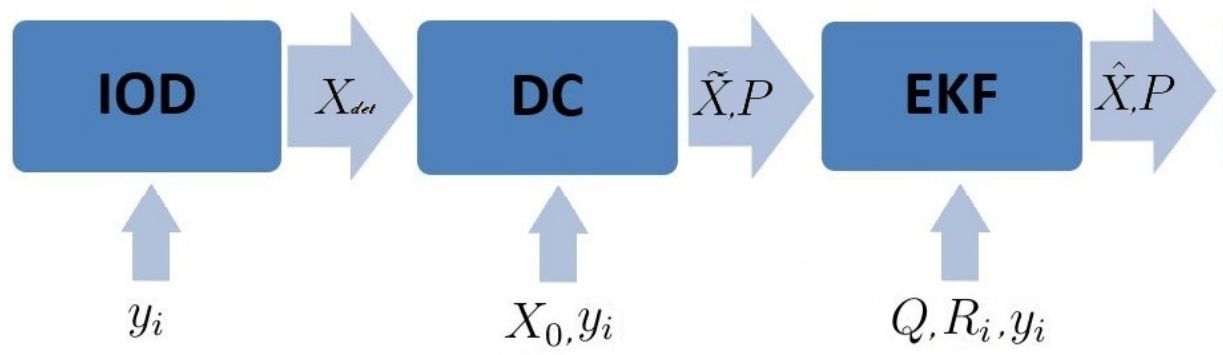

Figure 3.4: Method 1 Data flow 


\subsection{Method 2}

The second method uses available a-priori orbit information on the object such as special perturbation (SP) ephemerides or the most up-to-date TLEs. By initializing the estimate with complete orbit information, the IOD and DC steps can be avoided, leading to the direct seeding of the EKF with a non-linear orbit estimate, $\tilde{X}$ and a covariance $P$ as outlined in Section 3.2.7. Again, similar to Method 1, the output from the EKF is a refined orbit, $\hat{X}$, and covariance $P$. As SP ephemerides and TLEs generally do not have associated covariances, default covariances characterized by previous study in the field will be used to start the process [16]. This method is perhaps more practical to be implemented in a real-world application as the spacecraft would first need to be tasked to point towards the object to collect observations, a process requiring initial orbit information on the target. This second method is arguably more promising from an operational standpoint. Indeed, the chaser spacecraft would be able to immediately utilize the observations to update the orbit estimate, rather than wait for a series of observations that are seperated by a sufficient true anomaly to allow for IOD methods to start the OD process. The data flow for Method 2 can be seen in Fig. 3.5

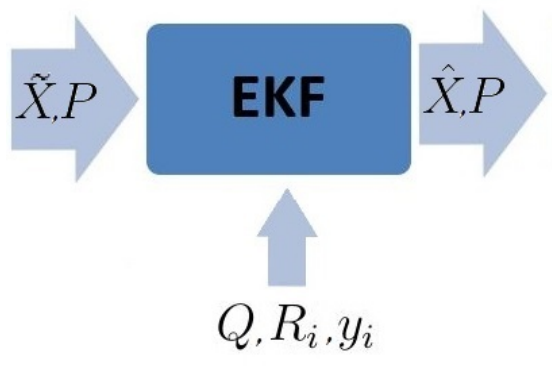

Figure 3.5: Method 2 Data flow

\subsection{Metric Assessment}

A metric accuracy assessment is the process of comparing a sensor's measurements to reference data. In the case of a space-based sensor such as NEOSSat, the collected RA and DEC measurements can be compared to the actual values at that time from 
the reference ephemerides of an observed RSO.

As an observation campaign targeting spacecraft in close proximity to NEOSSat rather than in the normal Deep Space regime had not been previously carried out by the sensor, there was no existing literature on the associated metric accuracy of the system on this class of object. Although a metric accuracy assessment was carried out on GPS satellites, it was unknown if the characteristic system noise would be the same, and if that value could be used as a valid starting point in order to ensure high-quality orbit fits. This consideration has immense impact on the overall process, particularly for Method 2, as the EKF requires knowledge of the sensor accuracy in the process to determine whether or not to accept observations to perform orbit updates.

In order to ensure the accuracy of this process, the assessment of metric accuracy of NEOSSat on objects in close proximity had to be carried out with accurate orbit reference ephemerides. Fortunately, one object in the observation campaign has publicly available high-precision ephemerides. The Satellite with ARgos and ALtika, or SARAL, has ephemerides produced by the International Laser Ranging Service (ILRS). The ILRS produces satellite and lunar ranging data in support of geophysical research. The service has a variety of ground stations worldwide which collect observations in an attempt to determine a high precision orbit estimate of the associated spacecraft. Daily ephemerides are available with $100 \mathrm{~m}$ 3D position accuracy [29], while post-fit ephemerides with one cm radial, cross-track, and five cm in-track accuracy are available with a 30-day delay [30]. In addition to NEOSSat's GPS ephemerides, which have a 3D 3-sigma threshold of sub one meter accuracy [31], this was the most accurate position information which could be identified to perform a metric assessment on the system noise. The metric accuracy assessment for NEOSSat would be performed using OD processing software by comparing the collected measurements to the truth ephemerides.

Unfortunately, the ILRS does not produce ephemerides for any of the other spacecraft which were observed by NEOSSat in this campaign. As a result, the metric assessment would need to be carried out solely on SARAL. In this case, the limited sample was used to establish the measurement noise properties of an EKF used to 
assess the system's accuracy. 


\section{Chapter 4}

\section{Experiment Setup}

\subsection{Introduction}

This chapter covers the overall experiment setup by outlining the close approach intervals for observations, the characteristics of the observed spacecraft, how NEOSSat was scheduled, the data processing for each observation interval, and an identification of the associated error sources.

\subsection{Close Approaches}

Each spacecraft deployed from a launch vehicle's upper stage is inserted into approximately the same orbit. Depending upon the orientation and the ejection mechanism aboard the launch vehicle, each satellite is imparted with a slightly different velocity, the difference in velocities between two objects being defined as $\Delta v$. The orientation of different spacecraft on a launch vehicle is determined by a variety of parameters, a few of the most important being shape, size, and mass. The spacecraft onboard the launch vehicle are ejected in a specific order depending upon how each spacecraft is attached to the launch vehicle. By ejecting the spacecraft sequentially, this allows for a physical separation and a corresponding level of safety of flight to be observed between each.

The variations in the imparted $\Delta v$ and the respective spacecraft masses result in slightly different orbital parameters. Although beginning with similar true anomaly values early in their orbit, these spacecraft begin to drift apart. In addition to these small orbit mis-matches, perturbing effects such as atmospheric drag, solar radiation pressure, and the J2 perturbation affect each spacecraft slightly differently based upon shape, size, and mass. This causes these spacecraft to occasionally come into proximity with each other as one drifts past the other. These periods of proximity are 


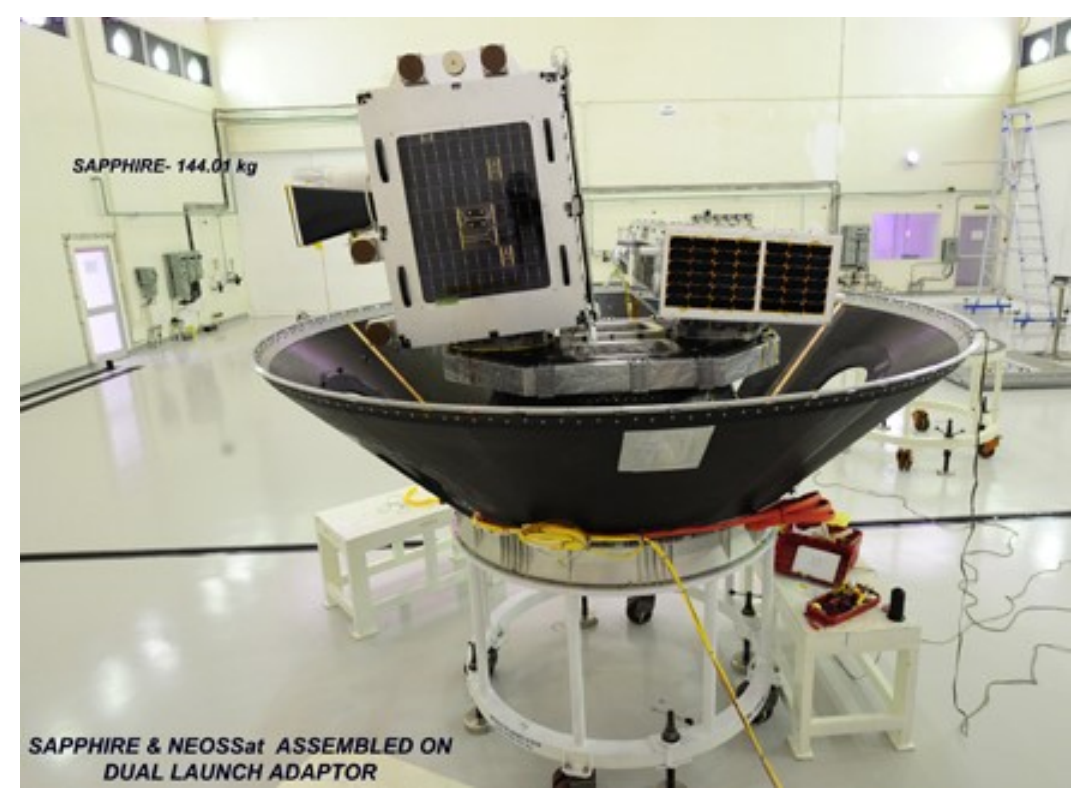

Figure 4.1: Sapphire and NEOSSat aboard the PSLV dual launch adapter ${ }^{1}$

herein defined as events, and are regular and easy to predict provided the spacecraft do not perform significant manoeuvres affecting it's semimajor axis $(a)$. The orbit parameters of the spacecraft which were launched onboard the Polar Satellite Launch Vehicle (PSLV) C20, as well as the difference in their semimajor axes compared to NEOSSat, are shown in Table 4.1, with an epoch of 10 Jun 2018 22:56:16.991 UTC:

Table 4.1: PSLV C20 Spacecraft Orbit Parameters

\begin{tabular}{ccccccc}
\hline \hline Object & $a(\mathrm{~km})$ & $\Delta a(\mathrm{~km})$ & $e$ & $i\left(^{\circ}\right)$ & $\Omega\left(^{\circ}\right)$ & $\omega\left(^{\circ}\right)$ \\
\hline \hline SARAL & 7160.132 & -1.093 & 0.00139 & 98.523 & 348.695 & 121.101 \\
AAUSAT3 & 7140.717 & -20.508 & 0.00167 & 98.543 & 8.263 & 80.968 \\
Sapphire & 7162.097 & 0.872 & 0.00051 & 98.536 & 5.543 & 45.576 \\
NEOSSat & 7161.225 & 0 & 0.00058 & 98.533 & 5.928 & 49.702 \\
STRaND-1 & 7141.344 & -19.881 & 0.00232 & 98.544 & 8.170 & 67.724 \\
BriteAustria & 7141.618 & -19.607 & 0.00244 & 98.540 & 7.449 & 58.362 \\
UniBRITE-1 & 7142.185 & -19.040 & 0.00154 & 98.540 & 7.116 & 319.547 \\
\hline \hline
\end{tabular}

${ }^{1}$ ISRO, PSLV-C20/SARAL Gallery, 2013, (Accessed Aug 23, 2018), https://www.isro.gov.in/pslv-c20-saral/pslv-c20-saral-gallery 


\subsection{Observed Spacecraft}

Six other satellites were launched with NEOSSat (SSN identification number: 39089) on its PSLV launch in 2013. In descending order by size, they were SARAL (39086), Sapphire (39088), BriteAustria (39091), UniBRITE-1 (39092), STRaND-1 (39090), and AAUSAT3 (39087). Each of the satellites from this launch undergo periodic proximity intervals with each other; five satellites from this launch made seven close approaches with NEOSSat and were observed over a time span from 29 June 2017 until 12 June 2018.

\subsubsection{SARAL}

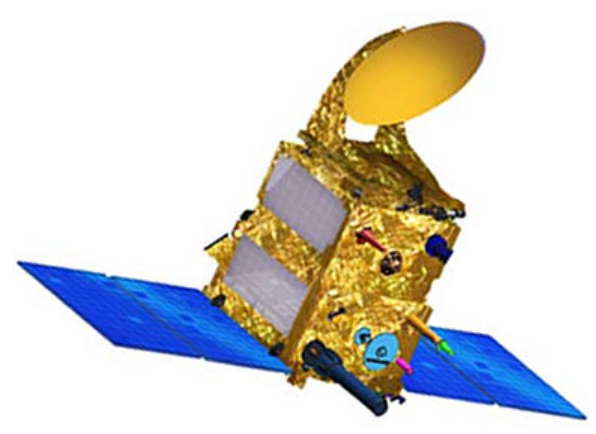

Figure 4.2: SARAL $^{2}$

The Satellite with ARgos and ALtika, or SARAL, is a joint Indian-French oceanographic survey mission which uses a Ka band altimeter to measure ocean surface elevation $^{3}$. With a beginning of life mass of $407 \mathrm{~kg}$ and an approximate size of $1 \times 1 \times 0.6 \mathrm{~m}^{4}$, SARAL is the only satellite which has an onboard propulsion system and periodically performs altitude station keeping manoeuvres. SARAL maintains its RAAN for a regular equator crossing time of 18:00 local time in support of its mission. This propulsion capability is in contrast to the other satellites from the C20 launch, which were all smaller and constructed without propulsion systems. By maintaining

\footnotetext{
${ }^{2}$ SARAL, 2013, (Accessed Aug 23, 2018), https://space.skyrocket.de/img_sat/saral__2.jpg

${ }^{3}$ ISRO, SARAL, 2013, (Accessed Jun 15, 2018), https://www.isro.gov.in/Spacecraft/saral

${ }^{4}$ SEOR, SARAL, 2018, (Accessed Jun 15, 2018), https://earth.esa.int/web/eoportal/satellite-missions/s/saral
} 
SARAL's RAAN, its orbit drifts relative to the others, where the RAAN of other satellites have all slowly changed over time. The difference between SARAL's orbit and that of NEOSSat, has resulted in the satellite partaking in more frequent revisit events with NEOSSat.

\subsubsection{Sapphire}

Sapphire is the first Canadian Space-Based Space Surveillance mission. Designed as a contributor to the US SSN, Sapphire houses a body-fixed optical imager with a similar operation mode to NEOSSat where it must change it's attitude to point and image objects. With a start of life mass of $148 \mathrm{~kg}$ and a size of $1 \times 1 \times 1 \mathrm{~m}$, the microsatellite is operated by MacDonald Dettweiler and Associates (MDA) under contract to DND. Of all the other satellites launched on PSLV C20, Sapphire is the most similar in size, mass, and purpose to NEOSSat.

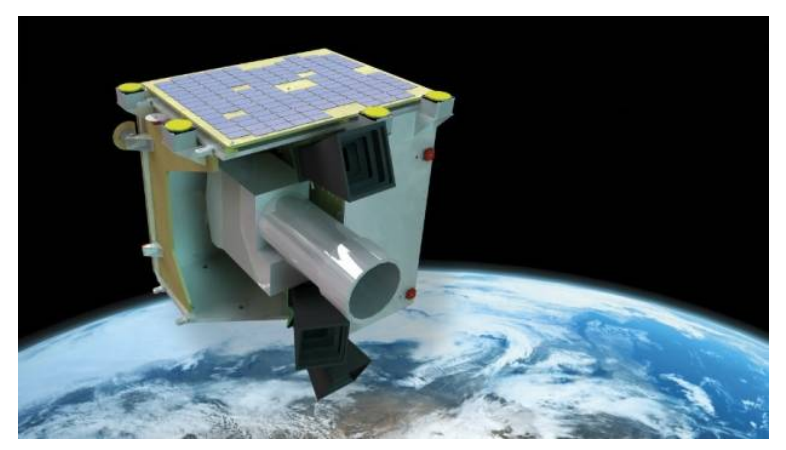

Figure 4.3: Artist's Rendition of Sapphire ${ }^{5}$

\subsubsection{BriteAustria}

BriteAustria, also known as CanX-3b, is part of the BRIght Target Explorer (BRITE) constellation. BriteAustria was the first Austrian satellite launched and was designed for an optical astronomy mission and was built by the University of Toronto's Space Flight Laboratoy (SFL). With a beginning of life mass of $<8 \mathrm{~kg}$ and a size of $20 \times 20 \times 20 \mathrm{~cm}$, the nanosatellite performs it's space-based astronomy mission of

\footnotetext{
${ }^{5}$ MDA, Sapphire, 2013, (Accessed Aug 23, 2018), http://www.asc-csa.gc.ca/eng/publications/space-policy/05-space-and-national-interest-2.asp
} 
imaging distant stars with a CCD. Alongside other satellites in the BRITE constellation, it examines the distant stars in an attempt to learn how stars interact with their local environment with the hopes to further our understanding of our own $\mathrm{Sun}^{6}$.

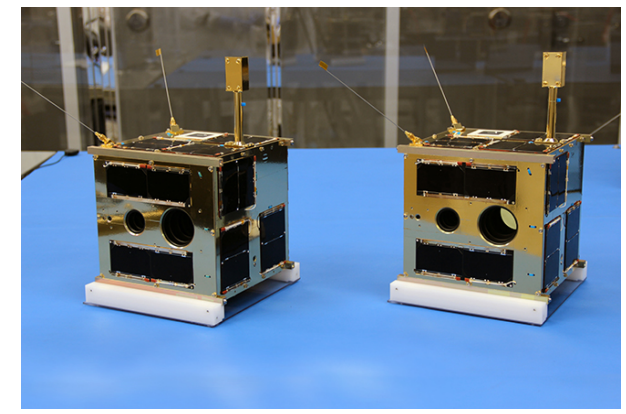

Figure 4.4: BriteAustria and UniBRITE ${ }^{7}$

\subsubsection{UniBRITE-1}

UniBRITE-1, also known as CanX-3a, is also part of the BRITE constellation and was also built by SFL. Constructed using an identical nanosatellite bus as BriteAustria with the same corresponding mass, the satellite is nearly identical to its sistersatellite. The difference between the satellites lies in the scientific payload where the two satellites image different portions of the visible spectrum, one in blue and one in the $\operatorname{red}^{8}$.

\subsubsection{AAUSAT3}

AAUSAT3 is a $10 \times 10 \times 10 \mathrm{~cm}$ cubesat designed by students from Aalborg University in Denmark. As the third cubesat from the university, it was designed to determine if it was possible for a $1 \mathrm{U}$ cubesat to detect ship-based Automated Identification System (AIS) signals in LEO. Of the satellites launched on PSLV C20, this satellite was the tiniest, and although lasting much longer than the design life, the satellite lost the ability to sufficiently re-charge its battery after nearly 19 months of operation,

\footnotetext{
${ }^{6}$ UTIAS, CanX-3 / BRITE-Constellation: Mission, 2014, (Accessed Jun 18, 2018), https://www.utias-sfl.net/?page_id $=407$

${ }^{7}$ Ibid.

${ }^{8}$ Ibid.
} 
subsequently ending its operational life ${ }^{9}$.

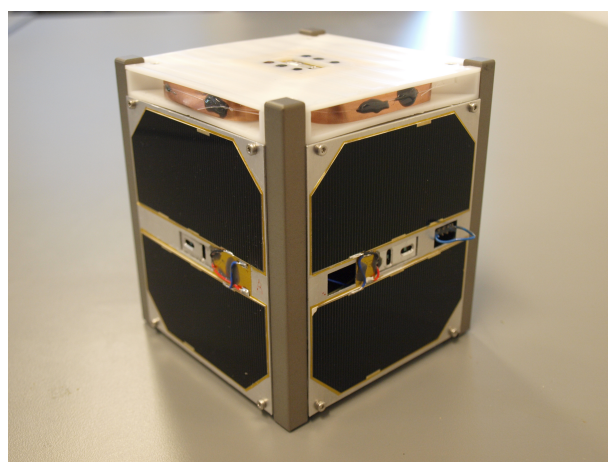

Figure 4.5: AAUSAT3 ${ }^{10}$

\subsubsection{QuikScat}

Not part of the PSLV C20 launch, QuikScat was a NASA Earth observation satellite designed to measure ocean surface wind conditions. Launched in 1999, the satellite utilized a microwave radar with a mechanically rotating dish antenna to sweep over the Earth's surface in regular intervals.

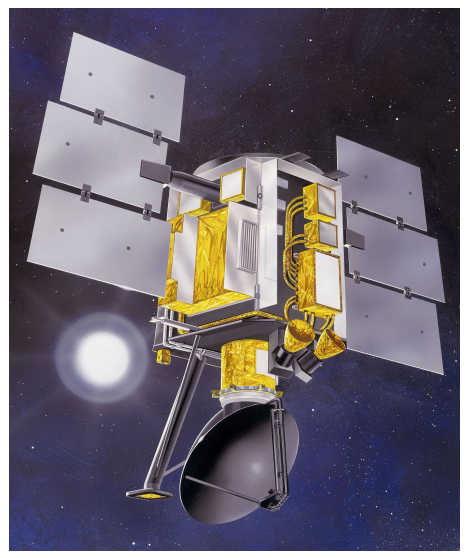

Figure 4.6: Artist's rendition of QuikScat. ${ }^{11}$

\footnotetext{
${ }^{9}$ Aalborg University, AAUSAT3, 2014, (Accessed Jul 23, 2018), http://www.space.aau.dk/aausat3/index.php?n=Tech.AAUSAT3InDetails ${ }^{10} \mathrm{Ibid}$.

${ }^{11}$ NASA JPL, QuikSCAT, 2009, (Accessed Jun 10, 2018), https://winds.jpl.nasa.gov/missions/quikscat/
} 
Designed for a 2 year mission, the satellite performed for 10.5 years prior to suffering a wear-induced mechanical failure tied to the antenna mechanism ${ }^{12}$. Coincidentally, QuikScat's orbit is very similar to that of SARAL, the primary difference is the true anomaly. Once this similarity was discovered by DRDC scientists, it became possible to task NEOSSat on the satellite in a similar manner to other satellites launched on the PSLV C20 mission. QuikScat's orbital parameters can be seen in comparison to those of SARAL, as well as the difference in their semimajor axes compared to NEOSSat, in Table 4.2, with an epoch of 10 Jun 2018 22:56:16.991 UTC:

Table 4.2: QuikScat Orbit Parameters

\begin{tabular}{cccccccc}
\hline \hline Object & $a(\mathrm{~km})$ & $\Delta a(\mathrm{~km})$ & $e$ & $i\left(^{\circ}\right)$ & $\Omega\left(^{\circ}\right)$ & $\omega\left(^{\circ}\right)$ & $\nu\left(^{\circ}\right)$ \\
\hline \hline NEOSSat & 7161.225 & 0 & 0.00058 & 98.533 & 5.928 & 49.702 & 132.295 \\
SARAL & 7160.132 & -1.093 & 0.00139 & 98.523 & 348.695 & 121.101 & 193.148 \\
QuikScat & 7170.915 & 9.690 & 0.00225 & 98.592 & 349.656 & 111.001 & 186.771 \\
\hline \hline
\end{tabular}

\subsection{Observation Events}

As previously discussed in Section 4.2, each of these satellites are aligned in such a way that they periodically come into close proximity with NEOSSat. This frequency is a result of the synodic period of the objects, and the repeating pattern is regular and easy to predict. The repeat pattern of the six observed satellites can be seen in Fig. 4.7 .

\footnotetext{
${ }^{12}$ NASA JPL, QuikSCAT, 2009, (Accessed Jun 10, 2018), https://winds.jpl.nasa.gov/missions/quikscat/
} 


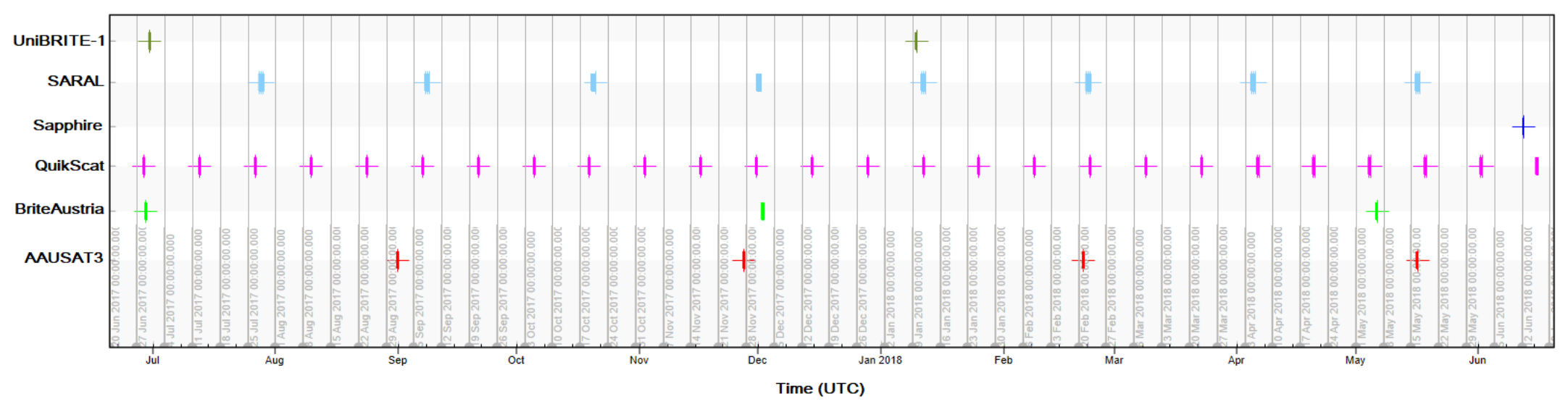

Figure 4.7: Close Approaches with NEOSSat 


\subsubsection{Observation Schedule}

Eight observation events took place on six unique satellites over the course of a one year period from 29 June 2017 until 12 June 2018.

Table 4.3: Observation Events

\begin{tabular}{ccccc}
\hline \hline Event \# & Object & Year & Day of Year & Date \\
\hline \hline 1 & BriteAustria & 2017 & 180 & 29 June \\
2 & UniBRITE-1 & 2017 & 181 & 30 June \\
3 & AAUSAT3 & 2017 & 243 & 31 August \\
4 & SARAL & 2017 & 251 & 8 September \\
5 & AAUSAT3 & 2017 & 331 & 27 November \\
6 & BriteAustria & 2017 & 335 & 1 December \\
7 & QuikScat & 2018 & 152 & 1 June \\
8 & Sapphire & 2018 & 163 & 12 June \\
\hline \hline
\end{tabular}

\subsection{Data Processing}

The proposed data flow guiding the acquisition, processing, and exploitation of the data from NEOSSat can be visualized in Fig. 4.8.
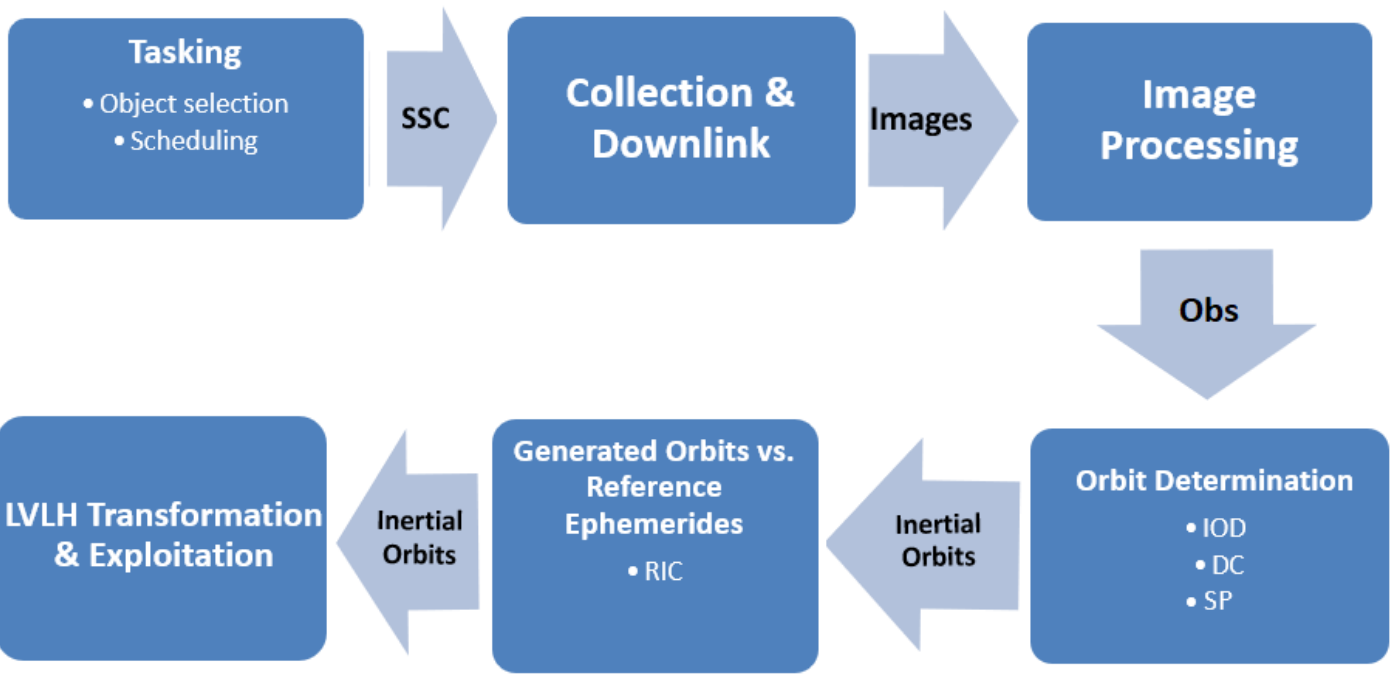

Figure 4.8: Data Flow for observation campaign 


\subsubsection{NEOSSat Tasking}

NEOSSat is constrained in its operations with an exclusion cone of $45^{\circ}$ from the Sun, an exlusion cone of $10^{\circ}$ from bright celestial objects such as the Moon, and a minimum Earth limb angle of $10^{\circ}$. These constraints are designed to prevent damage to sensitive spacecraft equipment, or prevent the loss of fine pointing control during imaging. Although the satellite may have a direct LOS to the target, it is important that imaging be undertaken when NEOSSat has a vantage point which allows a sufficient amount of light to be reflected off the target in order for the CCDs to measure a sufficient response. In addition to all of these requirements, the target must be imaged while background stars are visible in order to allow for matching to a star catalog to determine the pointing RA and DEC.

NEOSSat was designed with a $60 \mathrm{arcsec} / \mathrm{sec}$ slew rate limitation. However, recent operational changes have increased that limit and the satellite operated with a 90 $\operatorname{arcsec} / \mathrm{sec}$ limit for the taskings undertaken in this campaign. If NEOSSat is unable to sufficiently match the relative angular velocity of the target RSO, the resulting point source (See Fig. 2.5) of the RSO in the image will become elongated or blurred and result in a greater uncertainty in the observed position. NEOSSat is designed to operate with a constant slew rate during tracking; this time duration is normally for the series of 4-6 images being taken, representing a span of two to three minutes. Over an observation interval, as seen in Fig. 4.9, the relative angular velocity of the target RSO is not constant, and NEOSSat is tasked to slew at an averaged slew rate for the interval, leading to the earlier or later images potentially having some elongation or blurring of the RSO point source, dependent on the rate mis-match.

For NEOSSat taskings on objects in either MEO or higher orbits, the satellite has the ability to slew between the wide variety of targets with ease, this is due to the large number of objects in view with low relative angular velocities due to their large semimajor axes. Due to the unique tasking on objects in a similar LEO orbit, NEOSSat was only able to task in certain sections of the orbit in order to ensure the satellite was both pointing in an anti-solar direction, as well as having a sufficiently low relative angular velocity. This type of configuration and resulting imaging opportunities can be seen in Figs. 4.10 and 4.11. The overall period of 


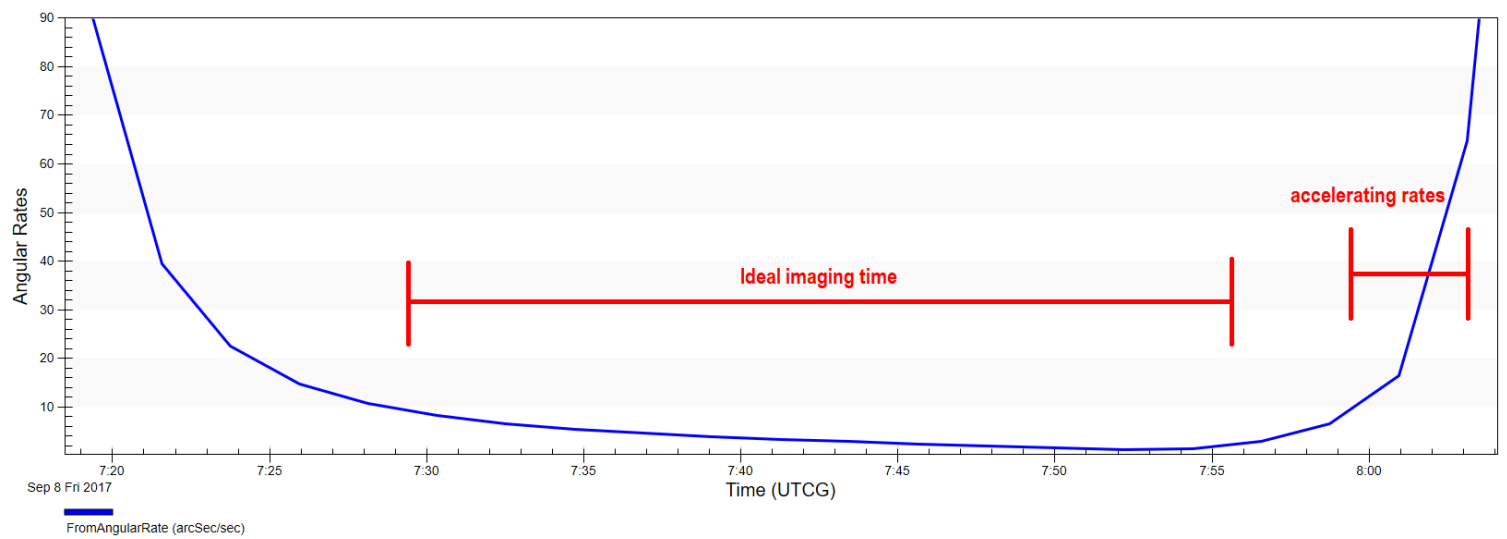

Figure 4.9: SARAL's Relative Angular Velocity relative to NEOSSat during an observation interval in $\operatorname{arcsec} / \mathrm{sec}$

time in which an object is viewable by NEOSSat is referred to as an event, while an individual viewing opportunity within an event is referred to as an interval, as seen in Fig. 4.11. This orbit configuration posed the possibility that the resulting orbit quality might be of concern due to the continual observation of the satellite in one particular portion of its orbit.

Tasking of NEOSSat was handled by the HEOSS team at DRDC in communication with the author. Once specific events were selected, the HEOSS team balanced system requirements in an attempt to gather as many observations as possible on the target satellite. The primary goal of the observation campaign was to gather a sufficient number of observations to determine if it was possible to generate an orbit for the observed satellite.

Once uploaded to the satellite on a scheduled pass, NEOSSat executed the tasking in a sequential fashion in accordance with the SSC tasking file. The images were subsequently downloaded by the CSA, which passed the files along to the HEOSS science team for data processing.

\subsubsection{Image Processing}

Once in possession of the images and NEOSSat GPS data, the HEOSS science team processes the imagery on the SQUID3 image processing system [17]. This system performs noise filtering for raw images, identifying background star streaks, centroiding 


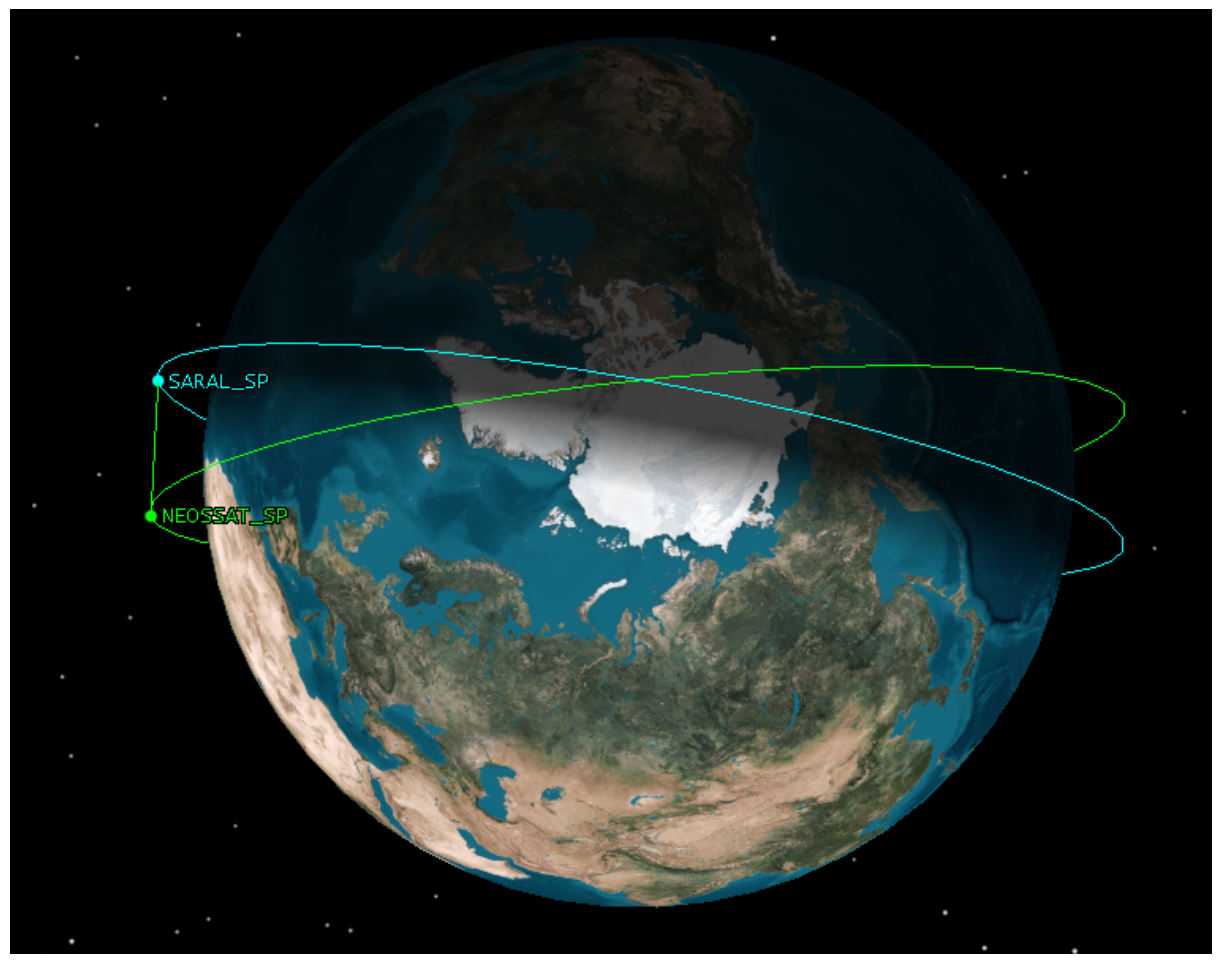

Figure 4.10: Predicted NEOSSat Orbit while observing SARAL on 8 Sep 2017.

and correlating star streaks to a star catalog, allowing the identification of baseline RA and DEC information for the images. From this information, the centroided objects in the imagery could be identified, their angular positions determined, and most importantly, correlated to the appropriate RSO. In standard NEOSSat tasking, correlation becomes an important task in order to distinguish one object from another, for close proximity events, the object can become incredibly bright, making it trivial to identify, although unfortunately, this brightness can saturate NEOSSat's CCD, resulting in more uncertainty with the calculated angular position of the object in the centroiding process.

Once processed by the NEOSSat science team, metric observations were transmitted in a SSN standardized format called B3 Type 9. Type 9 can be used for a space-based observing platform. B3s comes in internal and transmit formats, both of which includes the sensor identification number, target SSN ID, time of observation, RA and DEC of the target in the J2000 reference frame, as well as the ECEF 


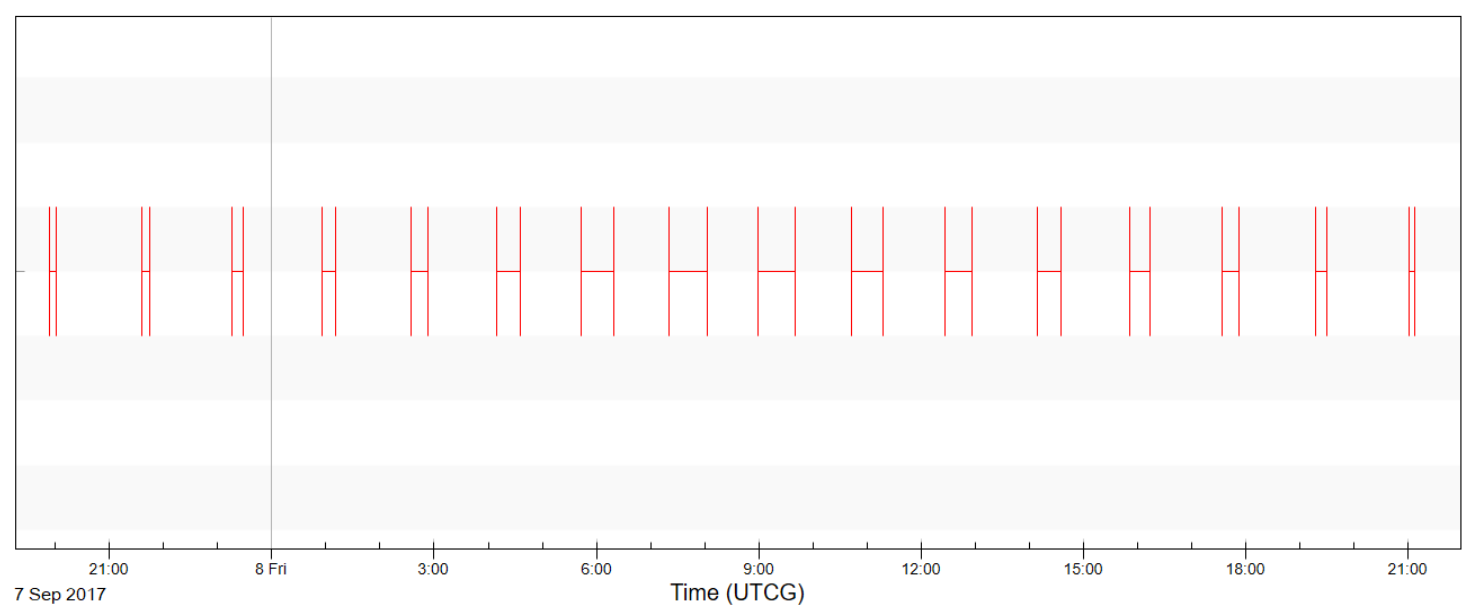

Figure 4.11: Predicted NEOSSat observing intervals during the SARAL event on 7 and 8 Sep 2017.

position of the observing platform ${ }^{13}$. Fortunately, when adding a Windows Script Component to AGI's ODTK, it is able to utilize the transmit formatted data directly.

\subsubsection{Reference Ephemerides}

In order to provide a reference comparison for the produced orbits of NEOSSat and the target satellite, reasonable sources of truth data had to be identified. One source of position information that could be identified was NEOSSat's ephemerides. These ephemerides come in two forms, the first are predicted ephemerides generated by the CSA's SatOps team. These predicted ephemerides are produced on a weekly basis for a seven-day period into the future and are seeded by the latest GPS positions downlinked from NEOSSat. The second form of ephemerides are available post-event when downlinked from NEOSSat after the observing time period. Although not directly useful for future planning, the post-event ephemerides can be used to seed future predicted ephemerides as well as being utilized as references for past observations. It is important to note that the post-event ephemerides are more accurate than the predicted ephemerides as the only errors in the measurements are from the GPS receivers themselves, there is no induced errors from the propagator. NEOSSat's post-event

\footnotetext{
${ }^{13}$ AGI, ODTK Measurement Formats, 2018, (Accessed Jul 4, 2018),
} http://help.agi.com/odtk/index.html 
ephemerides have a 3D 3-sigma one meter position accuracy [31]. These ephemerides were utilized as the position truth information of NEOSSat for the experiment, while the satellite ECEF position, generated live by the onboard GPS receivers, in the B3 observations were used as truth information for the orbit determination process. A comparison of the accuracy of publicly available TLEs to NEOSSat GPS ephemerides can be seen in Fig. 4.12.

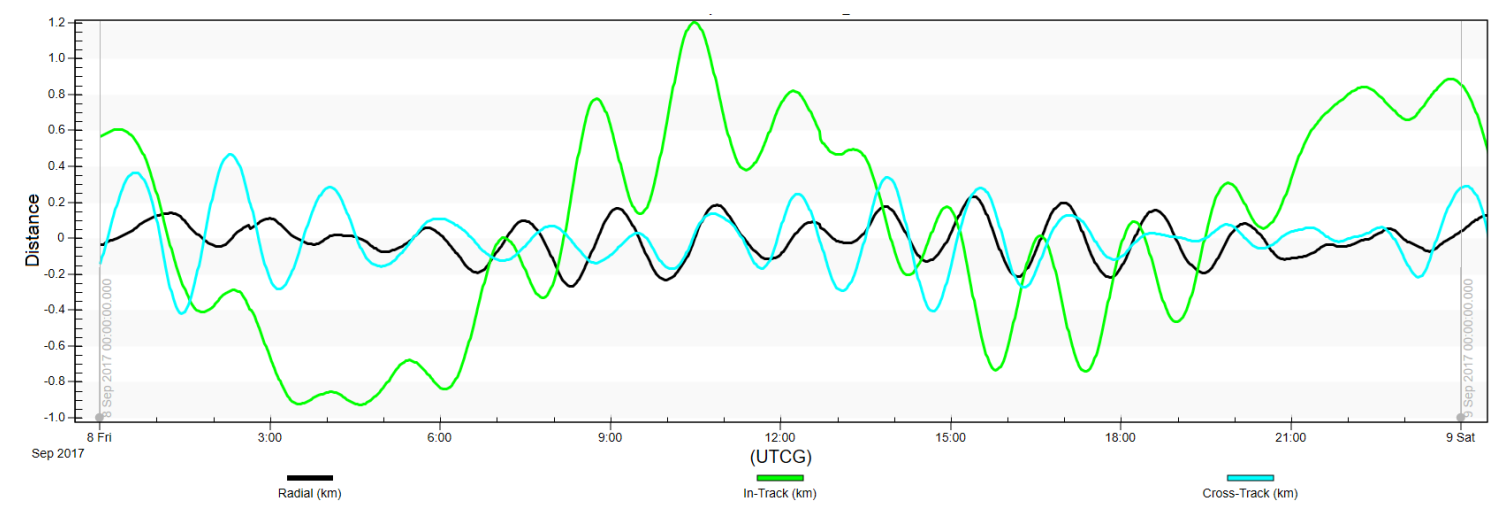

Figure 4.12: NEOSSat Ephemerides compared to available TLE

As previously mentioned in Section 3.5, SARAL's post-event ephemerides were identified as a source of reference calibration information for one of the targets. With a one centimeter radial and cross-track, as well as five centimeter in-track position accuracy, this data was the most accurate information which could be identified. Unfortunately, the ILRS does not produce ephemeris data for any of the other satellites which were imaged by NEOSSat, and therefore another source of truth data had to be identified.

Fortunately, the final source of position information that could be identified was furnished by the USAF's 18th Space Control Squadron (18 SPCS) and the United States Strategic Command (USSTRATCOM). 18 SPCS is the unit responsible for maintaining the SSN's space catalog${ }^{14}$. In addition to the publicly available TLEs used to describe the orbit of various objects, 18 SPCS generates high-precision SP ephemerides of all RSOs tracked by the SSN. This data is used by the HEOSS science team as part of DND, in order to optimize the tasking accuracy for NEOSSat. This

\footnotetext{
${ }^{14}$ Peterson AFB, 18SPCS, 2017, (Accessed Jun 26, 2018), http://www.peterson.af.mil/About/Fact-Sheets/Display/Article/1060346/18th-space-controlsquadron/
} 
information represents the most accurate position information available for the entire group of observed satellites. Unfortunately, the accuracy of the data is not published, and in accordance with the author's agreement with USSTRATCOM, no comparison of the accuracy of this data will be published. However, defence scientists at DRDC, as well as the author can attest that the data is significantly more accurate than publicly available TLEs and was treated as truth position data for the remaining observed satellites. However, SP ephemerides were not used for the metric accuracy assessment of the sensor. A comparison of the accuracy of publicly available TLEs to SARAL ILRS data is shown in Fig. 4.13.

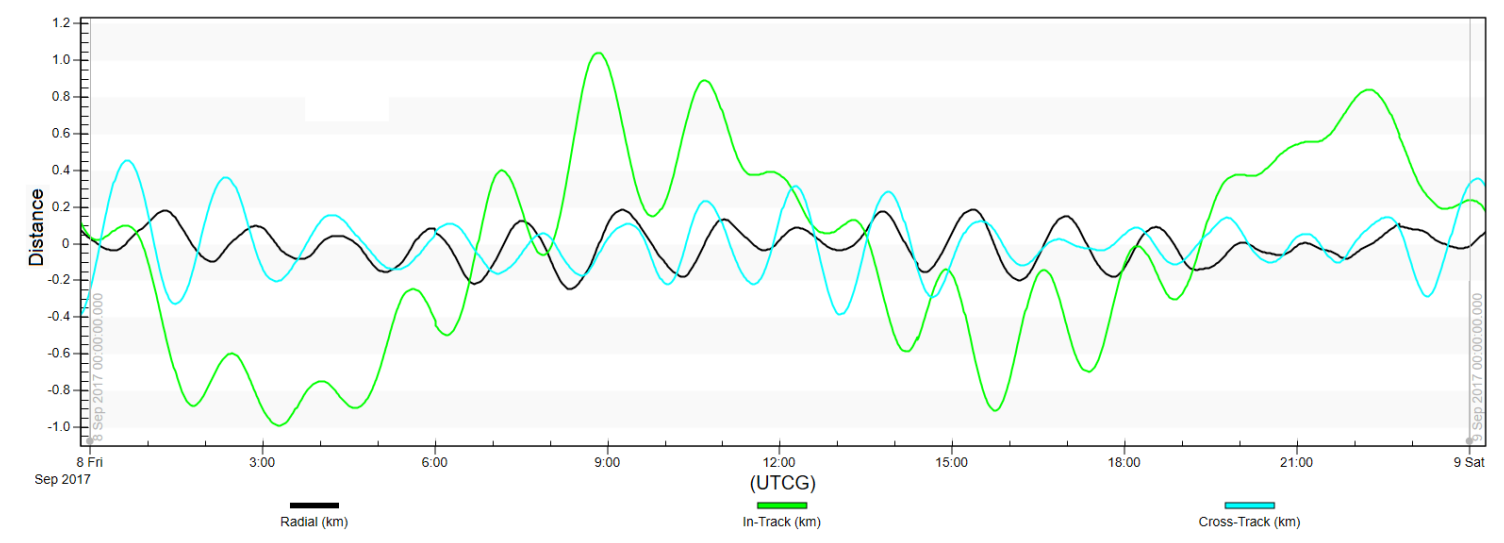

Figure 4.13: SARAL Ephemerides compared to available TLE

\subsubsection{ODTK Orbit Determination}

In ODTK, the orbit determination process follows a methodology nearly identical to that outlined in Section 3.2 which allows for each subsequent step to further refine the orbit estimate. The process begins with a coarse orbit approximation using Initial Orbit Determination (IOD), followed by the use of Least Squares (LS) refinement, finishing with Optimal Orbit Determination (OOD).

$$
I O D \Rightarrow L S \Rightarrow O O D
$$

IOD in ODTK for range-less observation data follows the Gooding Angles only algorithm. If it is possible for the program to find a solution for the velocity of the object at $r_{1}$ that satisfies the conditions of $r_{2}$, then the algorithm will converge to a 
local solution for the orbit. This solution is a very coarse approximation of the orbit and only useful as a seed for further processing. There are a variety of reasons of why this process may not work, primarily due to the geometry and timing of observations. Due to the orbital configuration of the observation campaigns, it was possible that observability may become an issue for the algorithm as well as manually picking observations which were sufficiently spaced. If NEOSSat was unable to generate an orbit from one interval of observations, then a subsequent interval would need to be used to generate the IOD, leading to the possibility of multiple orbit solutions.

The LS process in ODTK performs a series of differential corrections identical to the DC process mentioned in Section 3.2. The LS process requires a user to select a series of measurements over a certain time span to fit the orbit, or even to specifiy different spans, each of which would allow for separate LS processes to fit an orbit. Ideally the values of the estimated orbit solution will begin to converge with each subsequent iteration towards the optimal state vector. If the orbit were to diverge, either a different series of observations would need to be used, or perhaps the chosen IOD seed was incorrect, a particular problem when multiple revolutions were used to create the initial state estimate. An output of this process is the covariance required to implement the filtering process in OOD.

The final portion of orbit determination in ODTK is OOD. OOD employs two major methods: filters and smoothers. Filters are EKFs running forwards in time, while smoothers run backwards in time and work to smooth the results from piecewise filters. Filters are used to refine the orbit estimate and can be easily contrasted to the LS process. While LS uses all of the measurements in a single LS iteration to determine an orbit estimate, a filter will incorporate a single measurement at a time instant, and when considering the covariance of the object at that time, will either reject the observation for being outside a certain error tolerance, or will use the measurement in order to refine the orbit state and covariance estimate. The filter will then take the new orbit state and covariance estimate and propagate until the next observation. At that time, the new measurement is incorporated and the orbit estimate is refined once again. This process is repeated until no more measurements are available. 
The use of the different capabilities in ODTK were carried out in accordance with the methodologies outlined by the author in Section 3.

\subsubsection{Generated Orbits vs. Reference Ephemerides}

In order to assess the accuracy of the resulting orbits, all generated orbits were compared to their respective truth ephemerides in order to assess the position accuracy of the estimates. This comparison is made using STK by exporting the generated orbits in a STK ephemeris format and subsequently comparing the difference in their Radial, In-track, and Cross-track components (RIC).

\subsubsection{LVLH Transformation \& Exploitation}

Once the generated orbits are exported to STK for comparison and visualization, they can then be exploited for purposes related to proximity operations. Within STK, the orbits are transformed into the LVLH representation where the observed satellite is the target spacecraft and NEOSSat is the chaser spacecraft. The generated positions are exported into a comma separated value (csv) file which is then ingested into MATLAB for visualization. Similar to the formulation outlined in Section 2.4, the STK LVLH reference frame is defined as: $\vec{L}_{x}$ along the position vector, $\vec{L}_{z}$ along the orbit normal, and $\vec{L}_{y}$ towards velocity, completing the right-handed system of $\vec{L}_{y}=\vec{L}_{z} \times \vec{L}_{x}$

\subsection{Error Characterization}

There are a variety of possible error sources in the data processing chain. They can be primarily divided into data source, observation, and data processing errors. This section will attempt to estimate the predicted position error of the observations. While this process is an approximation, the performance of metric calibration on observations, as outlined in Section 5.2, has the intent to measure the true metric error in the sensor for Section 4.6.2. 


\subsubsection{Data Source Errors}

Data source errors are primarily focussed on the errors associated with the highprecision ephemerides acquired by the author.

For the ILRS ephemerides furnished by the ILRS, the ephemerides themselves have been published to be as accurate as one centimeter in the radial and cross-track directions, while being five centimeter accurate for the in-track directions [30]. This data is the most accurate available source obtained by the author and is one to two orders of magnitude smaller than the physical size of SARAL itself. This comparison in the size of error to the size of the object, effectively renders the associated error to be negligible.

The GPS accuracy of NEOSSat is published to have a 3D 3-sigma theshold of sub-meter accuracy [31]. The dual GPS receivers measure the GPS position to the center of the imaging focal plane of the CCD. When assuming a worst case one meter offset from the true position while observing an object at a nominal $100 \mathrm{~km}$ distance, this position error would result in a two arcsecond metric error from the actual true position. Although smaller than the three arcsecond scale of an individual pixel on the CCD, this value is not negligible. This process can be extrapolated even further for some of the very close proximity observations at a nominal range of $10 \mathrm{~km}$, resulting in a 20 arcsecond metric, or 7-pixel, error.

Unfortunately, the associated error on the SP ephemerides could not be published. What can be said is that these ephemerides are significantly more accurate than the publicly available TLEs and are considered exact for the purposes of this thesis.

\subsubsection{Observation Errors}

The observation errors of NEOSSat have been previously characterized [17] and are primarily focussed on issues with timing, centroiding, observation corruption, and observation format precision.

NEOSSat observation timings for the CCD are read out to the millisecond. Provided NEOSSat tracked objects with slew rates of up to $90 \mathrm{arcsec} / \mathrm{sec}$ for this observation campaign, this 1 millisecond error could potentially result in up to 0.09 arcsecond metric errors. 
Centroiding errors are significant and directly tied to the size of the RSO signal in an image. If there is a slew rate mis-match, this value will scale according to the number of pixels the RSO signature is spread over. There is a deliberate effort to minimize this mis-match by specifically selecting imaging opportunities that avoid large differences in start and stop slew rates, as well as modifying exposures times in order to minimize RSO size. Nevertheless, this process is not perfect and errors can be caused due to mis-matches. As an example, a five arcsec/sec slew rate mismatch for a bright object in a two second image will have a centroid spanning 10 arcseconds, or three pixels.

Observations typically suffer some form of corruption prior to being processed by the SQUID3 image processor. In the process of taking an image, the CCD can encounter cosmic ray particles, which are picked up just like any photo electron registered by the detector array. If one of these cosmic ray hits are in proximity to an object measurement, this signal may be included and can broaden the source signal, potentially moving the calculated center of the centroid away from the actual center of the source signal. SQUID3 attempts to minimize these induced errors but is not always able to do so.

The B3 observation format output by SQUID3 is only able to store position information to a certain accuracy. This leads to a possible truncation error for the RA or DEC measurements to a worst case scenario of 0.75 arcseconds in RA, and 0.18 arcseconds in DEC.

\subsubsection{Data Processing Errors}

Errors in the orbit fitting process are characterized by the covariance matrix of the resulting DC or filter as per Section 3.2.6. The diagonal elements of these matrices outline the variance of the measurements with respect to the resulting orbit fit in order to describe the probability distribution of the object being off of its exact trajectory. 


\section{Chapter 5}

\section{Experiment Results}

\section{$5.1 \quad$ Introduction}

This chapter details the experimental results from the metric assessment performed on SARAL, the application of the two different OD methodologies proposed in Section

3 , as well as the results from converting the generated orbits to the LVLH reference frame for exploitation in proximity operations. The OD methodologies were applied to each of the 8 observation events outlined in Section 4.4.1.

\subsection{Metric Assessment}

From the observation event with SARAL on 8 Sep 17, 60 observations, each with one RA and one DEC measurement, were utilized in order to perform an initial characterization of the noise from NEOSSat observations in the LEO to LEO operations range. The measurements for this event were all in $2 \times 2$ binning of the CCD and the results can be seen in Figs. 5.1 and 5.2. 


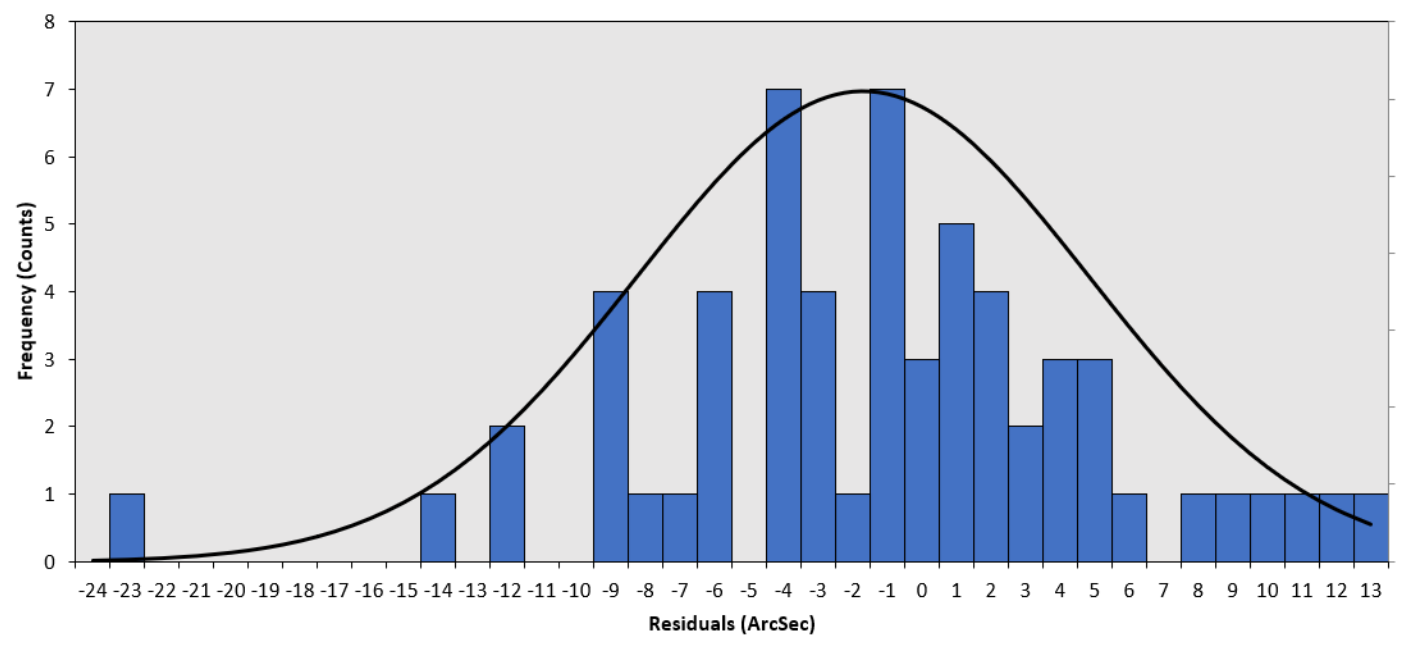

Figure 5.1: Right Ascension Residuals

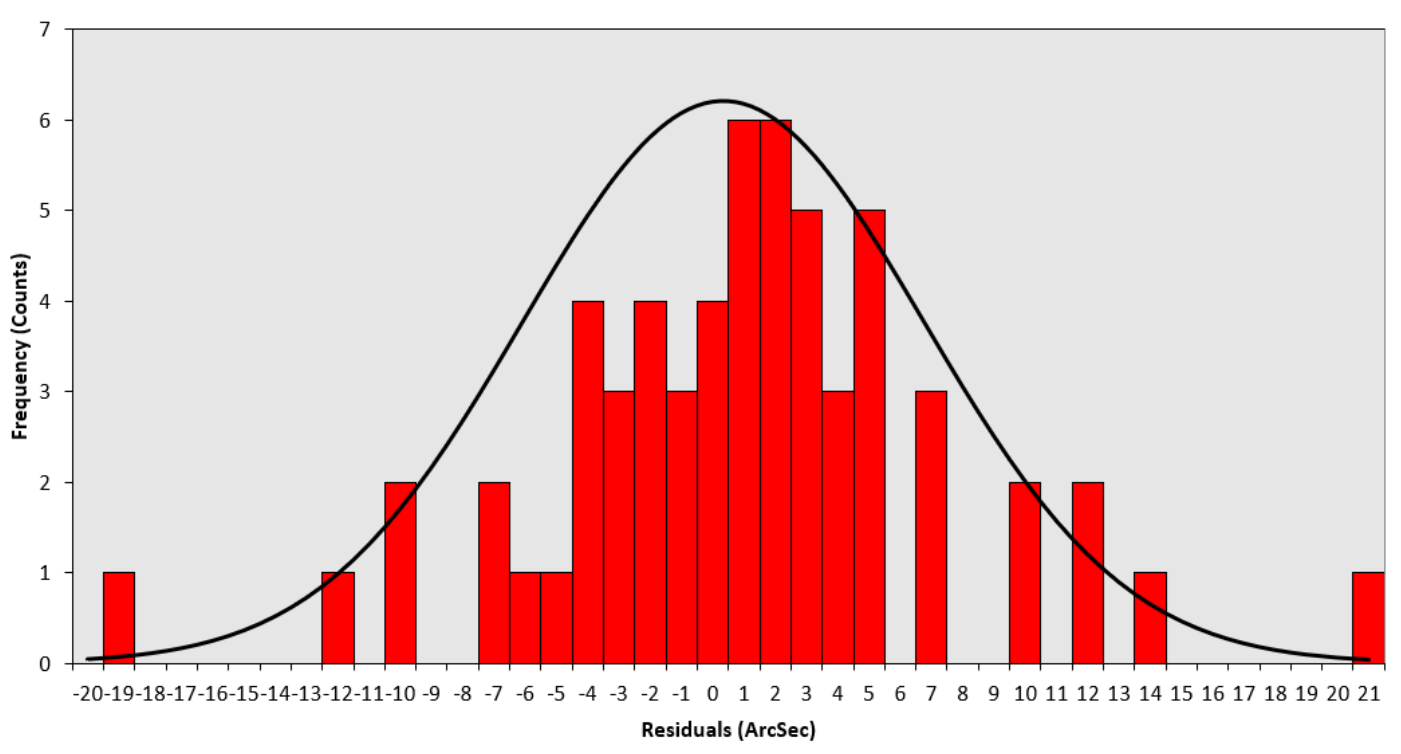

Figure 5.2: Declination Residuals 
Although a small sample size, the distribution of these results do appear somewhat Gaussian in nature. In taking this assumption, the results can be characterized in Table 5.1.

Table 5.1: Metric Assessment Results (values in arcseconds)

\begin{tabular}{ccc}
\hline \hline & RA & DEC \\
Binning & $2 \times 2$ & $2 \times 2$ \\
\hline \hline$\sigma$ & 6.54 & 6.43 \\
Bias & -1.70 & 0.35 \\
\hline
\end{tabular}

These results vary significantly from the GPS campaign metric results outlined in Table 2.1. To begin, the standard deviation of the results, $\sigma$, is approximately twice the magnitude, while the biases also vary significantly. There are a variety of possible reasons for these discrepancies such as consistently imaging the object at one portion of its orbit, as well as the oversaturation of pixels in the CCD images themselves. Within the SQUID3 image processor, the chosen central position of the object in the image is the result of a mathematical average of the position and the intensity of different pixels containing the source signal. This makes the processor more susceptable to errors when processing oversaturated images, particularly ones that are not point-source shaped. The elongated RSO signals provide more uncertainty in the object position. The contrast in tight and saturated RSO signals can be seen in Figs. 5.3 and 5.4 . 


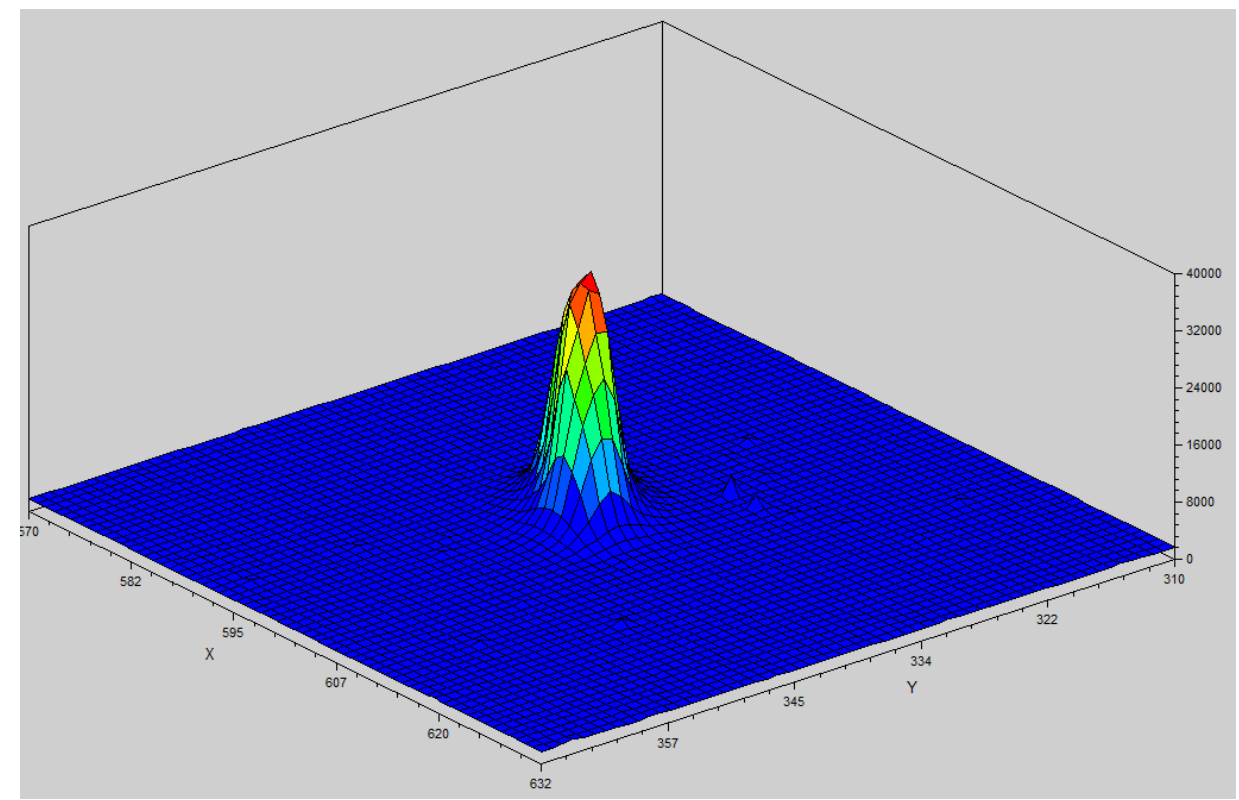

Figure 5.3: Tight RSO Signal

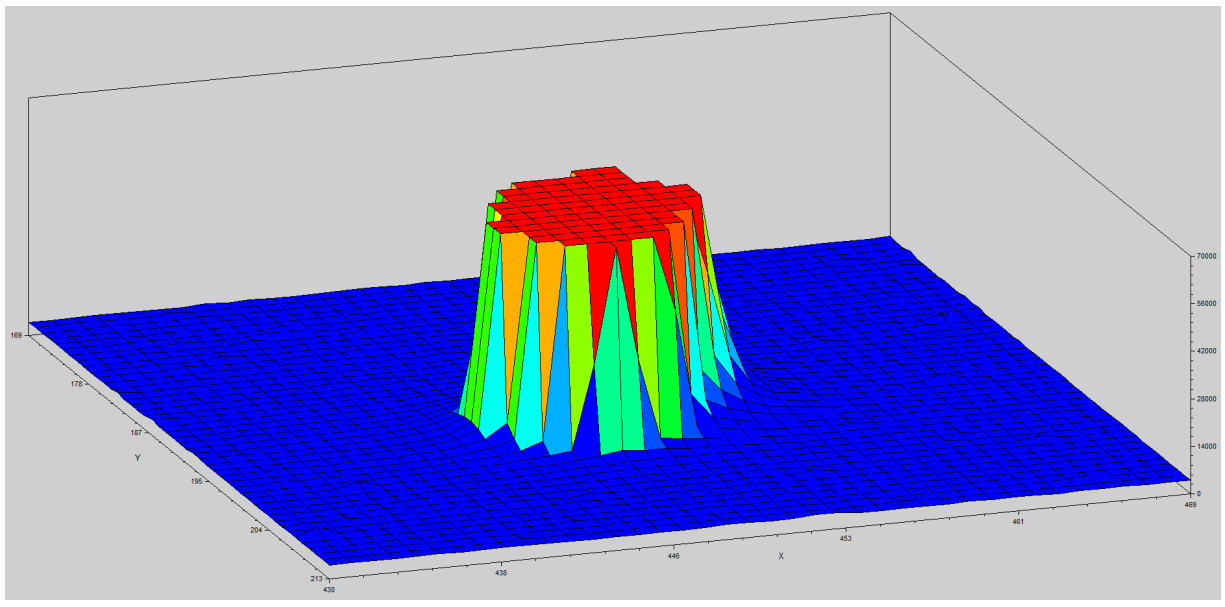

Figure 5.4: Saturated RSO Signal 
In addition to these possible causes is the fact that only 60 observations were taken from a true reference orbit in this campaign and this number is only a third of the 183 used in the GPS campaign. If more observations were to be taken of SARAL, the actual noise could likely be better characterized with greater certainty. The primary intent of this metric assessment was not to provide an in-depth assessment but to properly setup the noise characteristics of the EKFs used in LEO to LEO operations for the orbit determination processes of Methods 1 and 2. 


\subsection{BriteAustria (1)}

The first proximity event with BriteAustria had 6 intervals which allowed sub-90 arcsec/sec angular rate tracks with NEOSSat. The first interval started at 01:19:00 UTC on 29 Jun 2017 and the final interval ended at 10:00:00 UTC the same day. Within these intervals, NEOSSat was able to track and collect 22 observations, each observation containing a pair of RA and DEC measurements. During this particular event, all 22 observations were collected in the $2^{\text {nd }}$ interval from 02:57:56 UTC until 03:27:03 UTC as seen in Fig. 5.5.

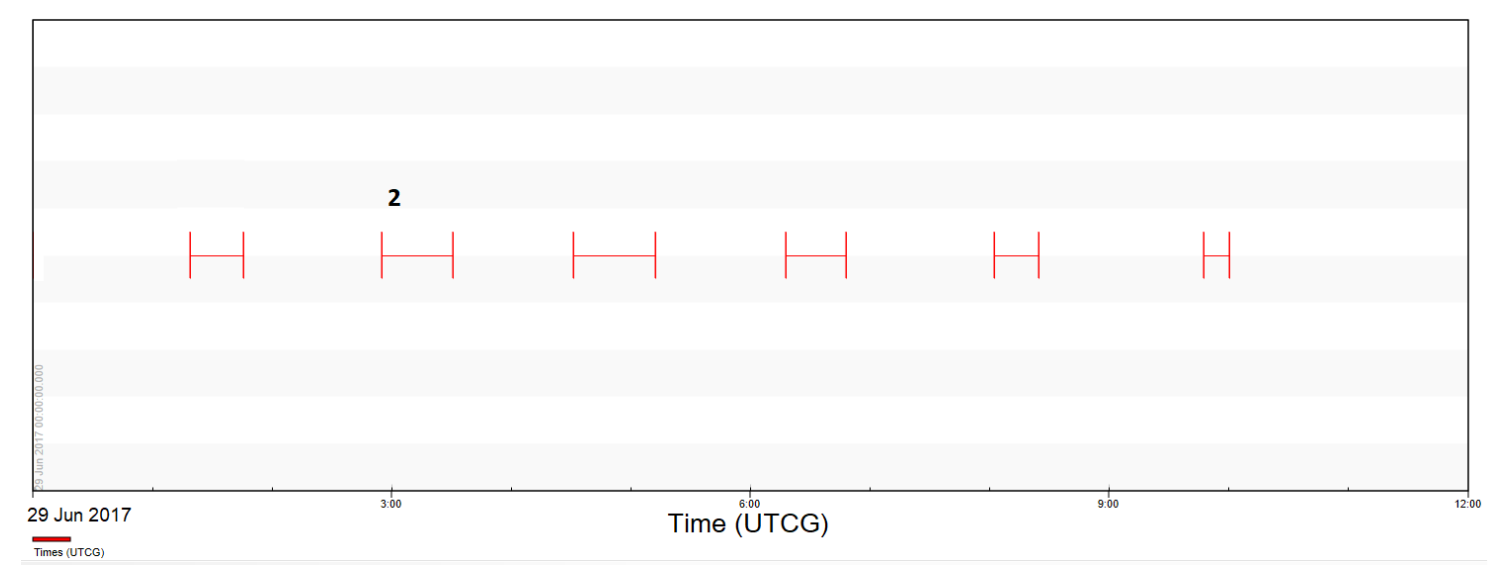

Figure 5.5: BriteAustria observation intervals from NEOSSat

\subsubsection{Method 1}

The observations within the single orbit revolution were sufficiently spaced to produce an IOD with ODTK. From this IOD, there were also sufficient observations to allow for a DC to converge and create a resulting orbit with a covariance matrix representing the uncertainty in the orbit estimate. Due to there being no subsequent observations in the following re-visits, no filter from method 1 was seeded. The resulting orbit was compared to the reference SP orbit and compared in RIC in Fig. 5.6.

When comparing the resulting orbit fit to the SP ephemeris values in RIC, it can be seen that the orbit has a great deal of accuracy in the radial component, yet lacks a corresponding accuracy in the cross-track component. In addition, there appears to be a slight mis-match for the in-track velocity as that component grows over time. 


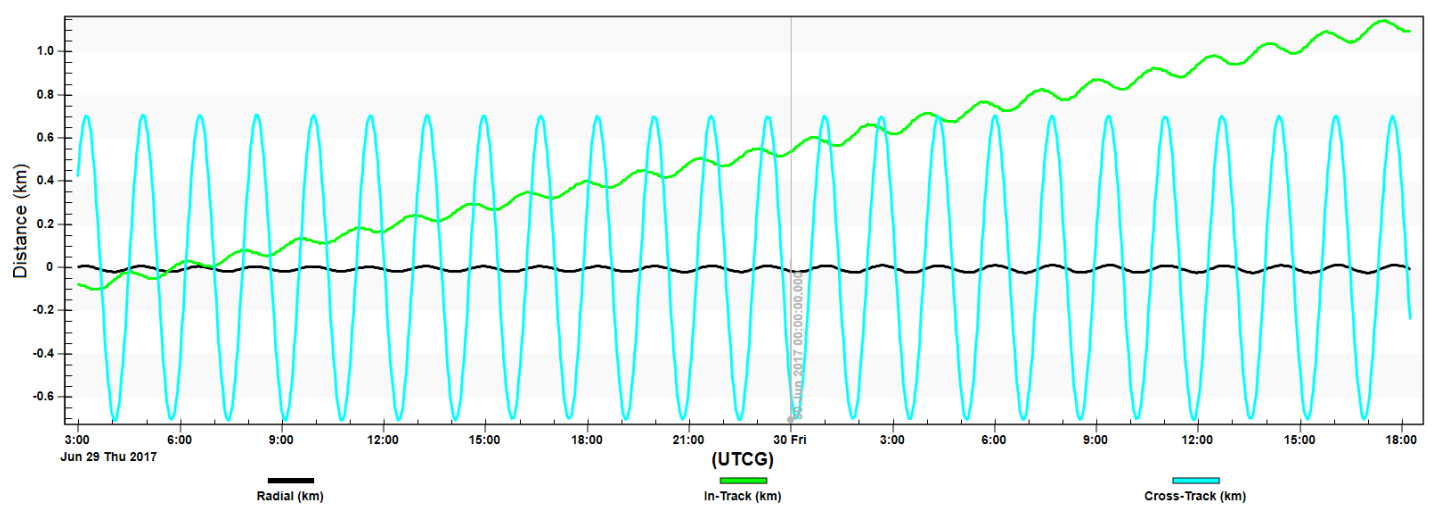

Figure 5.6: BriteAustria Opportunity \#1 Method 1 RIC Comparison

Over the course of the observations, the satellite varied in distance from 101-180 $\mathrm{km}$ with respect to NEOSSat. The 1- $\sigma$ confidence from the diagonal components in RIC of the LS covariance matrix are: R:10.6 m, I:131.9 m, C:869.8 m. These values describe a decreased confidence in both cross-track and in-track fits, while having a large confidence in the radial direction.

\subsubsection{Method 2}

The seeded orbit state for the satellite came from a TLE with an epoch of 19:40:33 UTC on 28 Jun 2017, approximately 8 hours before the observations and given estimated orbit uncertainties of R:102 m, I:471 m, C:126 m [16]. When running the filter with a rejection tolerance of 3- $\sigma$ (three times the characteristic noise found in the metric assessment), the majority of the observations were rejected. This was an interesting finding, particularly when compared to the results when the filter was re-ran with the rejection tolerance of 5- $\sigma$. The higher tolerance filter allowed all of the observations to be accepted. The RIC comparisons to the SP ephemerides for the two runs can be seen in Figs. 5.7 and 5.8 where discontinuities are due to the filter updating the position estimate upon addition of new observations.

Intuitively, increasing the rejection tolerance can be of concern as an increased number of poor observations are accepted. However in this case, it was an advantage as it appears the quality of the TLE and associated uncertainty were too restrictive. Including additional observations resulted in a vastly superior orbit estimate by allowing the additional observations to pull the estimate closer to the actual true orbit 


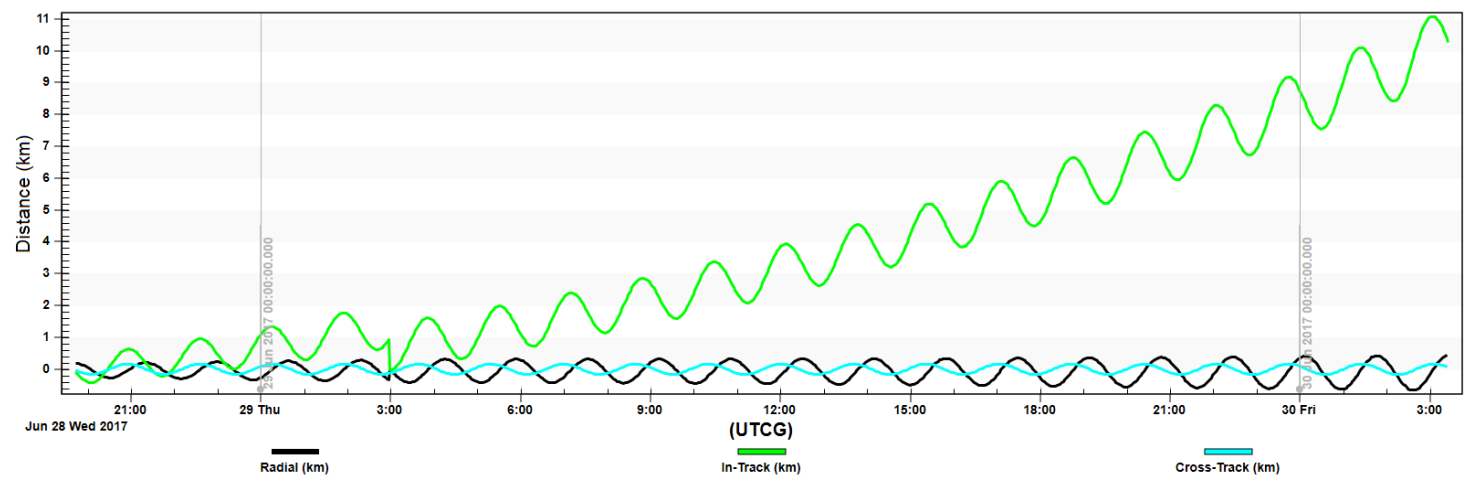

Figure 5.7: BriteAustria Opportunity \#1 Method 2, 3- $\sigma$ filter RIC Comparison

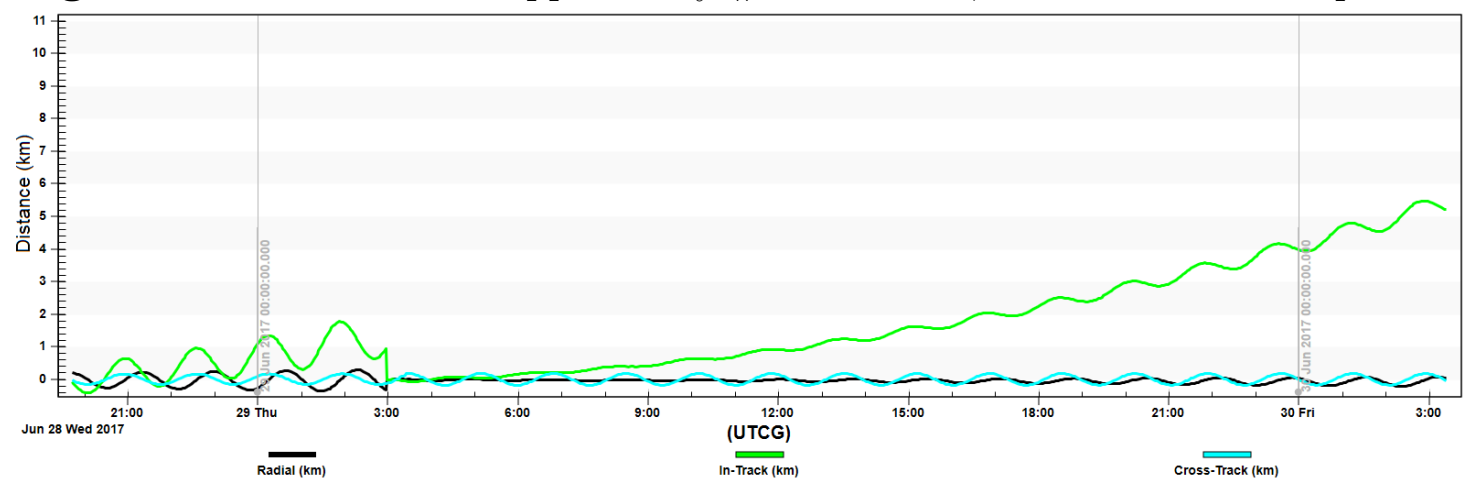

Figure 5.8: BriteAustria Opportunity \#1 Method 2, 5- $\sigma$ filter RIC Comparison

state. This migration can be is visualized in Fig. 5.9 where the 5- $\sigma$ orbit estimate was significantly more accurate.

The overall orbit estimate from Method 2 proved to be superior to that of Method 1 in the early period after the filter update primarily due to the a-priori confidence in the cross-track component. After approximately 2-3 hours of propagation, the mis-match of the in-track velocity for the Method 2 orbit outgrew that of Method 1. 


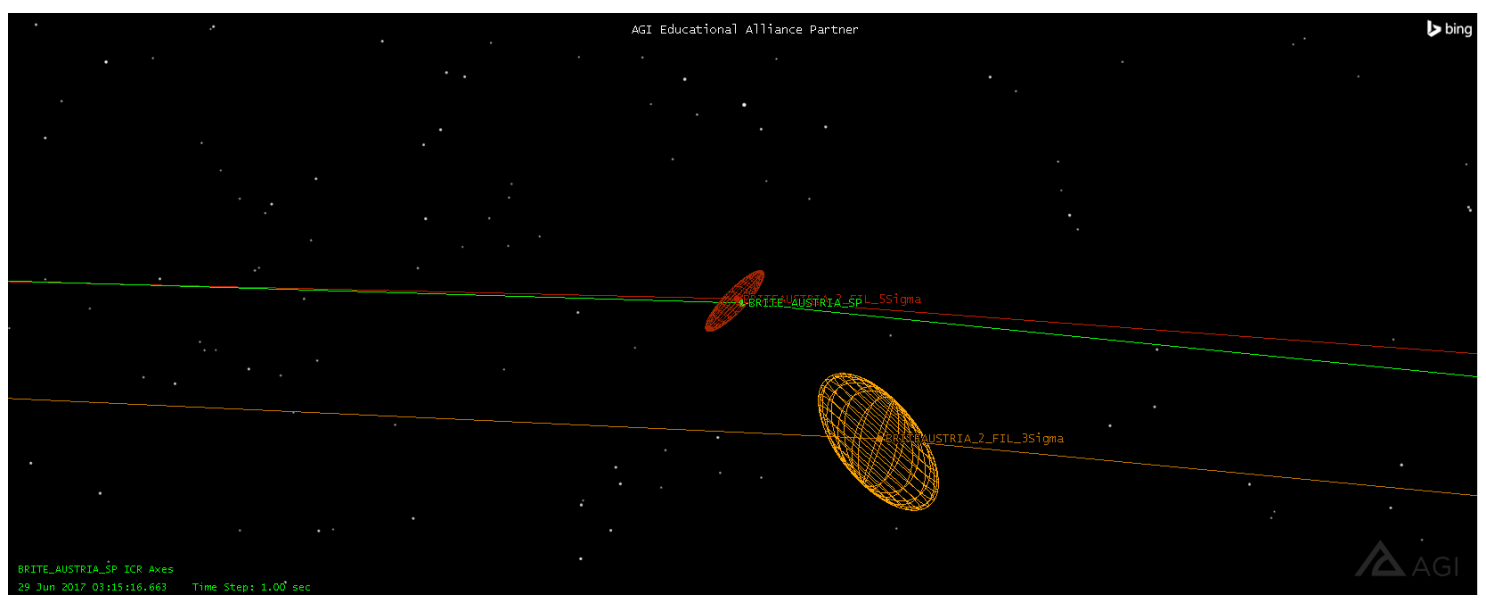

Figure 5.9: BriteAustria Opportunity \#1 Method 2, Orbit Estimates

\subsubsection{LVLH}

The generated orbit ephemerides for Methods 1 and 2 were exported from STK and were compared to the correpsonding SP ephemeris in LVLH in Fig. 5.10. The results are what was expected given the similar orbit estimates provided in Methods 1 and 2. The mis-match of the in-track velocity for Method 2 when compared to Method 1 is particularly evident in the third sub-plot of Fig. 5.10 with the orbit trace slowly growing as BriteAustria continues to drift past NEOSSat. A comparison of the error in the orbit fits can be seen in Fig. 5.11. This transformation preserves the relative position information and can be used to examine their relationships for exploitation. 
SP, Method 1, \& Method 2 Orbit in NEOSSat $\operatorname{LVLH}(X$ \& Y)

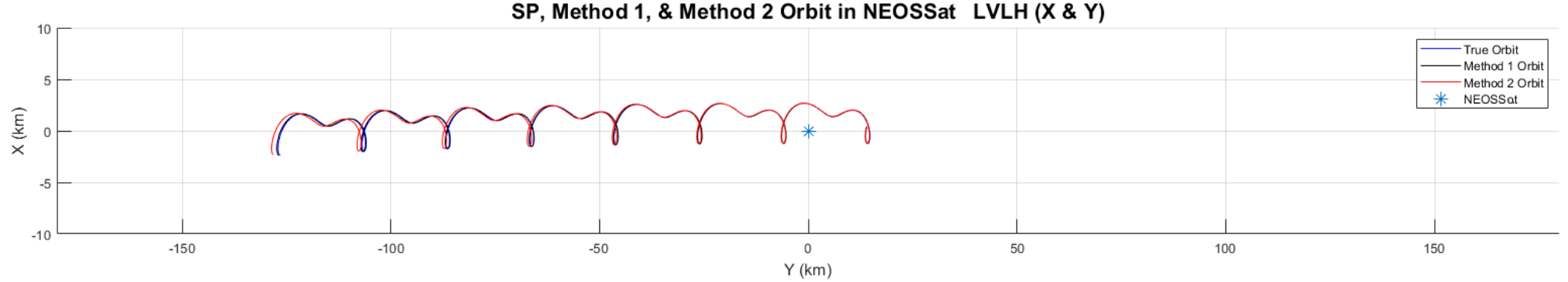

SP, Method 1, \& Method 2 Orbit in NEOSSat LVLH (X \& Z)

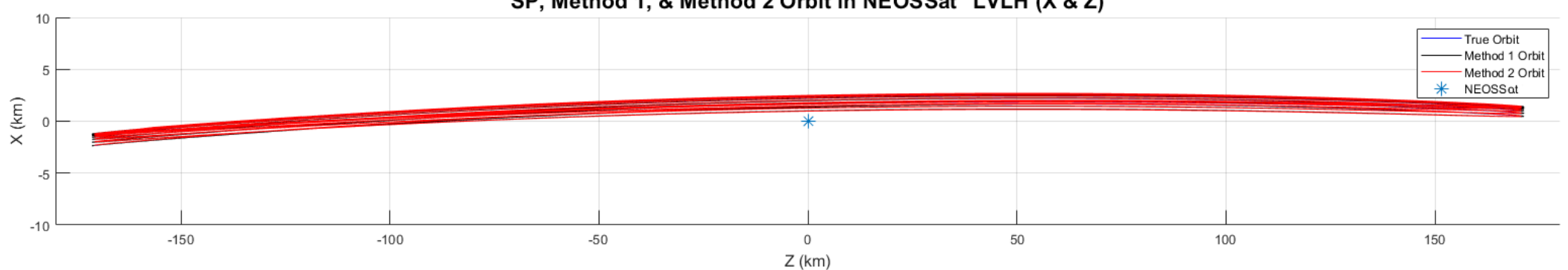

SP, Method 1, \& Method 2 Orbit in NEOSSat LVLH (Z \& Y)

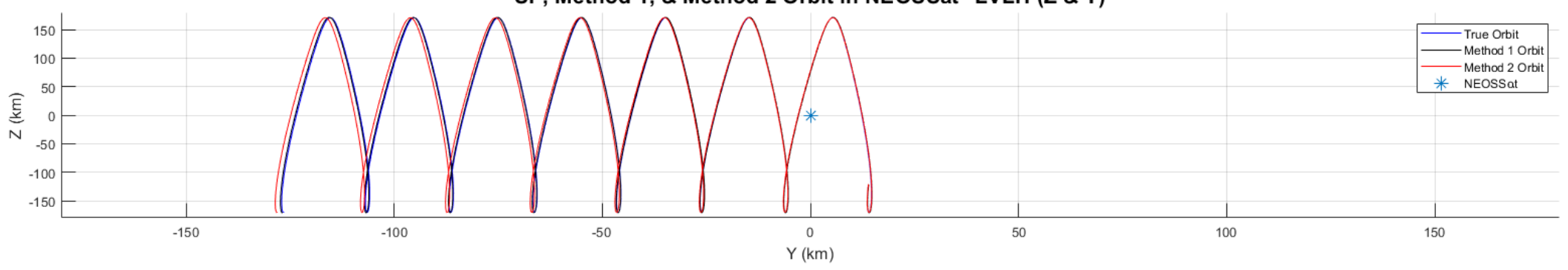

Figure 5.10: BriteAustria Opportunity \#1 LVLH 


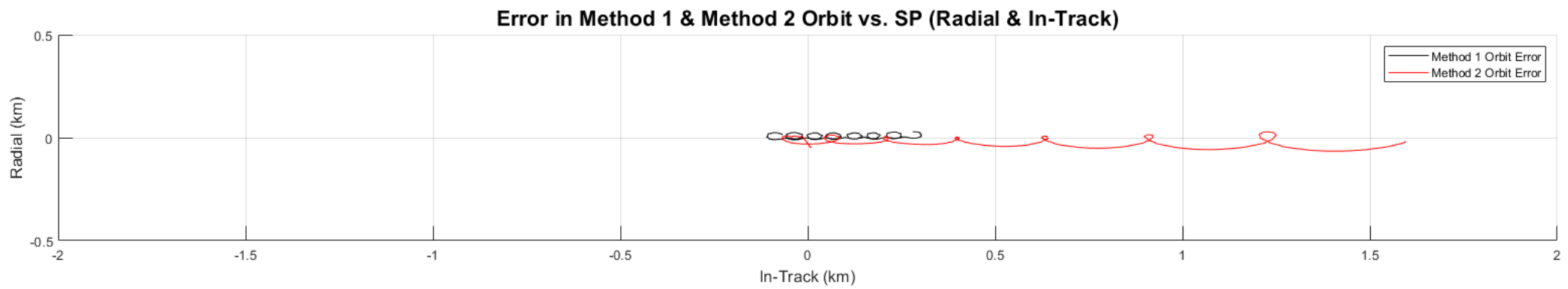

Error in Method 1 \& Method 2 Orbit vs. SP (Radial \& Cross-Track)

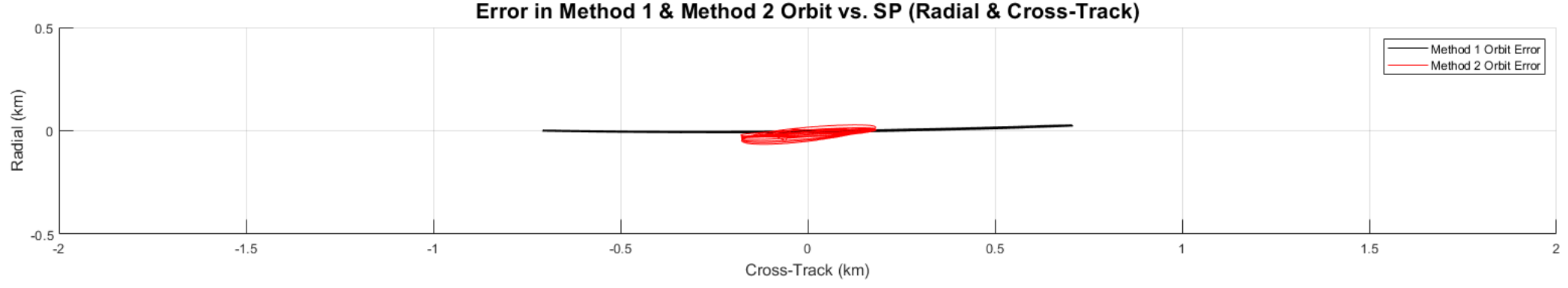

Error in Method 1 \& Method 2 Orbit vs. SP (Cross-Track \& In-Track)

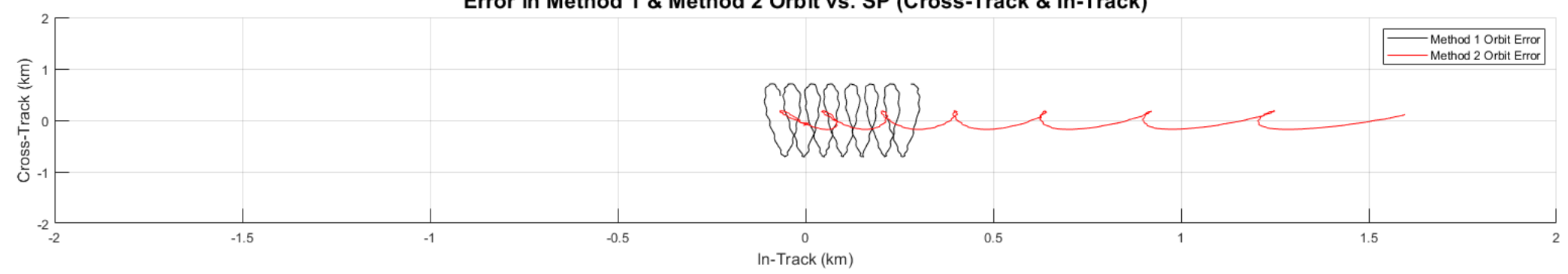

Figure 5.11: BriteAustria Opportunity \#1 Orbit Estimate Errors 


\subsection{UniBRITE-1}

The proximity event with UniBRITE-1 had 8 intervals which allowed sub-90 arcsec/sec angular rate tracks with NEOSSat. The first interval started at 00:54:45 UTC on 30 Jun 2017 and the final interval ended at 12:54:26 UTC the same day. Within these intervals, NEOSSat was able to track and collect 32 observations, each observation containing a pair of RA and DEC measurements. During this particular event, the observations were collected on the passes as seen in Fig. 5.12 and in accordance with Table 5.2.

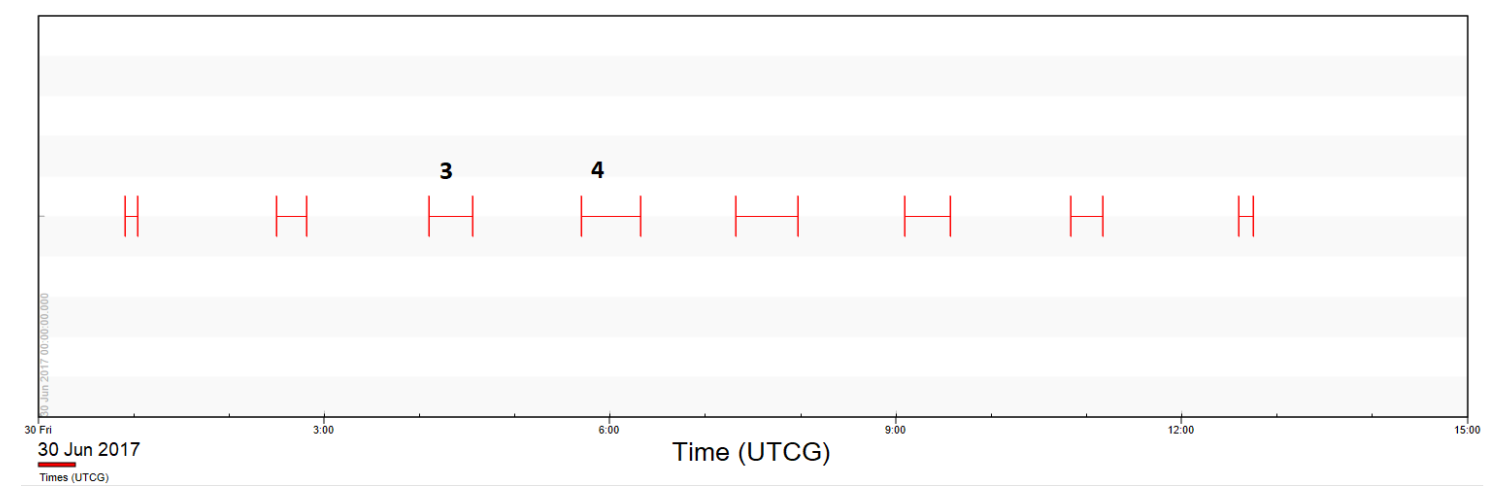

Figure 5.12: UniBRITE-1 observation intervals from NEOSSat

Table 5.2: UniBRITE-1 Observations

\begin{tabular}{ccccc}
\hline \hline Interval \# & Date & Start Time (UTC) & End Time (UTC) & \# of Observations \\
\hline \hline 3 & 30 Jun & $04: 10: 34$ & $04: 22: 03$ & 11 \\
4 & 30 Jun & $05: 44: 43$ & $06: 13: 44$ & 21 \\
\hline \hline
\end{tabular}

\subsubsection{Method 1}

The spacing of the observations within interval \#3 was insufficient to allow for an IOD, fortunately, the spacing of the observations within interval \#4 were sufficient. From this IOD, all measurements, including those from interval \#3 were sufficient to allow for a DC to converge and create a resulting orbit with a covariance matrix representing the uncertainty in the orbit estimate. Due to there being no subsequent observations in the following re-visits, no filter from Method 1 was seeded. The 
resulting orbit was compared to the reference SP orbit and compared in RIC in Fig. 5.13 .

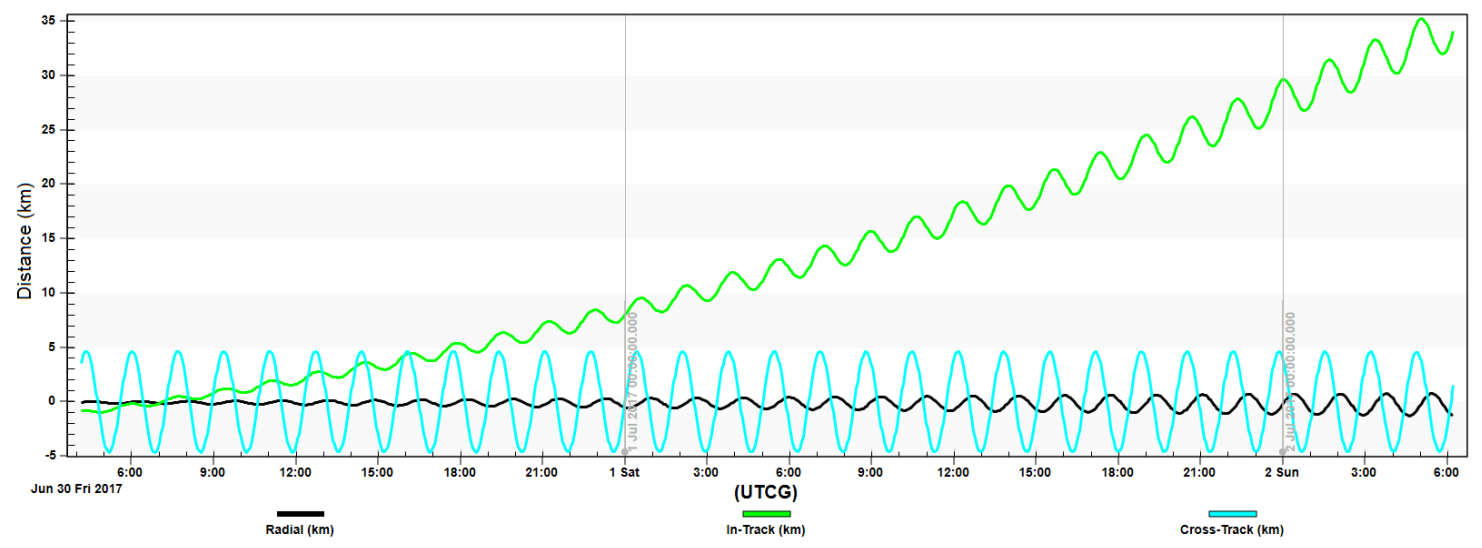

Figure 5.13: UniBRITE-1 Opportunity \#1 Method 1 RIC Comparison

From the resulting orbit fit, it can be seen that there was good accuracy in the radial component, yet a significant lack of accuracy in the cross-track component, and a significant mis-match of the in-track velocity as that component grows significantly over time. Over the course of the observations, the satellite varied in distance from 76$130 \mathrm{~km}$ with respect to NEOSSat. The 1- $\sigma$ confidence from the diagonal components in RIC of the LS covariance matrix are: R:98.7 m, I:1983.8 m, C:8459.7 m, reinforcing the significant lack of accuracy in the in-track and cross-track components with a significant lack of confidence in the orbit estimate.

This kind of orbit uncertainty fit would likely prompt a review of the orbit estimate by manually rejecting observations in order to tighten the resulting covariance matrix. This is an intensive process and not necessarily intuitive and easy to program for an automated system.

\subsubsection{Method 2}

The seeded orbit state for the satellite came from a TLE with an epoch of 12:25:13 UTC on 29 Jun 2017, approximately 16 hours before the observations and given estimated orbit uncertainties of R:102 m, I:471 m, C:126 m [16]. When running the filter with a rejection tolerance of $3-\sigma$, the observations from interval \#3 were all accepted, while rejecting those from interval \#4. When re-running the filter with the 
rejection tolerance of $5-\sigma$, all observations were accepted.

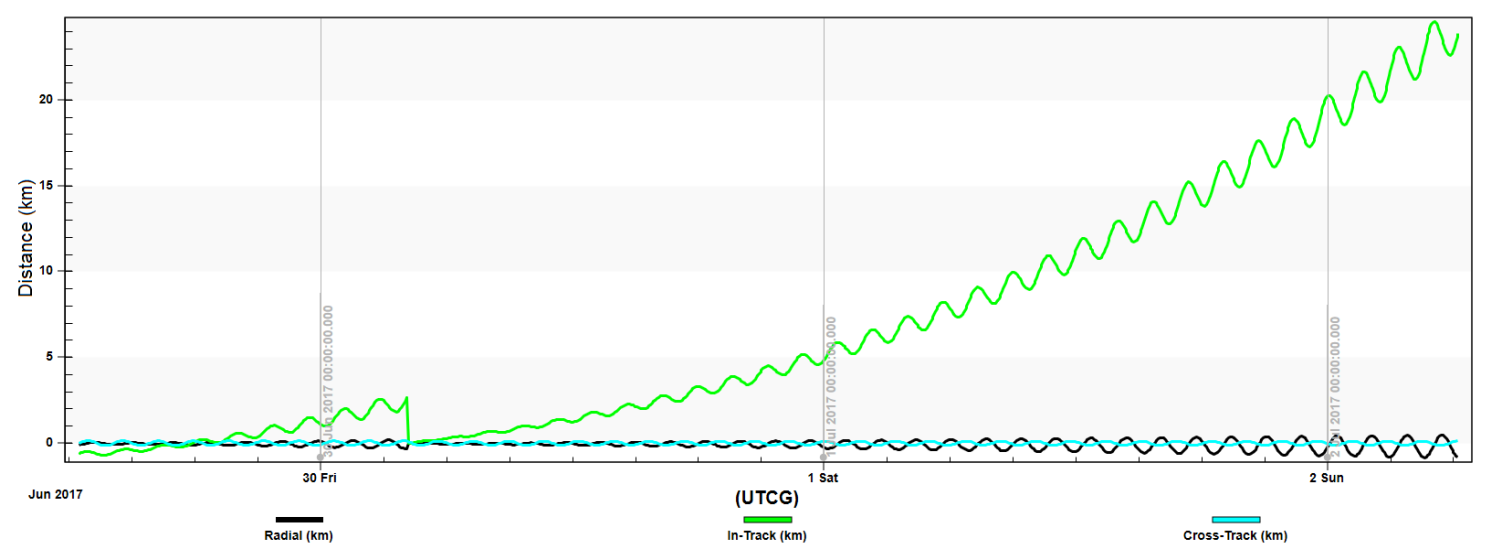

Figure 5.14: UniBRITE-1 Opportunity \#1 Method 2, 3- $\sigma$ filter RIC Comparison

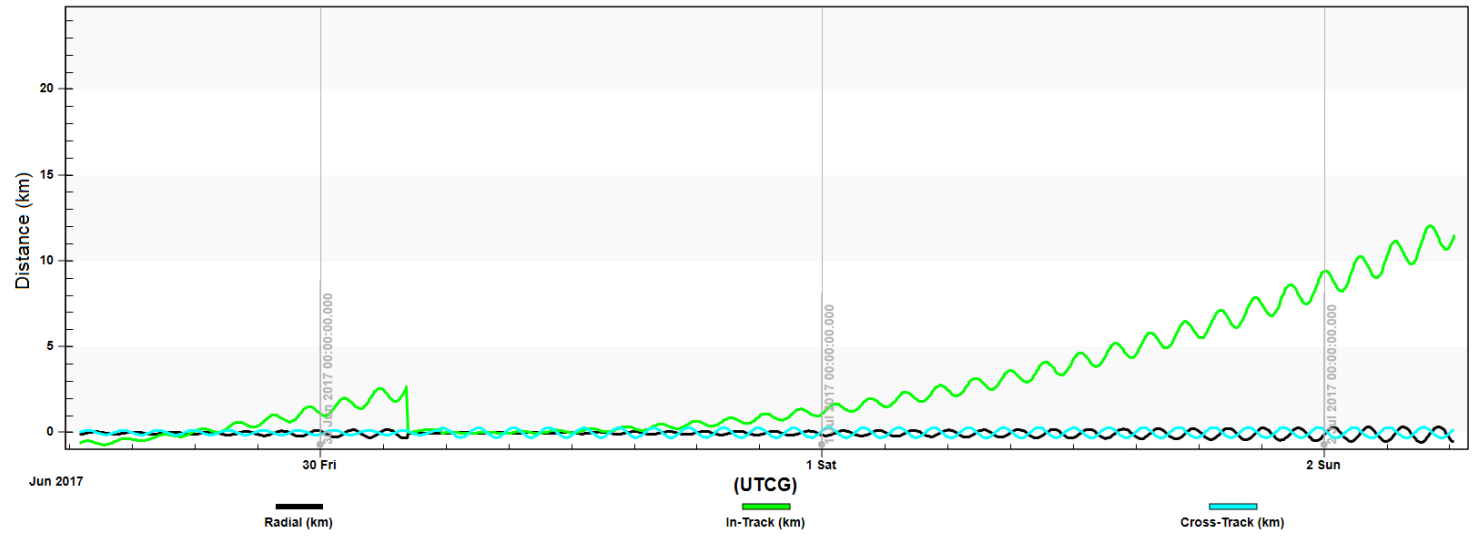

Figure 5.15: UniBRITE-1 Opportunity \#1 Method 2, 5- $\sigma$ filter RIC Comparison

These results are similar to those from BriteAustria. It appears that the quality of the TLE and it's associated uncertainty may have been too restrictive. By allowing the additional observations into the filter, a superior orbit estimate was created closer to the true orbit state. This migration is visualized by the orbit fits and their associated covariances in Fig. 5.16.

\subsubsection{LVLH}

The generated orbit ephemeris for Methods 1 and 2 were exported from STK and were compared to the correpsonding SP ephemeris in LVLH in Fig. 5.17. The results are similar to the findings from BriteAustria, although the accuracy from the orbit estimates were switched. In this case, the estimate from Method 2 orbit was a closer 


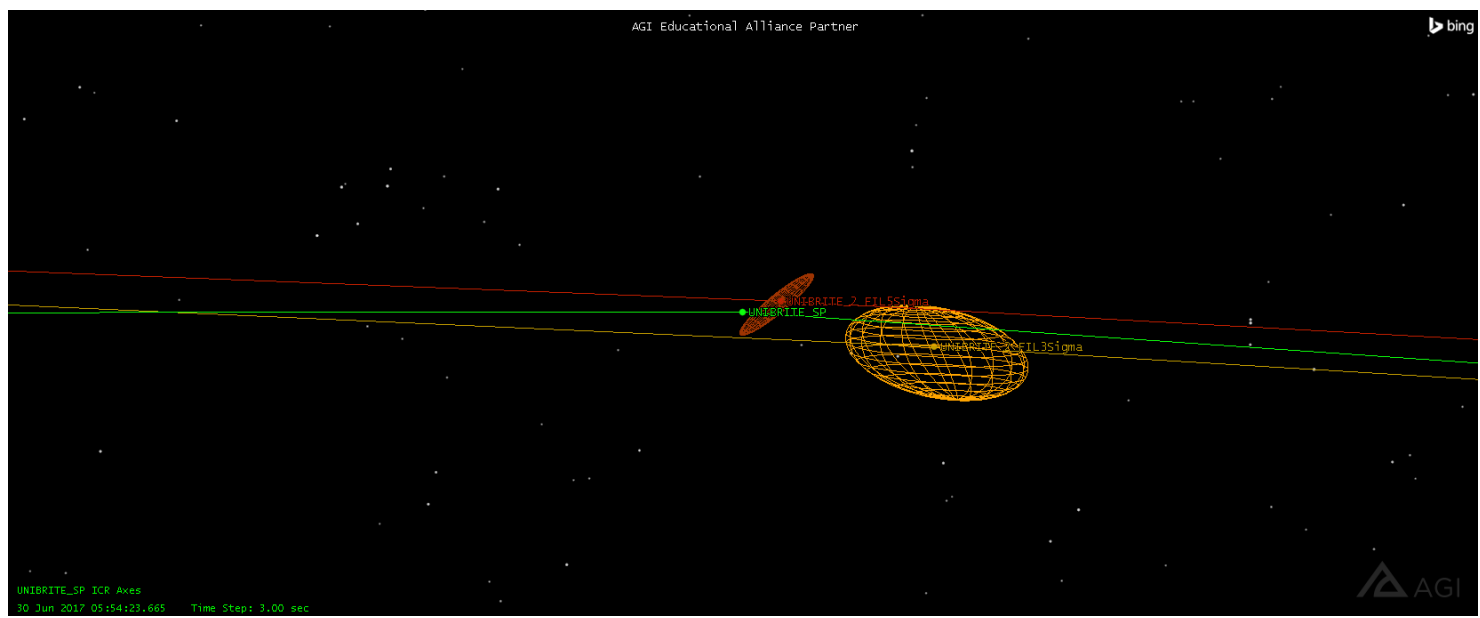

Figure 5.16: UniBRITE-1 Opportunity \#1 Method 2, Orbit Estimates

fit than that of Method 1. This result is what was expected provided the lack of confidence in the Method 1 orbit, as well as the in-track velocity mis-match. Again, this transformation preserves the relative position information and can be used to examine their relationships for exploitation. A comparison of the error in the orbit fits can be seen in Fig. 5.18. 
SP, Method 1, \& Method 2 Orbit in NEOSSat LVLH (X \& Y)

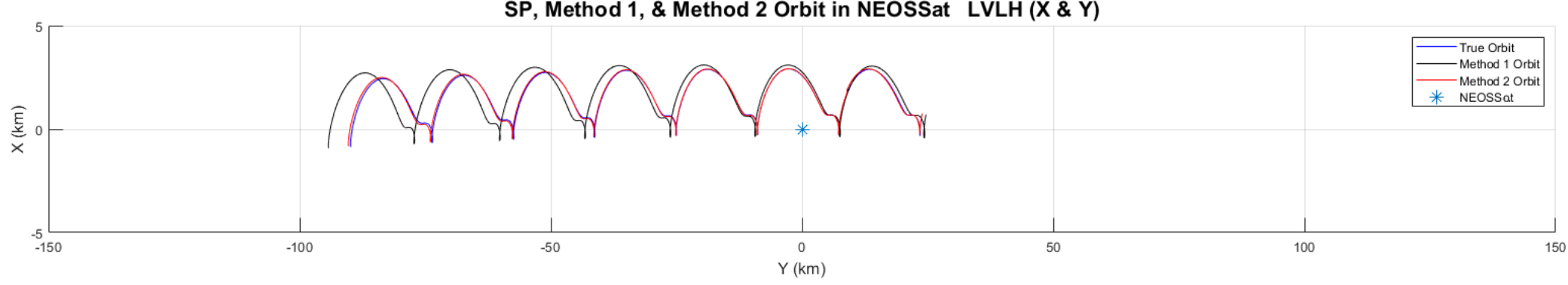

SP, Method 1, \& Method 2 Orbit in NEOSSat LVLH (X \& Z)

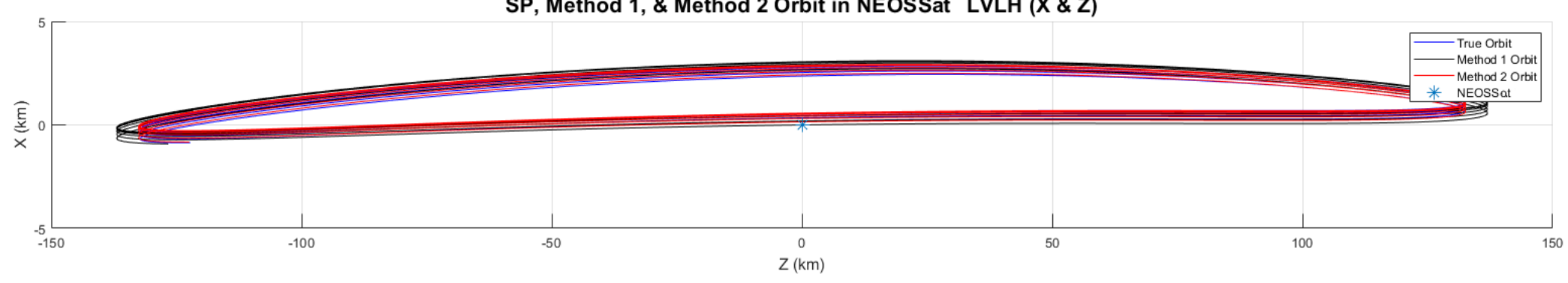

SP, Method 1, \& Method 2 Orbit in NEOSSat LVLH (Z \& Y)

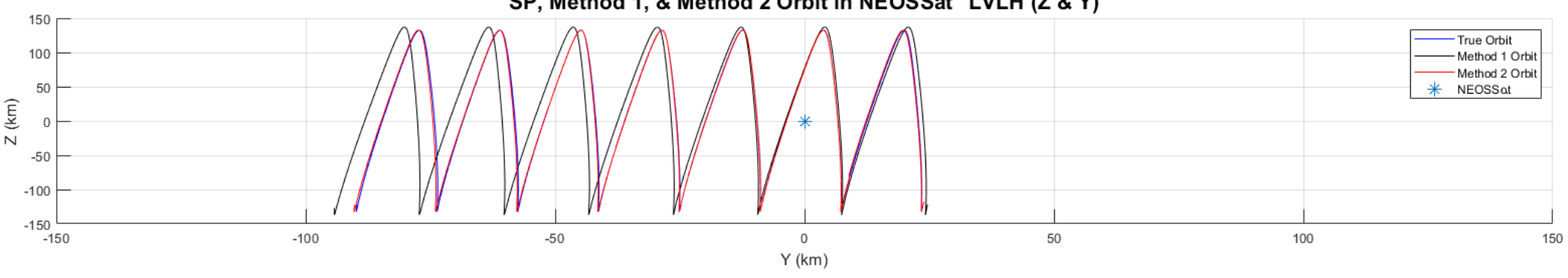

Figure 5.17: UniBRITE-1 Opportunity \#1 LVLH 


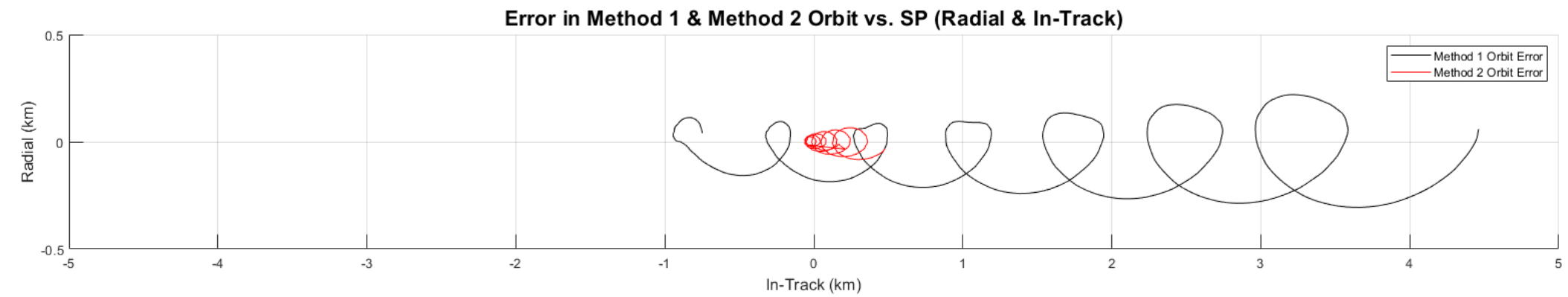

Error in Method 1 \& Method 2 Orbit vs. SP (Radial \& Cross-Track)

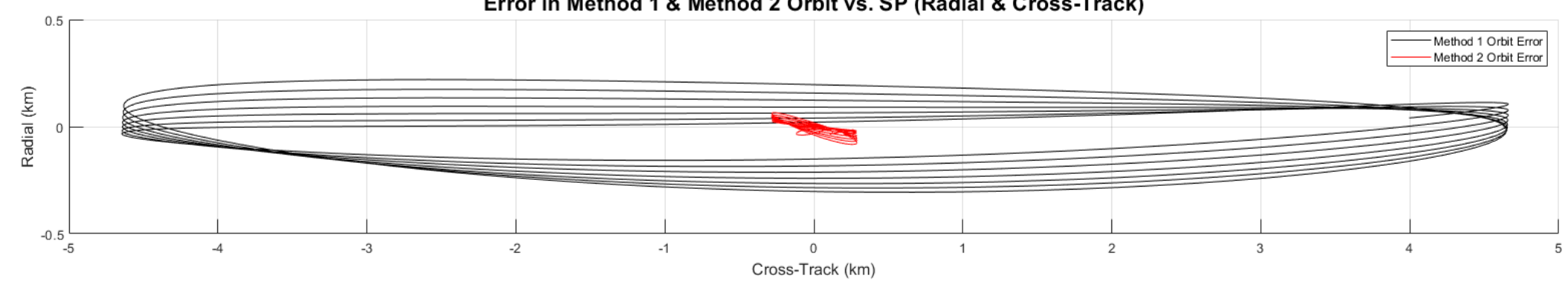

Error in Method $1 \&$ Method 2 Orbit vs. SP (Cross-Track \& In-Track)

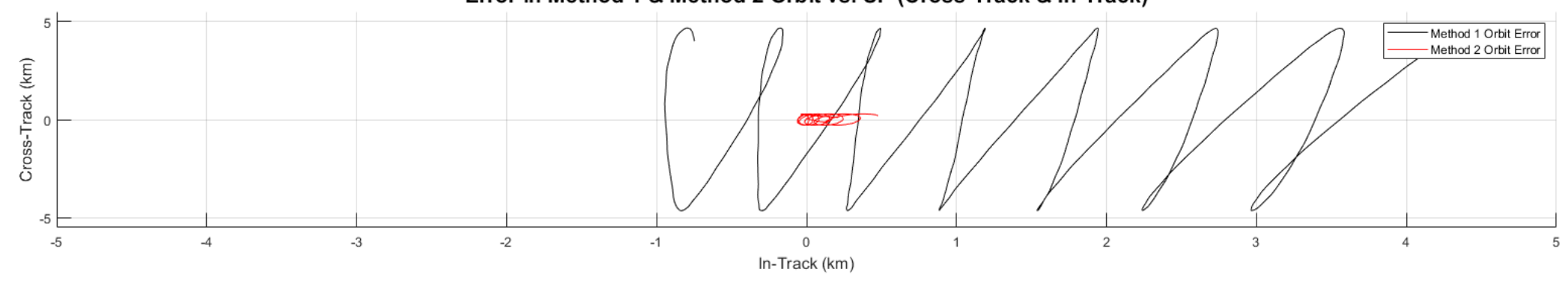

Figure 5.18: UniBRITE-1 Opportunity \#1 Orbit Estimate Errors 


\subsection{AAUSAT3 (1)}

The first proximity event with AAUSAT3 had 6 intervals which allowed sub-90 arcsec/sec angular rate tracks with NEOSSat. The first interval started at 16:13:01 UTC on 31 Aug 2017 and the final interval ended at 00:47:16 UTC on 1 Sep 2017. Within these intervals, NEOSSat was able to track and collect 28 observations, each observation containing a pair of RA and DEC measurements. During this particular event, the observations were collected on the passes as seen in Fig. 5.19 and in accordance with Table 5.3.

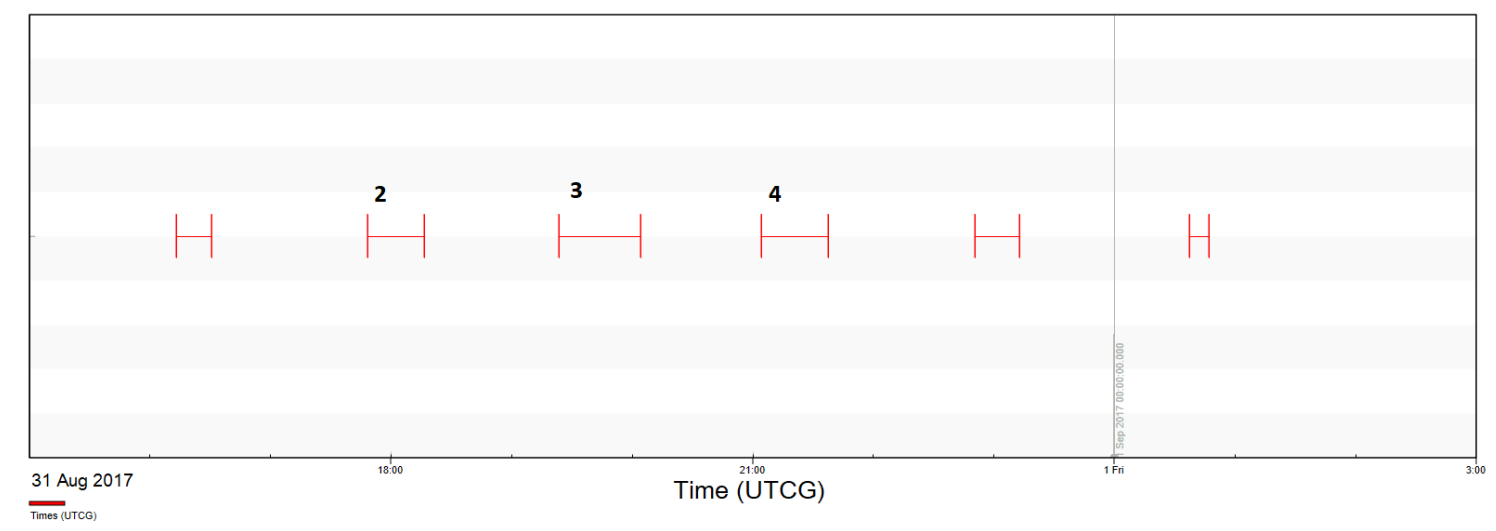

Figure 5.19: AAUSAT3 observation intervals from NEOSSat

Table 5.3: AAUSAT3 Observations

\begin{tabular}{ccccc}
\hline \hline Interval \# & Date & Start Time (UTC) & End Time (UTC) & \# of Observations \\
\hline \hline 2 & 31 Aug & $17: 53: 32$ & $18: 06: 03$ & 11 \\
3 & 31 Aug & $19: 25: 32$ & $19: 38: 05$ & 11 \\
4 & 31 Aug & $21: 17: 04$ & $21: 19: 34$ & 6 \\
\hline \hline
\end{tabular}

\subsubsection{Method 1}

The observations in all intervals were insufficiently spaced to perform IODs within the individual intervals. As a result, a multi-revolution IOD using observations from intervals \#2 and \#3 were used; this method proved more challenging due to the complexity with multiple orbit solutions, but can be accomplished if care is taken. From this IOD, there were sufficient observations from the two intervals to allow for 
a DC to converge and create a resulting orbit with a covariance matrix representing the uncertainty in the orbit estimate. By fitting the orbit in this range, it allowed for observations from interval \#4 to be ingested into the resulting filter seeded by the results of the DC process. The resulting orbit of the filter was compared to the reference SP orbit and compared in RIC in Fig. 5.20.

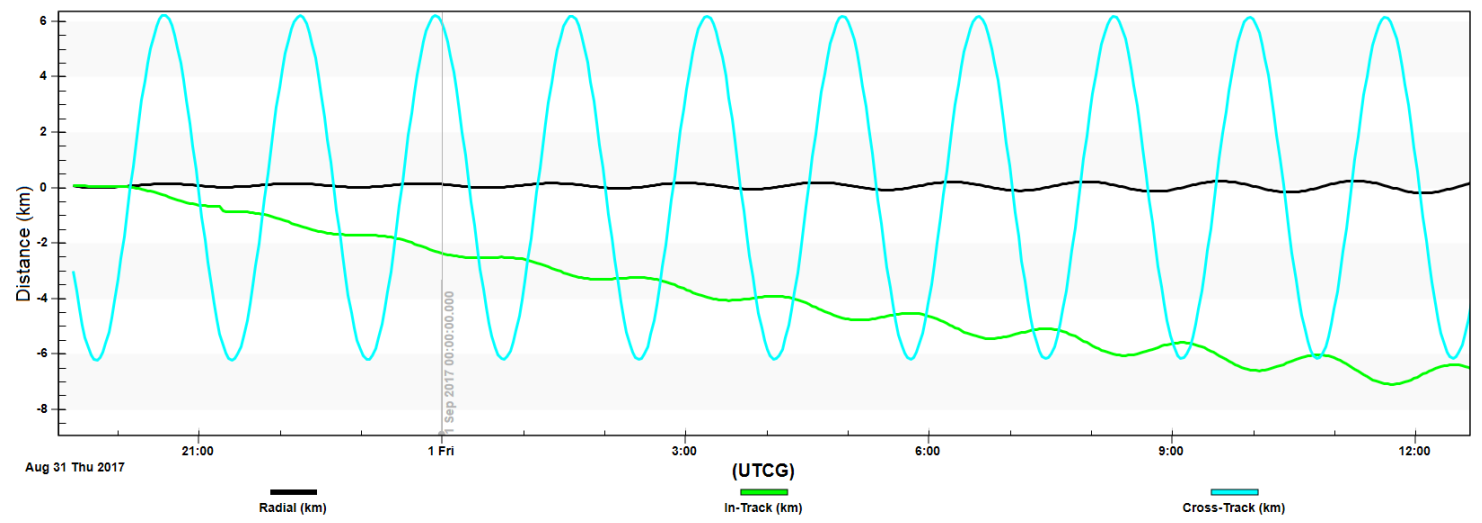

Figure 5.20: AAUSAT3 Opportunity \#1 Method 1, RIC Comparison

From the resulting orbit fit, it can be seen that the orbit has a great deal of accuracy in the radial component, yet lacks the corresponding accuracy in the crosstrack component. Over the course of the observations, the satellite varied in distance from 130-240 km with respect to NEOSSat. In addition, there is again a mis-match for the in-track velocity as that component grows over time. The discontinuity in Fig. 5.20 at approximately $21: 00$ is the filter updating the state estimate with the observations from interval \#4, all of which were accepted with the $3-\sigma$ tolerance. The $1-\sigma$ confidence from the diagonal components in RIC of the LS covariance matrix are: R:95.5 m, I:3320.3 m, C:16227 m, reinforcing the significant lack of accuracy in the in-track and cross-track components with a significant lack of overall confidence in the orbit estimate.

Similar to the results from UniBRITE-1, this orbit fit would likely prompt a review of the orbit estimate by manually rejecting observations in order to tighten the resulting covariance matrix and orbit estimate. 


\subsubsection{Method 2}

The seeded orbit state for the satellite came from a TLE with an epoch of 05:28:49 UTC on 31 Aug 2017, approximately 12 hours before the observations, and given estimated orbit uncertainties of R:102 m, I:471 m, C:126 m [16]. When running the filter with a rejection tolerance of $3-\sigma$, a significant number of the observations from interval \#3 were rejected, while intermittent observations from intervals \#2 and \#4 were accepted. When re-running the filter with the rejection tolerance of 4- $\sigma$, all observations were accepted. What is most interesting is that there was no significant change in the quality of the orbit as a result of opening the filter. The RIC comparisons to the SP ephemerides for the two runs can be seen in Figs. 5.21 and 5.22 .

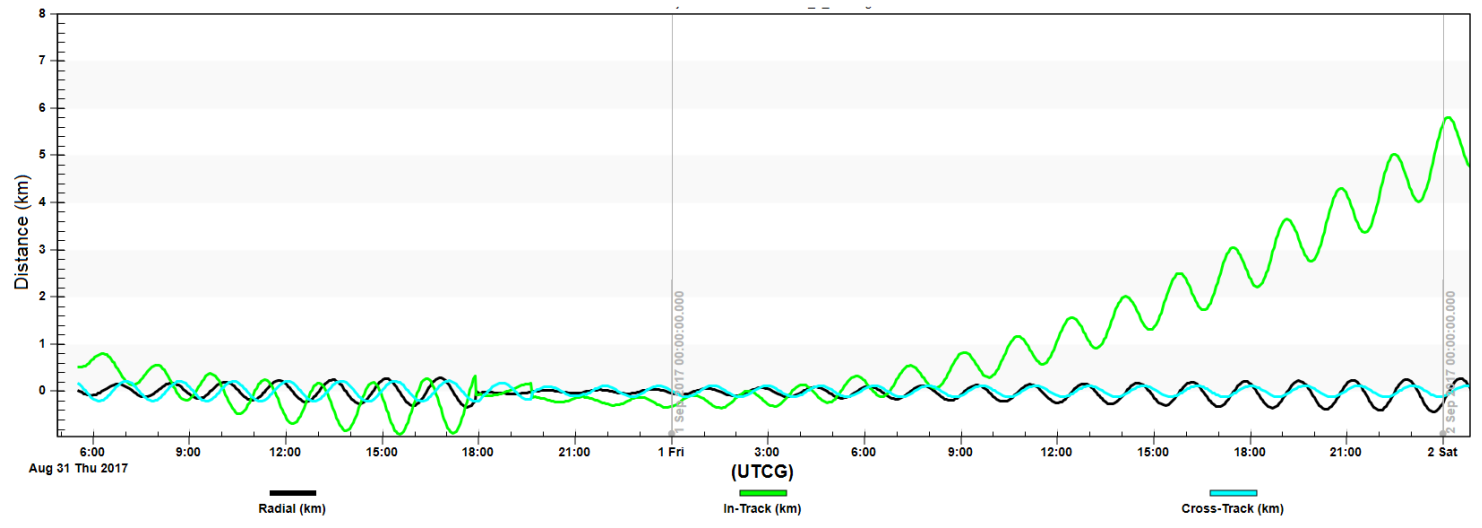

Figure 5.21: AAUSAT3 Opportunity \#1 Method 2, 3- $\sigma$ filter RIC Comparison

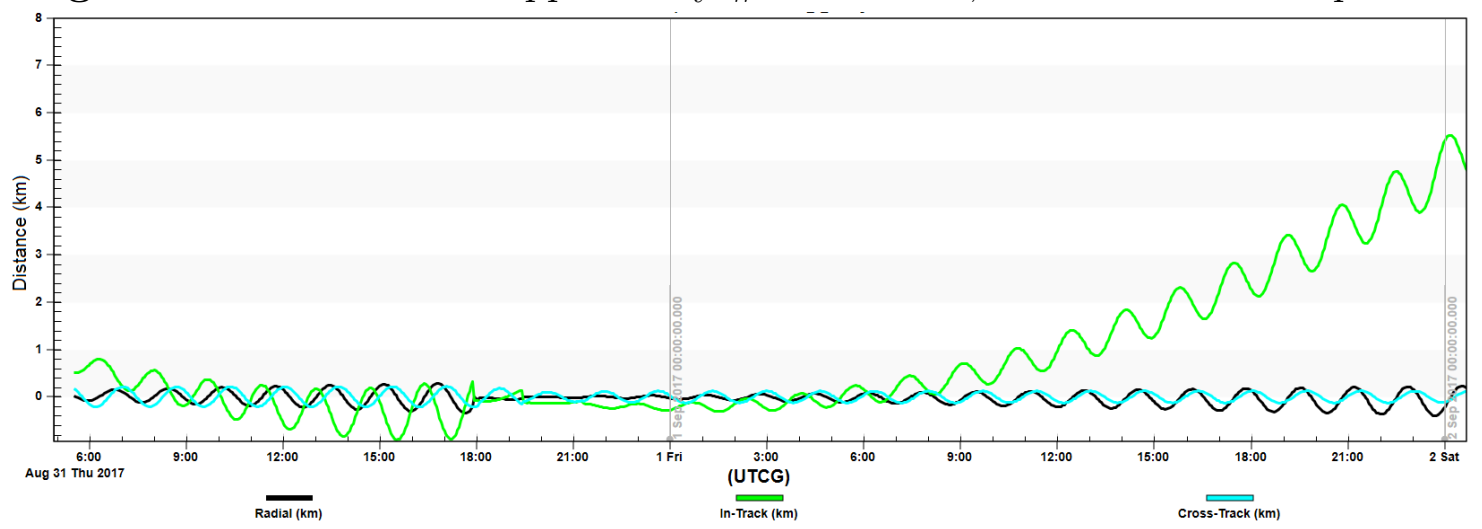

Figure 5.22: AAUSAT3 Opportunity \#1 Method 2, 4- $\sigma$ filter RIC Comparison 
With AAUSAT3, it appears as that the quality of the TLE and associated uncertainty may have been too restrictive. Despite running two filters with different rejection tolerance levels, the resulting orbit estimates were very similar, there was no significant increase in orbit quality when compared to the associated SP ephemeris. This contrasts the results from UniBRITE-1 and BriteAustria as the lower tolerance cut-off of the filter slightly reduced the resulting orbit quality.

\subsubsection{LVLH}

The generated orbit ephemeris for Methods 1 and 2 were exported from STK and were compared to the correpsonding SP ephemeris in LVLH in Fig. 5.23. The results from this method were very similar to the findings from UniBRITE-1. What is interesting in the LVLH plot is the resulting triangular pattern when looking at the third subplot of Fig. 5.23. This pattern is a result of the larger relative velocity between the satellite as AAUSAT3's semimajor axis is smaller than NEOSSat's. This difference in semimajor axes between satellite was larger than previous events, and therefore AAUSAT3 was moving more rapidly past NEOSSat in comparison to the previous satellite. Despite this difference, the LVLH transformation preserves the relative position information and can be used to examine their relationships for exploitation. A comparison of the error in the orbit fits can be seen in Fig. 5.24. 
SP, Method 1, \& Method 2 Orbit in NEOSSat LVLH (X \& Y)

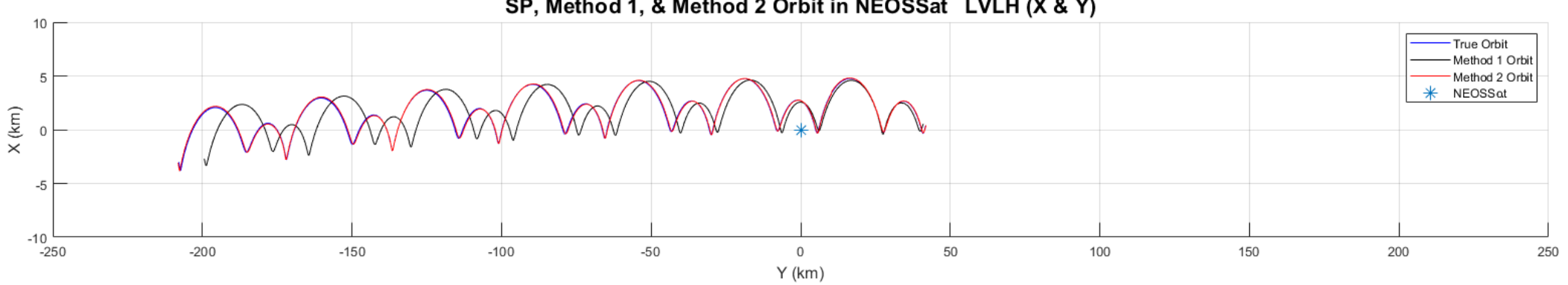

SP, Method 1, \& Method 2 Orbit in NEOSSat LVLH (X \& Z)

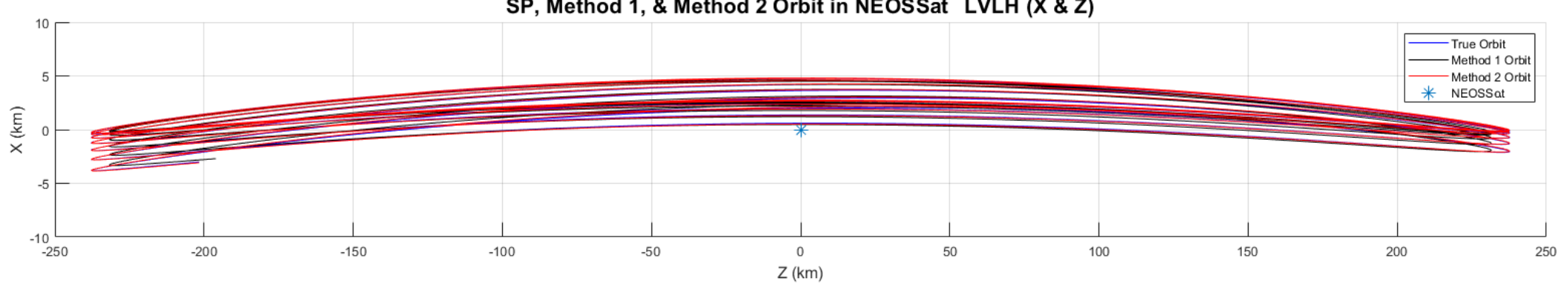

SP, Method 1, \& Method 2 Orbit in NEOSSat LVLH (Z \& Y)

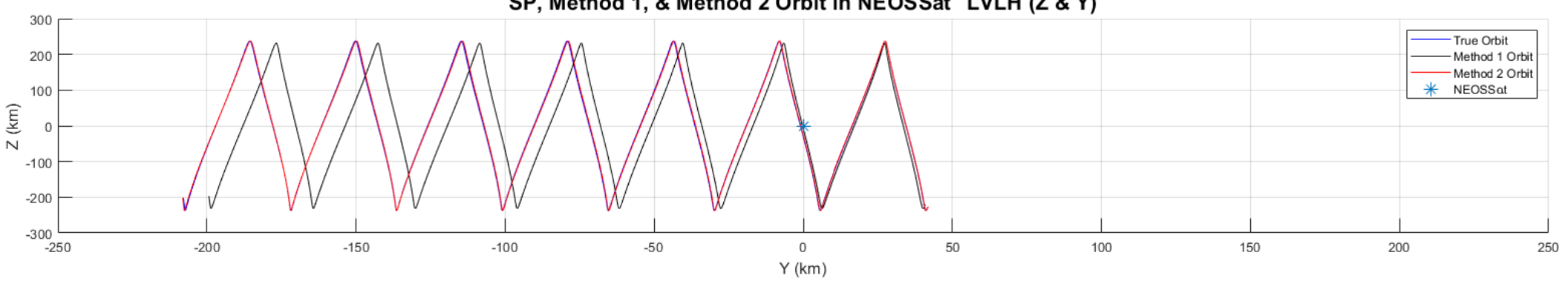

Figure 5.23: AAUSAT3 Opportunity \#1 LVLH 


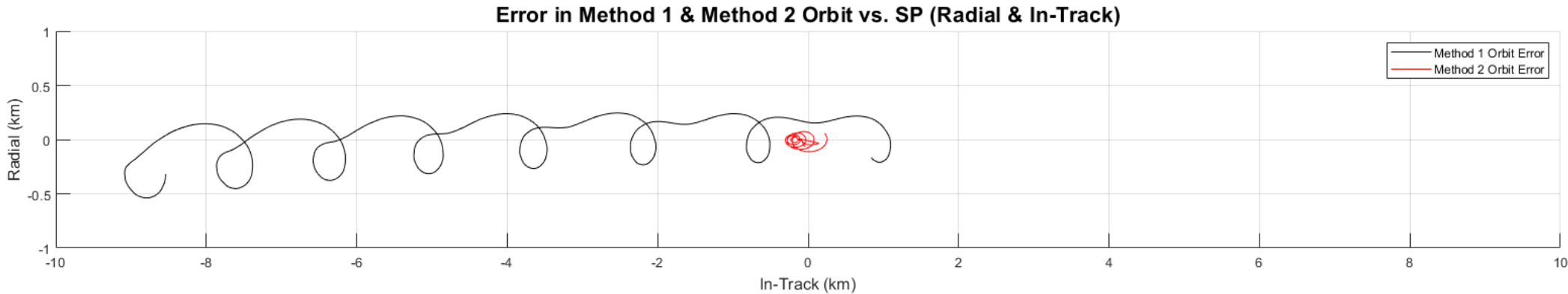

Error in Method 1 \& Method 2 Orbit vs. SP (Radial \& Cross-Track)

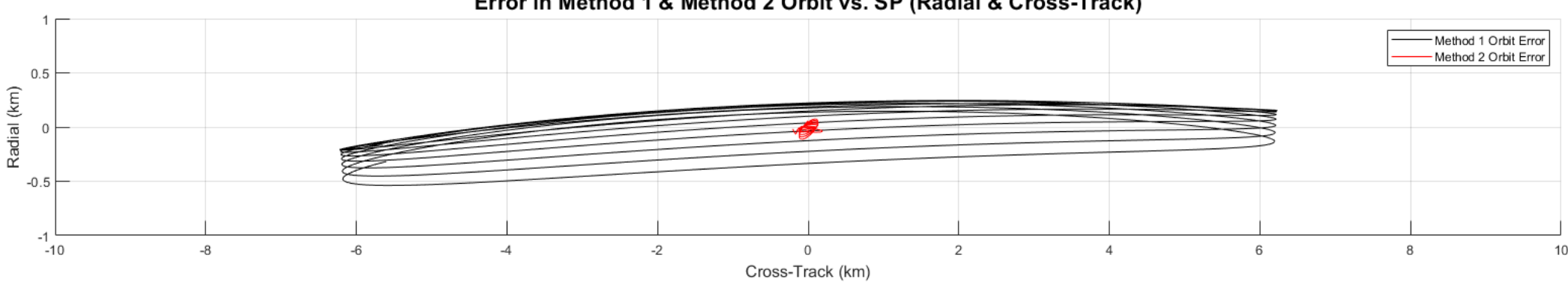

Error in Method 1 \& Method 2 Orbit vs. SP (Cross-Track \& In-Track)

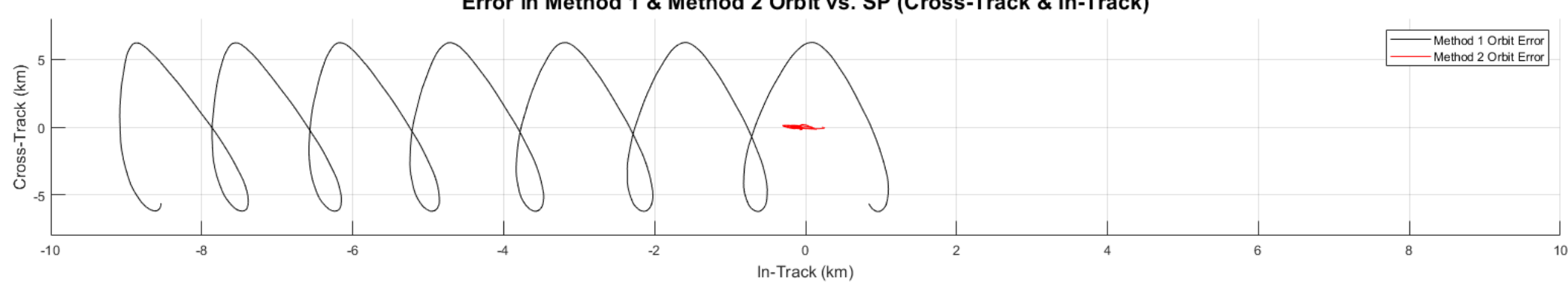

Figure 5.24: AAUSAT3 Opportunity \#1 Orbit Estimate Errors 


\subsection{SARAL}

The proximity event with SARAL had a significantly higher number of intervals which allowed sub-90 arcsec/sec angular rate tracks with NEOSSat. The first interval started at 00:56:06 UTC on 8 Sep 2017 and the final interval ended at 03:50:41 UTC on 9 Sep 17. Within these intervals, NEOSSat was able to track and collect 60 observations, each observation containing a pair of RA and DEC measurements. During this particular event, the observations were collected on the passes as seen in Fig. 5.25 and in accordance with Table 5.4.

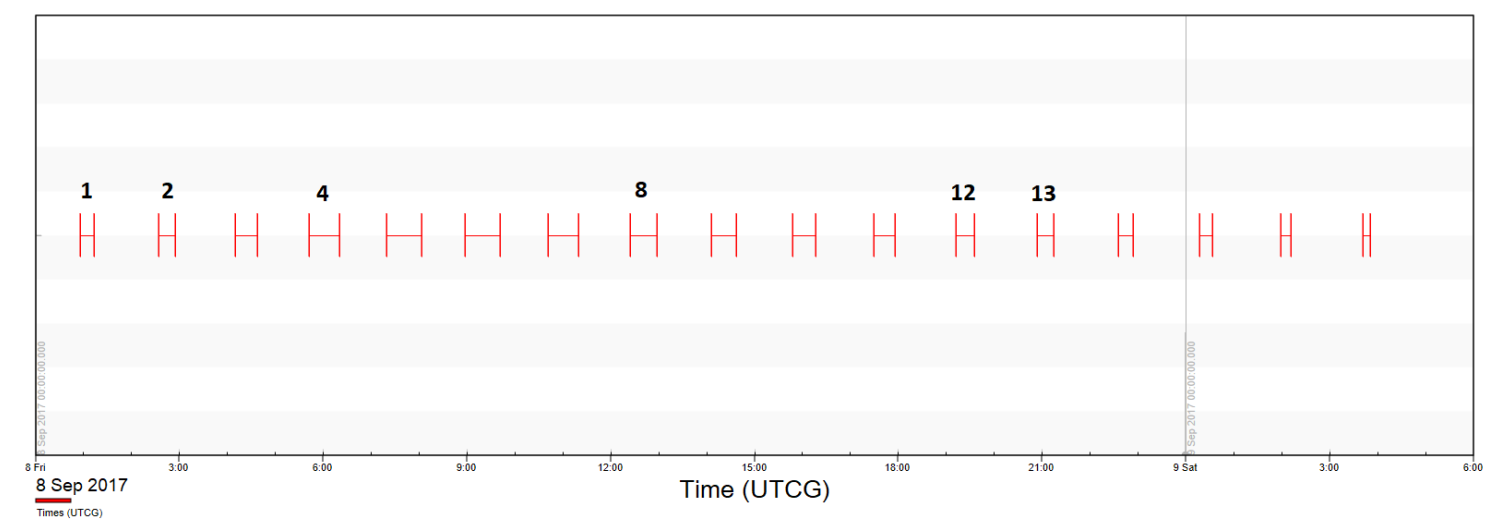

Figure 5.25: SARAL observation intervals from NEOSSat

Table 5.4: Saral Observations

\begin{tabular}{ccccc}
\hline \hline Interval \# & Date & Start Time (UTC) & End Time (UTC) & \# of Observations \\
\hline \hline 1 & 8 Sep & $00: 56: 06$ & $01: 10: 02$ & 10 \\
2 & 8 Sep & $02: 36: 04$ & $02: 48: 33$ & 12 \\
4 & 8 Sep & $05: 43: 02$ & $06: 05: 35$ & 17 \\
8 & 8 Sep & $12: 27: 02$ & $12: 29: 32$ & 6 \\
12 & 8 Sep & $19: 18: 02$ & $19: 29: 32$ & 10 \\
13 & 8 Sep & $21: 02: 02$ & $21: 04: 32$ & 5 \\
\hline \hline
\end{tabular}

\subsubsection{Method 1}

The observations within interval \#1 were sufficiently spaced to produce an IOD. From this IOD, there were also sufficient observations on interval \#1 to allow for a DC to converge and create a resulting orbit with a covariance matrix. By fitting the orbit in 
this range, it allowed for observations from the five subsequent intervals to be ingested into the resulting filter seeded from the DC process. The resulting orbit of the filter was compared to the reference ILRS orbit and compared in RIC in Fig. 5.26.

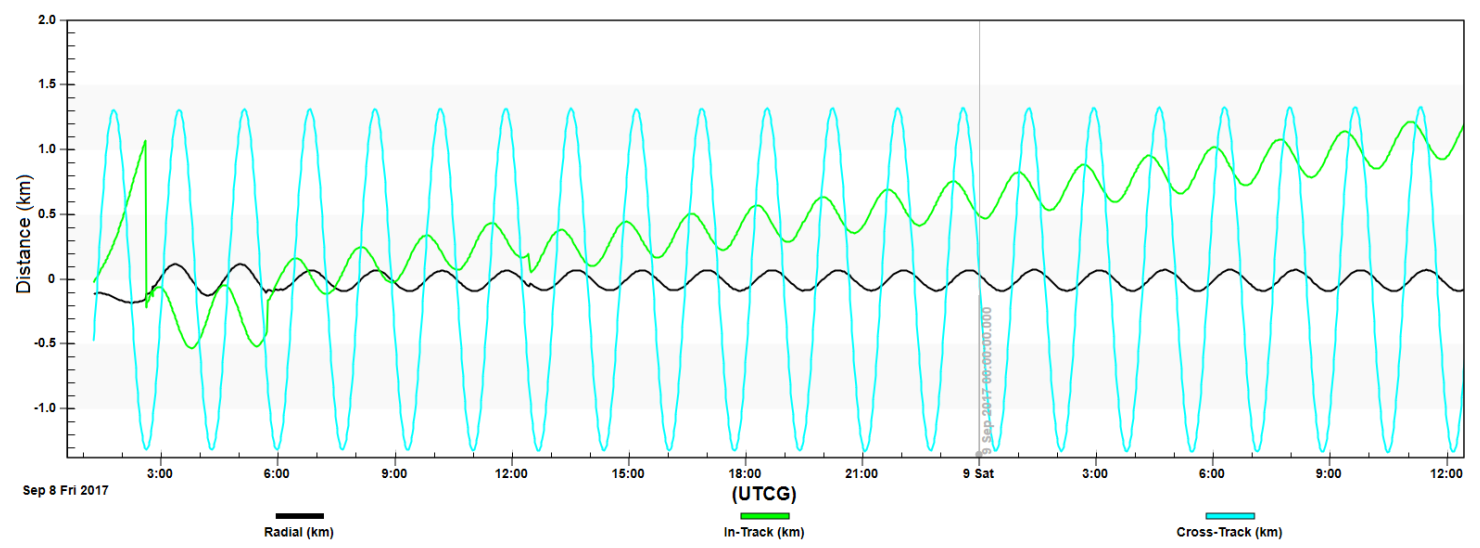

Figure 5.26: SARAL Opportunity \#1 Method 1 RIC Comparison

From the initial orbit fit, there was a significant lack of accuracy in radial, intrack and cross-track components as evident in the diagonal components of the LS covariance matrix being: R:1833.8 m, I:2172.9 m, C:11914.8 m. Over the course of the observations, the satellite varied in distance from 1062-2070 km with respect to NEOSSat. Fortunately, this fit was sufficient to allow for the filter to greatly refine the orbit estimate with the subsequent intervals, resulting in a reasonably accurate orbit fit with total separation from truth ephemerides keeping below $600 \mathrm{~m}$. Again, as seen in all previous events, there was significant uncertainty in the cross-track component and no amount of subsequent observations could refine the initial estimate, in contrast to the radial and in-track components. The reason for this inability to refine the crosstrack estimate is due to the alignment of the LOS vector with that component; the range-less measurements along this vector did not allow for direct measurements of this component.

\subsubsection{Method 2}

The seeded orbit state for the object came from a TLE with an epoch of 21:37:05 UTC on 07 Sep 2017, approximately 4 hours before the observations, and given estimated orbit uncertainties of R:102 m, I:471 m, C:126 m [16]. When running the filter with 
a rejection tolerance of $3-\sigma$, nearly half of all observations were rejected. When rerunning the filter with the rejection tolerance of $5-\sigma$, all observations were accepted. The RIC comparisons for the two runs can be seen in Figs. 5.27 and 5.28.

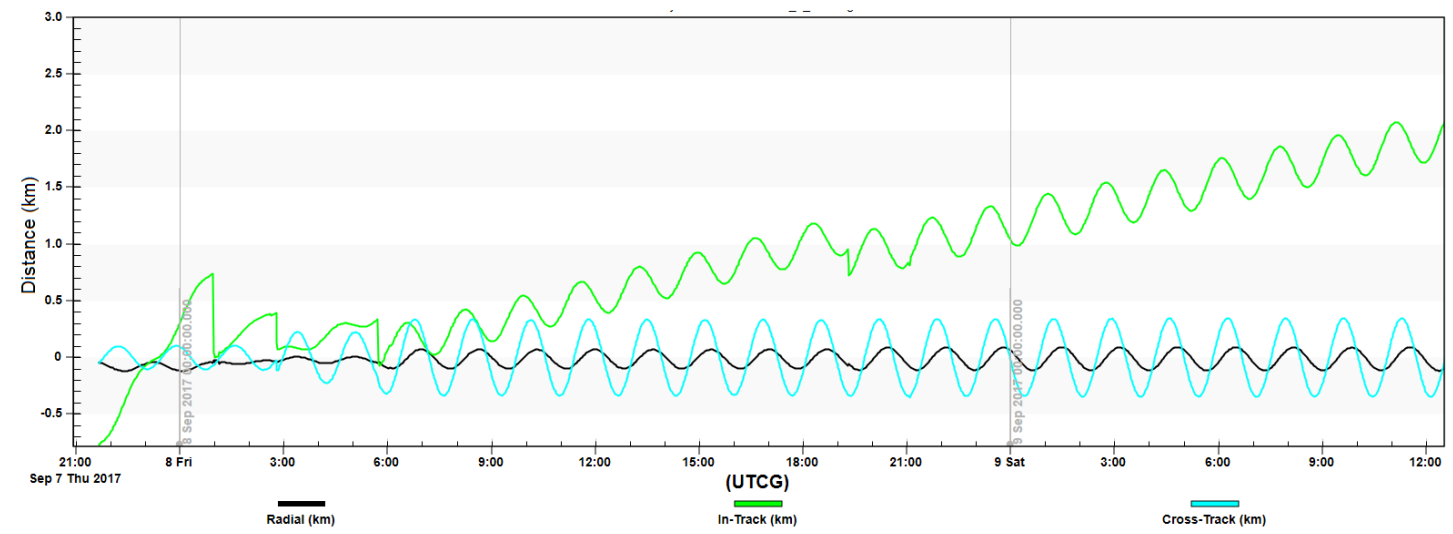

Figure 5.27: SARAL Opportunity \#1 Method 2, 3- $\sigma$ filter RIC Comparison

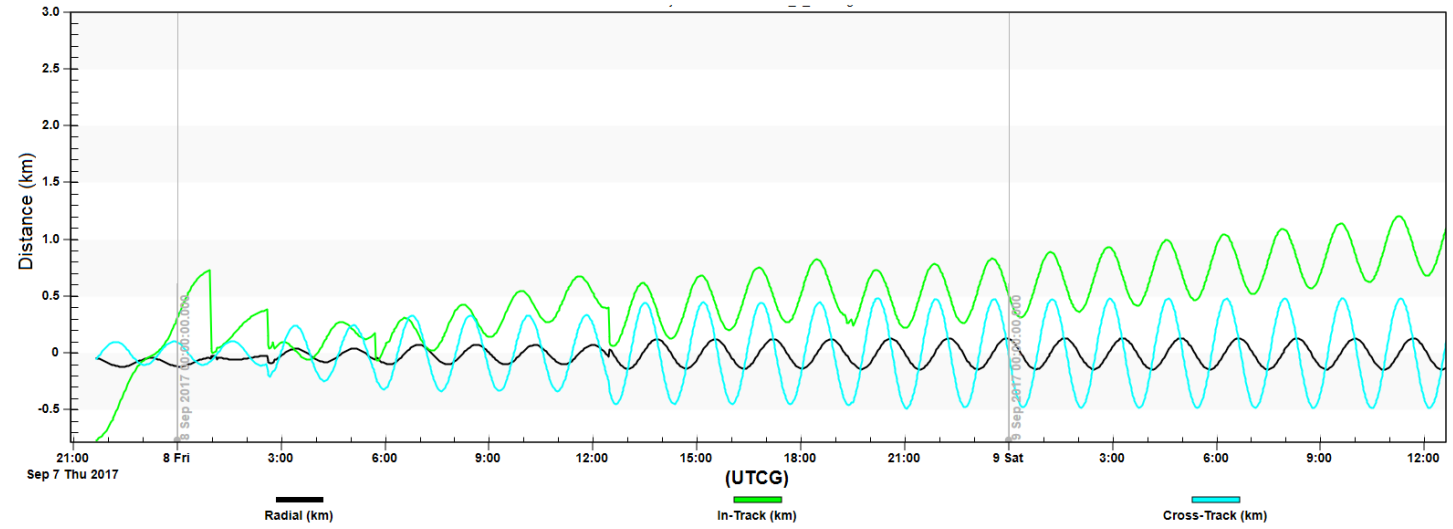

Figure 5.28: SARAL Opportunity \#1 Method 2, 5- $\sigma$ filter RIC Comparison

Once again, the similar results of the TLE and associated uncertainty may have been too constricting. Running a filter with a larger tolerance allowed for a better refinement of the position. It is important to note that in this case, the RIC error of the SARAL ephemeris can be quantified at R:1 cm I:5 cm C:1 cm; several orders of magnitude lower than the offset of positions in Figs. 5.27 and 5.28. Of interest is the increase of the cross-track component offsets as the filter is updated; these findings are in contrast to previous Method 2 filter results. 


\subsubsection{LVLH}

The generated orbit ephemeris for Methods 1 and 2 were exported from STK and were compared to the correpsonding SP ephemeris in LVLH in Fig. 5.29. Once again, the findings are consistent with what was expected from the generated orbits, however, the pattern is significantly different. Due to the larger separation between the satellite, a translating figure-eight pattern became apparent as the satellite oscillated past NEOSSat due to the slight mis-matches in orbital periods. Due to this larger separation, the scale of any position or velocity mis-match is dwarfed by the significantly greater distance between the two satellite. Again, this transformation preserves the relative position information and can be used to examine their relation-

ships for exploitation. A comparison of the error in the orbit fits can be seen in Fig. 5.30 . 


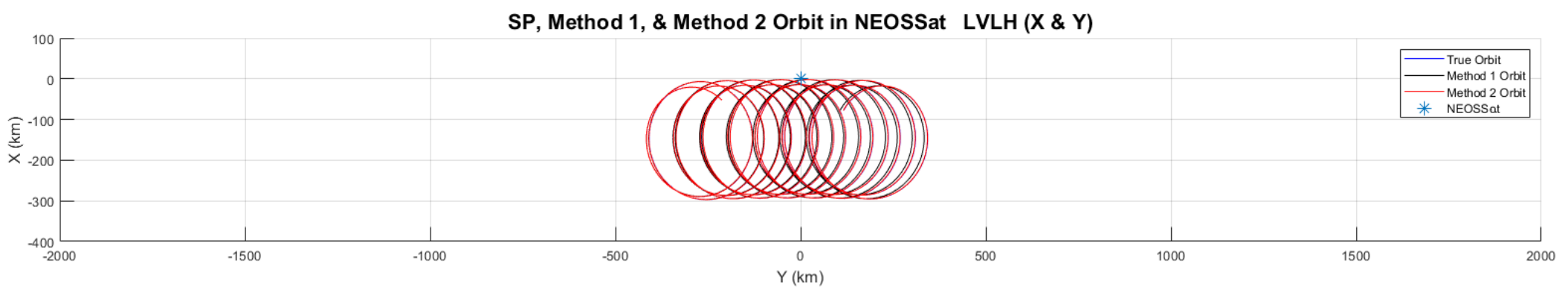

SP, Method 1, \& Method 2 Orbit in NEOSSat LVLH (X \& Z)

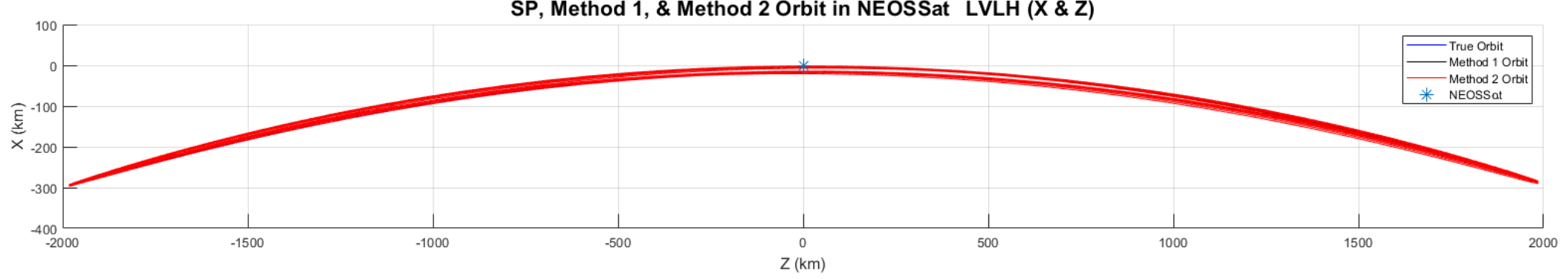

SP, Method 1, \& Method 2 Orbit in NEOSSat LVLH (Z \& Y)

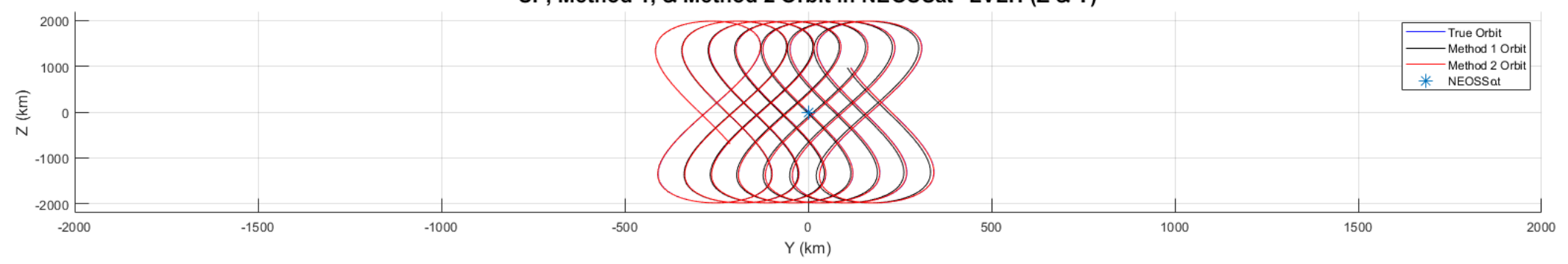

Figure 5.29: SARAL Opportunity \#1 LVLH 

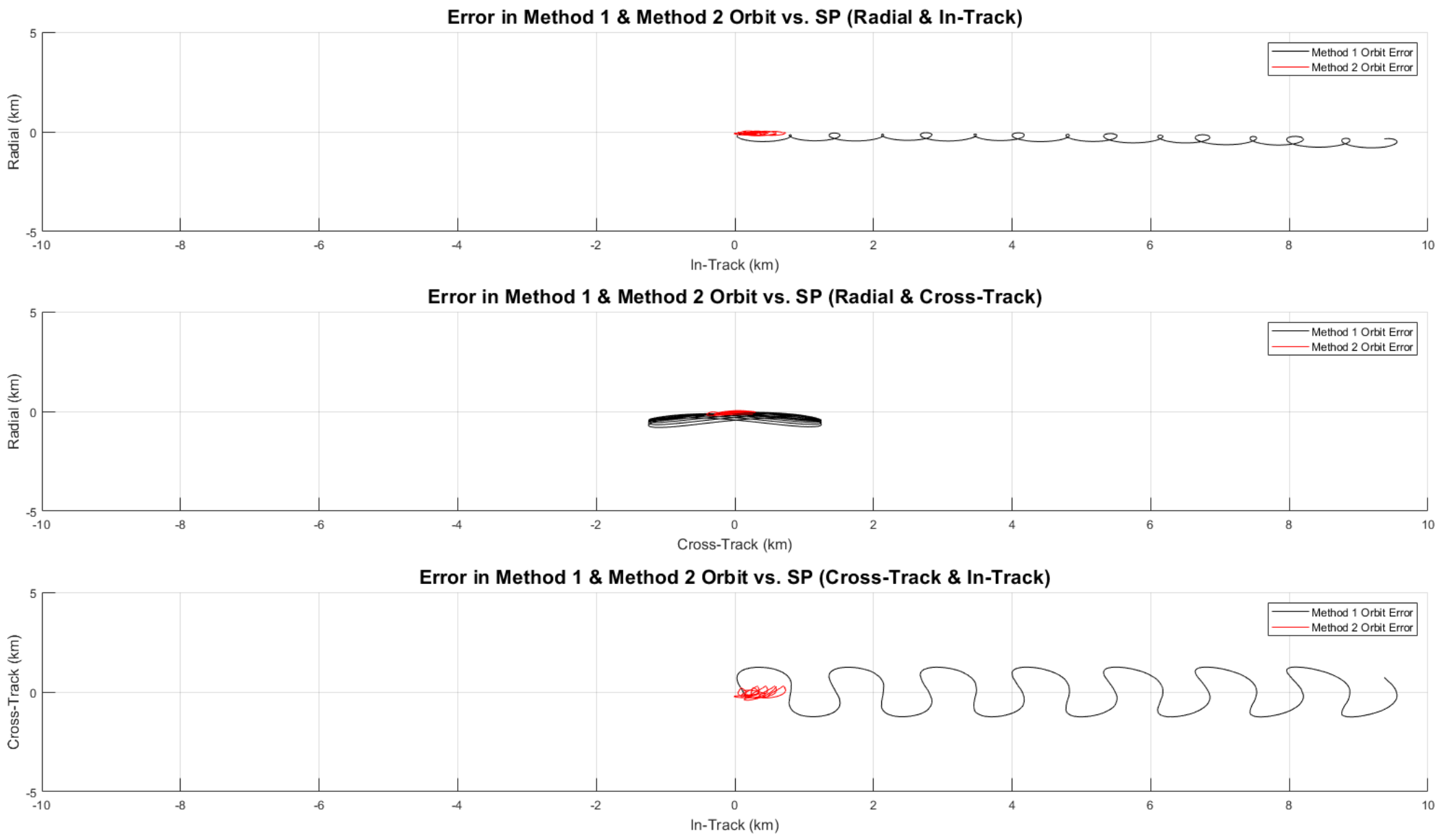

Figure 5.30: SARAL Opportunity \#1 Orbit Estimate Errors

$\infty$ 


\subsection{AAUSAT3 (2)}

The second proximity event with AAUSAT3 had 6 intervals which allowed sub-90 arcsec/sec angular rate tracks with NEOSSat. The first interval started at 03:34:16 UTC on 27 Nov 2017 and the final interval ended at 12:09:29 UTC on the same day. Within these intervals, NEOSSat was able to track and collect 6 observations, each observation containing a pair of RA and DEC measurements. During this particular event, all 6 observations were collected in the $4^{\text {th }}$ interval from 08:40:04 UTC until 08:43:49 UTC as seen in Fig. 5.31.

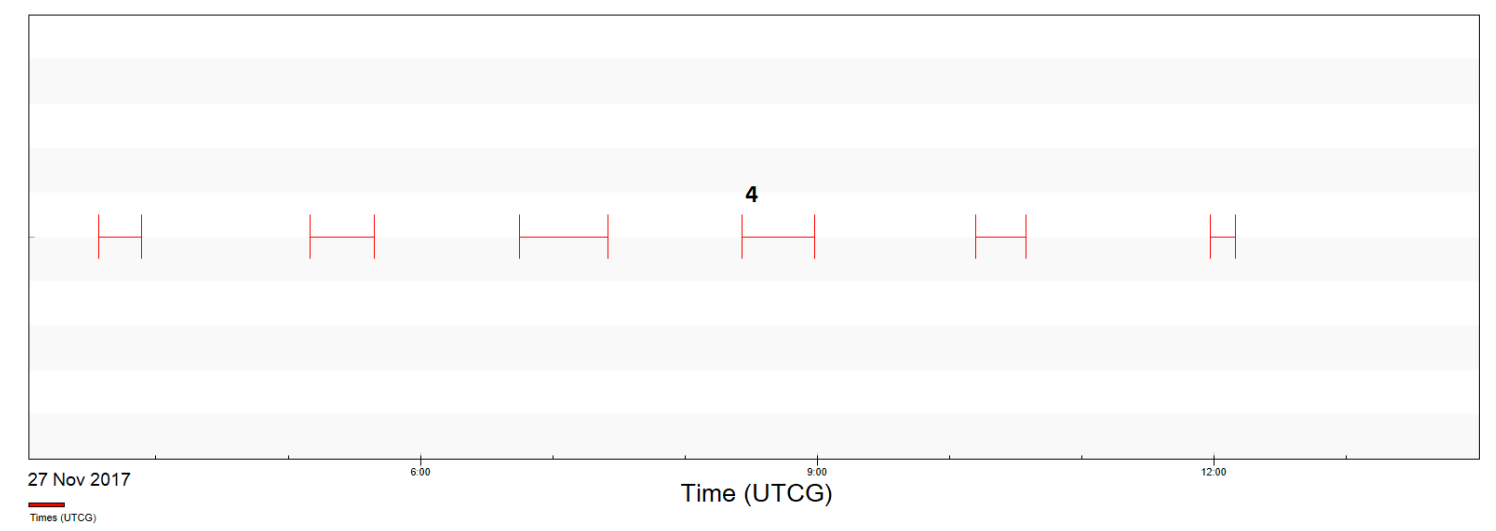

Figure 5.31: AAUSAT3 observation intervals from NEOSSat

\subsubsection{Method 1}

Unfortunately the spacing of the observations in interval \#4 was insufficient to perform an IOD. As a result, it was impossible to perform Method 1.

\subsubsection{Method 2}

The seeded orbit state for the object came from a TLE with an epoch of 04:33:01 UTC on 27 Nov 2017, approximately 4 hours before the observations, and given estimated orbit uncertainties of R:102 m, I:471 m, C:126 m [16]. Over the course of the observations, the satellite varied in distance from $240-254 \mathrm{~km}$ with respect to NEOSSat. When running the filter with a rejection tolerance of $3-\sigma$, all observations were accepted. The RIC comparisons for the run can be seen in Fig. 5.32. 


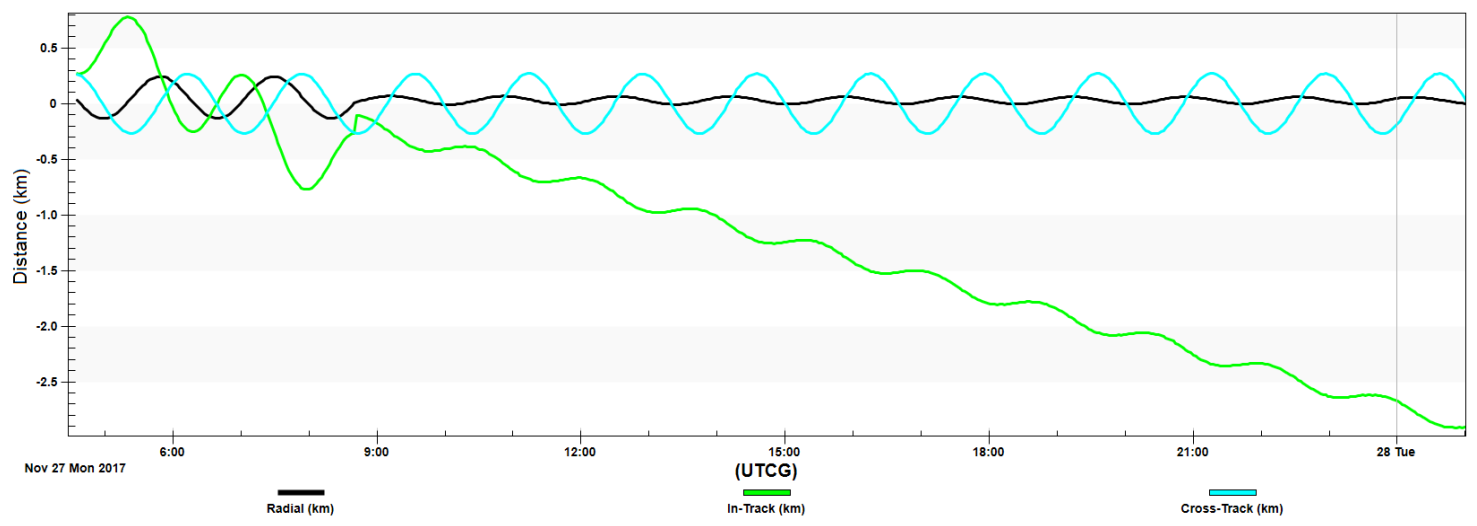

Figure 5.32: AAUSAT3 Opportunity \#2 Method 2, 3- $\sigma$ filter RIC Comparison

With the second AAUSAT3 opportunity, it appears that the quality of the TLE and associated uncertainty was sufficient to allow the observations in with a $3-\sigma$ tolerance. With the accepted observations, there was an increase in the confidence of the radial and in-track components, although no improvement in the cross-track component. The lack of update of the cross-track component is consistent with the majority of previous results.

\subsubsection{LVLH}

Due to the sparseness of data associated with this event, no LVLH examination was possible. 


\subsection{BriteAustria (2)}

The second proximity event with BriteAustria had 8 intervals which allowed sub-90 arcsec/sec angular rate tracks with NEOSSat. The first interval started at 19:48:00 UTC on 1 Dec 2017 and the final interval ended at 07:42:06 UTC on 2 Dec 2017. Within these intervals, NEOSSat was able to track and collect 12 observations, each observation containing a pair of RA and DEC measurements. During this particular event, the observations were collected on the passes as seen in Fig. 5.33 and in accordance with Table 5.5.

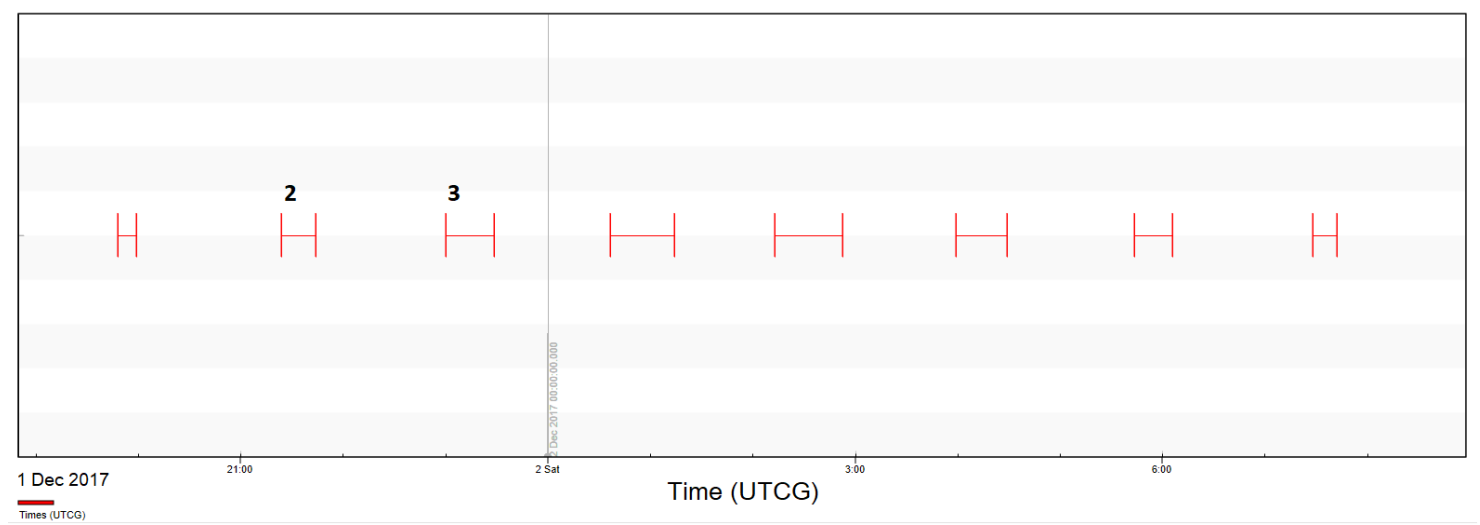

Figure 5.33: BriteAustria observation intervals from NEOSSat

Table 5.5: BriteAustria Observations

\begin{tabular}{ccccc}
\hline \hline Interval \# & Date & Start Time (UTC) & End Time (UTC) & \# of Observations \\
\hline \hline 2 & 1 Dec & $21: 29: 31$ & $21: 33: 17$ & 6 \\
3 & 1 Dec & $23: 10: 02$ & $23: 13: 48$ & 6 \\
\hline \hline
\end{tabular}

\subsubsection{Method 1}

Unfortunately the spacing of the observations in both intervals \#2 and \#3 were insufficient to perform an IOD, as well as any configuration to perform a multirevolution IOD. As a result, it was impossible to perform Method 1. 


\subsubsection{Method 2}

The seeded orbit state for the object came from a TLE with an epoch of 14:01:25 UTC on 1 Dec 2017, approximately 9 hours before the observations, and given estimated orbit uncertainties of R:102 m, I:471 m, C:126 m [16]. Over the course of the observations, the satellite varied in distance from 179-187 km with respect to NEOSSat. When running the filter with a rejection tolerance of $3-\sigma$, all observations were accepted. The RIC comparisons for the run can be seen in Fig. 5.34.

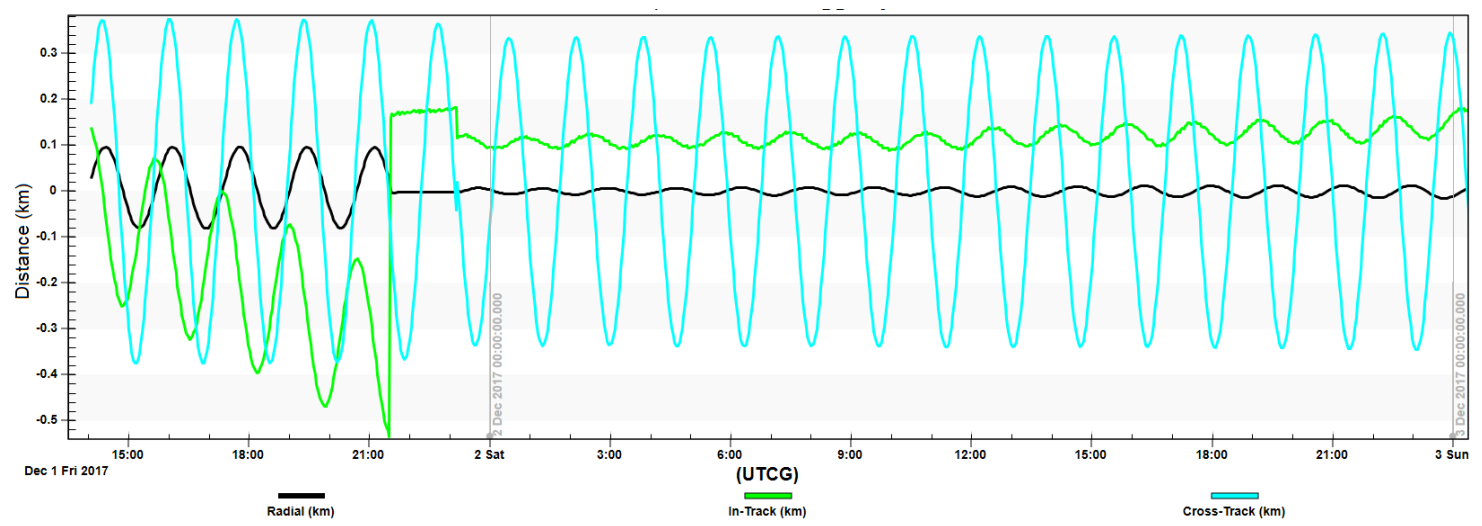

Figure 5.34: BriteAustria Opportunity \#2 Method 2, 3- $\sigma$ filter RIC Comparison

With the second BriteAustria opportunity, it appears as though the quality of the TLE and associated uncertainty was also sufficient to accept observations into the filter. This process was the most successful of the events as the orbit estimate was able to match the in-track velocity, resulting in no growth of that component; in contrast to the other tracks examined in this work. With the accepted observations, there was a significant increase in the confidence of the radial and in-track components, reducing the esimates from $100 \mathrm{~m}$ and $500 \mathrm{~m}$ to $20 \mathrm{~m}$ and $100 \mathrm{~m}$ respectively. There was no significant improvement in the cross-track component.

\subsubsection{LVLH}

Due to the sparseness of data associated with this event, no LVLH examination was undertaken. 


\subsection{QuikScat}

The proximity event with QuikScat also had 8 intervals which allowed sub-90 arcsec/sec angular rate tracks with NEOSSat. The first interval started at 10:19:37 UTC on 1 Jun 2018 and the final interval ended at 22:16:06 on the same day. Within these intervals, NEOSSat was able to track and collect 36 observations, each observation containing a pair of RA and DEC measurements. During this particular event, the observations were collected on the passes as seen in Fig. 5.35 and in accordance with Table 5.6.

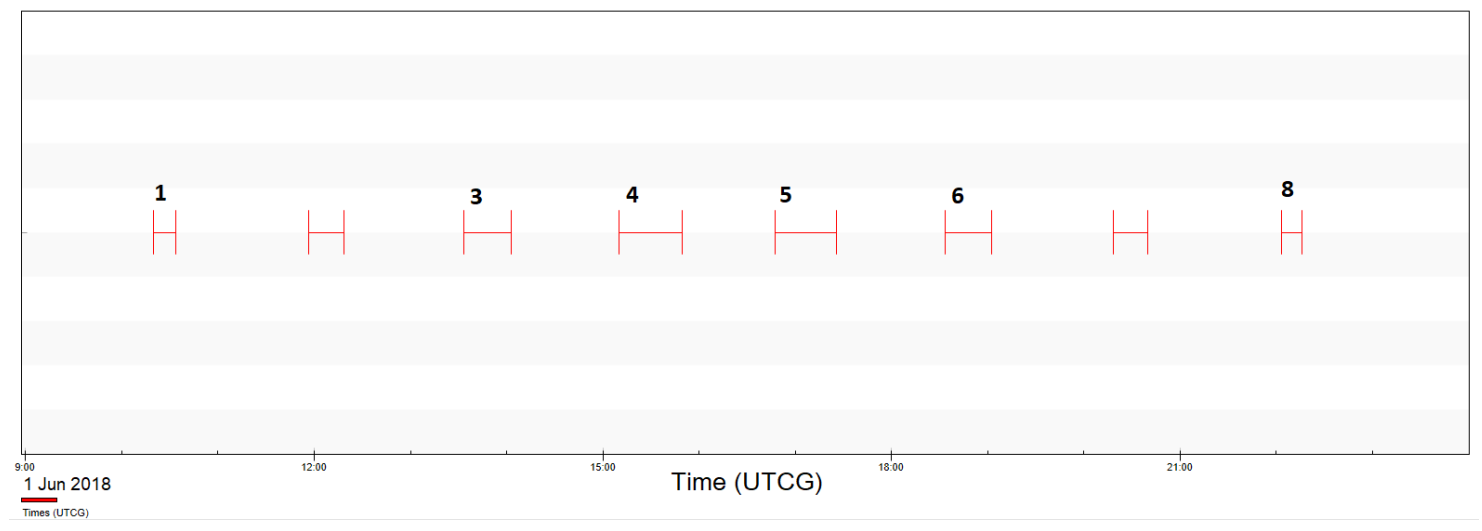

Figure 5.35: QuikScat observation intervals from NEOSSat

Table 5.6: QuikScat Observations

\begin{tabular}{ccccc}
\hline \hline Interval \# & Date & Start Time (UTC) & End Time (UTC) & \# of Observations \\
\hline \hline 1 & 1 Jun & $10: 30: 31$ & $10: 32: 31$ & 5 \\
3 & 1 Jun & $13: 43: 33$ & $13: 45: 33$ & 5 \\
4 & 1 Jun & $15: 25: 04$ & $15: 27: 04$ & 4 \\
5 & 1 Jun & $17: 14: 03$ & $17: 16: 03$ & 5 \\
6 & 1 Jun & $18: 34: 01$ & $18: 51: 03$ & 15 \\
8 & 1 Jun & $22: 04: 01$ & $22: 12: 31$ & 9 \\
\hline \hline
\end{tabular}

\subsubsection{Method 1}

Performing an IOD on the QuikScat observations were particularly challenging due to only one track of usable observations being returned per interval. As a result, the first opportunity to perform an IOD was a multi-revolution IOD over intervals \#5 
and \#6. All previous observations were then included to allow for a DC to converge and create a resulting orbit with a covariance matrix representing the uncertainty in the orbit estimate. By fitting the orbit in this way, it allowed for observations from interval \#8 to be ingested into the resulting filter seeded by the results of the DC process. The resulting orbit of the filter was compared to the reference SP orbit and compared in RIC in Fig. 5.36.

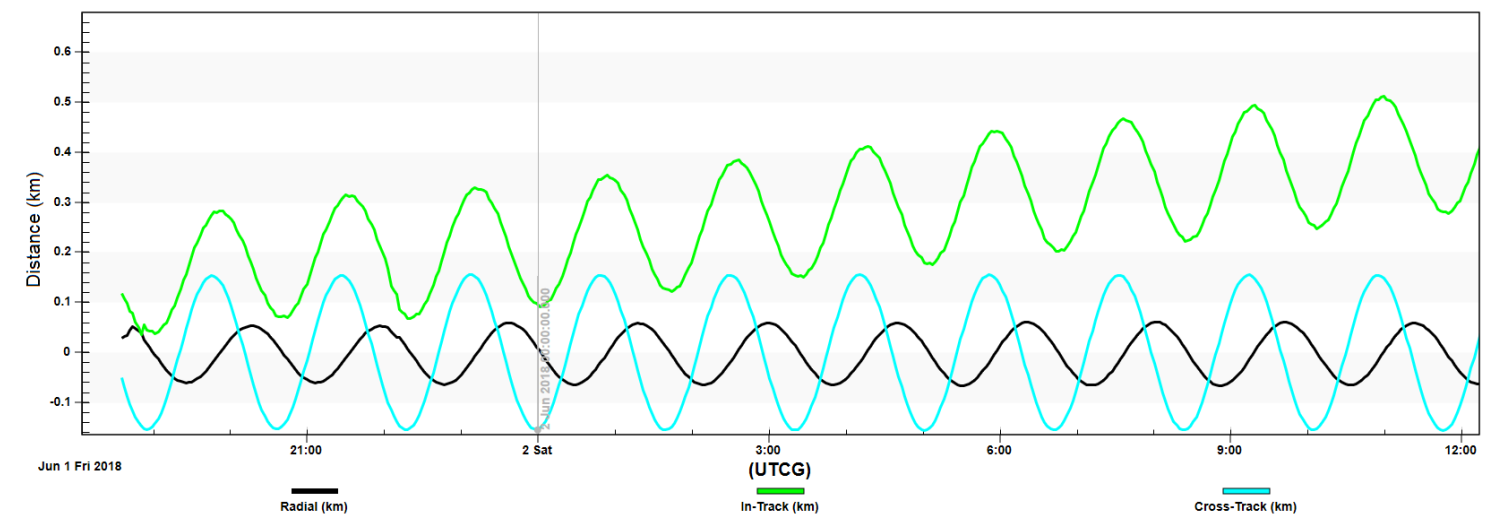

Figure 5.36: QuikScat Opportunity \#1 Method 1 RIC Comparison

From the initial orbit fit, it can be seen that there was significant accuracy in all components and that subsequent observations only slightly adjusted the orbit estimate. Over the course of observation, the satellite varied in distance from 1250$2050 \mathrm{~km}$ with respect to NEOSSat. The 1- $\sigma$ confidence from the diagonal components in RIC of the LS covariance matrix were: R:71.8 m, I:169.4 m, C:501.3 m, describing good overall confidence in the orbit fit.

\subsubsection{Method 2}

The seeded orbit state for the object came from a TLE with an epoch of 22:38:31 on 31 May 2018, approximately 12 hours before the observations, and given orbit uncertainties of R:102 m, I:471 m, C:126 m [16]. When running the filter with a rejection tolerance of $3-\sigma$, all observations were accepted. The RIC comparison for the run can be seen in Fig. 5.37.

Similar to some previous results, it appears the quality of the TLE and associated 


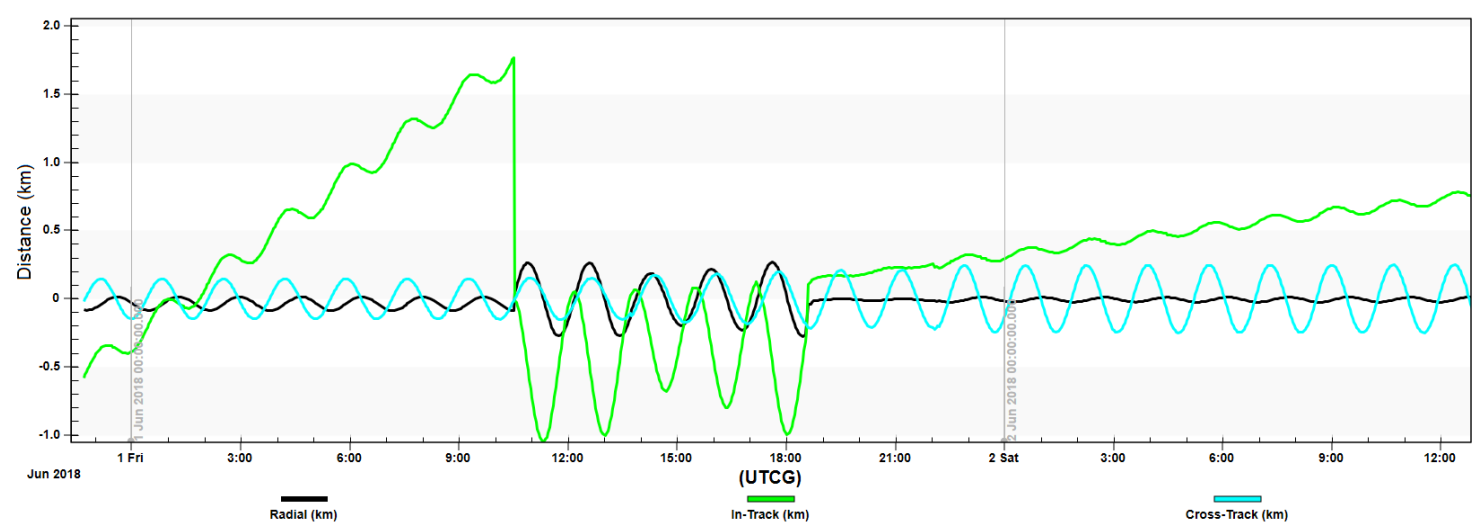

Figure 5.37: QuikScat Opportunity \#1 Method 2, 3- $\sigma$ filter RIC Comparison

uncertainty were not overly restrictive. This fact, including the frequent re-visits, allowed for a significantly more accurate orbit estimate to be generated. The results for QuikScat was similar to that of SARAL, which shares an orbit plane that is different from NEOSSat and was a significant distance away at the times of observation.

\subsubsection{LVLH}

The estimated orbit ephemeris for Methods 1 and 2 were exported from STK and were compared to the correpsonding SP ephemeris in LVLH in Fig. 5.38. The results from this method were very similar to the findings from SARAL, with the same characteristic figure-eight pattern along with any estimated position or velocity mismatchs being dwarfed by the significantly greater distance between the two satellite. Again, this transformation preserves the relative position information and can be used to examine their relationships for exploitation. A comparison of the error in the orbit fits can be seen in Fig. 5.39. 


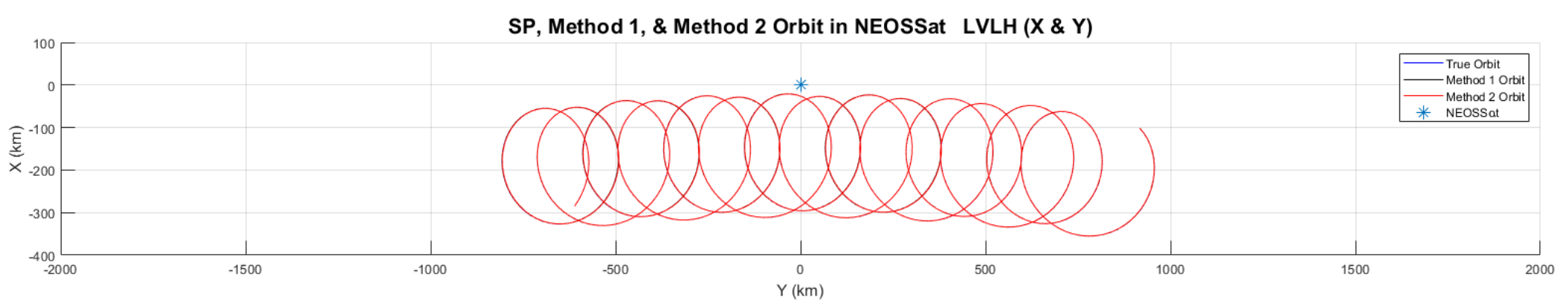

SP, Method 1, \& Method 2 Orbit in NEOSSat LVLH (X \& Z)

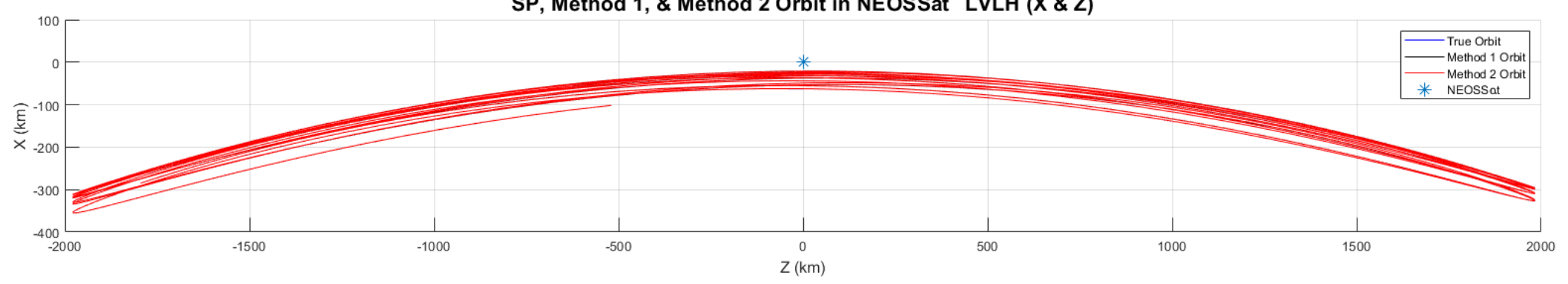

SP, Method 1, \& Method 2 Orbit in NEOSSat LVLH (Z \& Y)

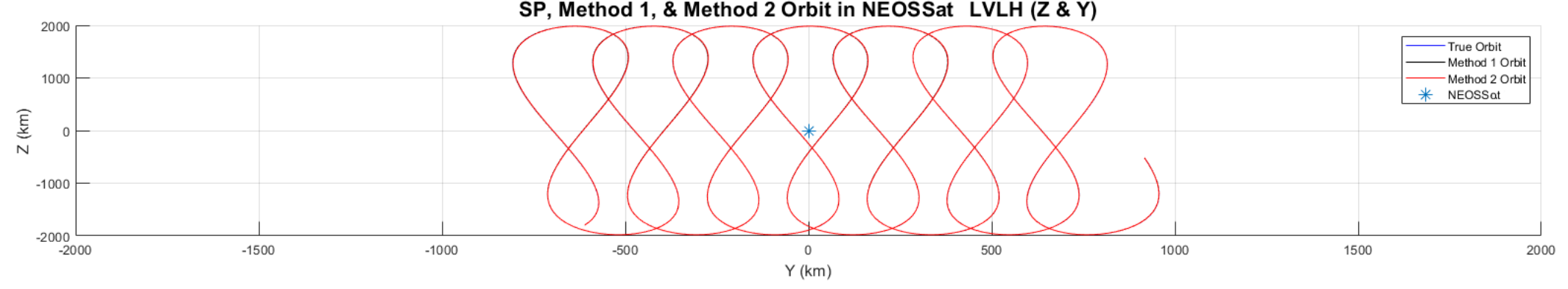

Figure 5.38: QuikScat Opportunity \#1 LVLH 


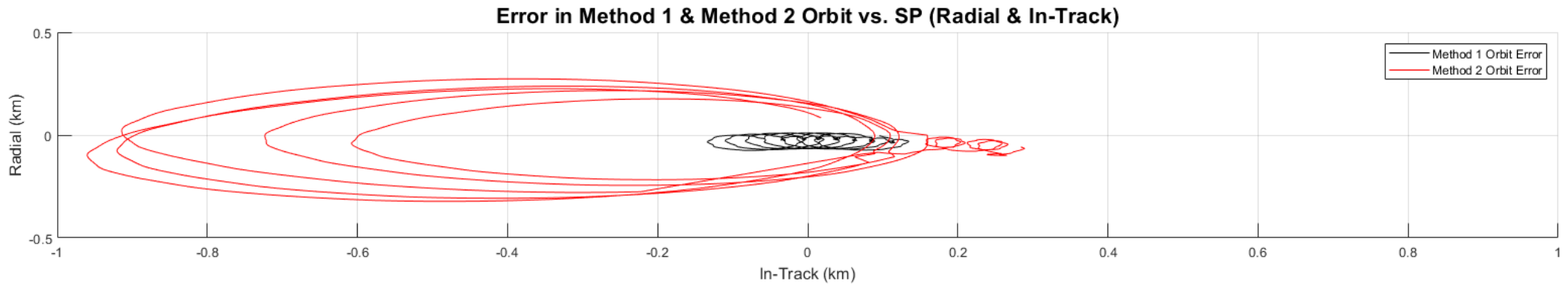

Error in Method 1 \& Method 2 Orbit vs. SP (Radial \& Cross-Track)

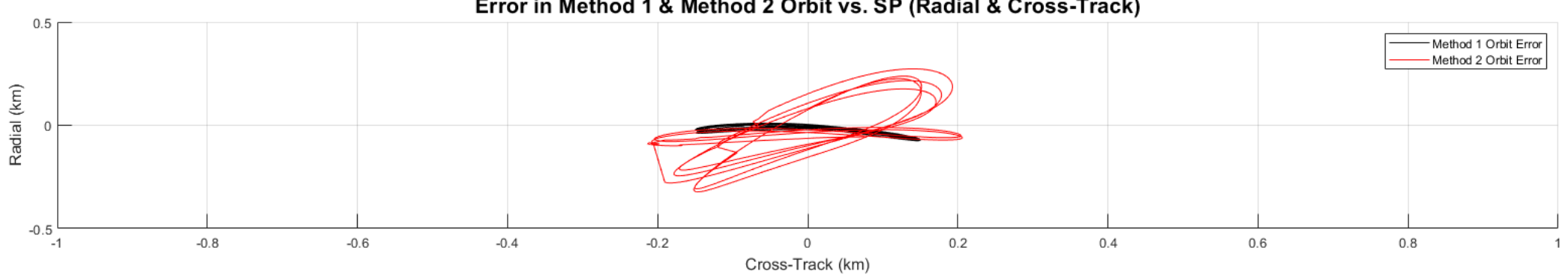

Error in Method $1 \&$ Method 2 Orbit vs. SP (Cross-Track \& In-Track)

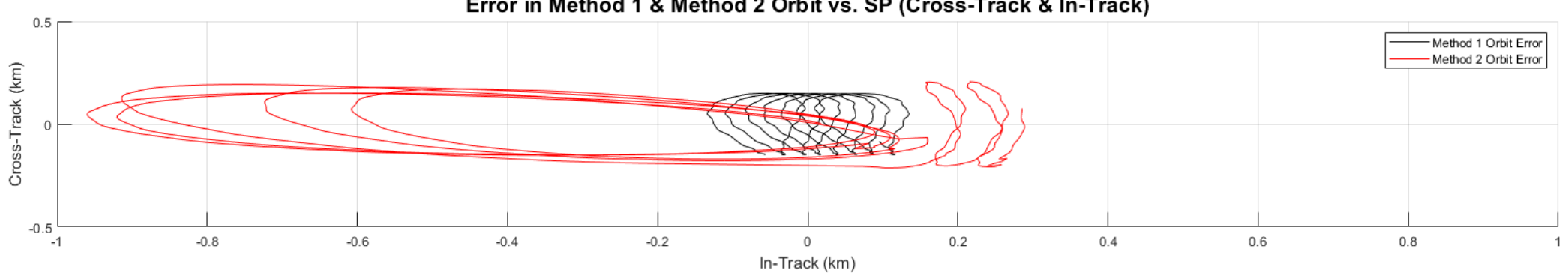

Figure 5.39: QuikScat Opportunity \#1 Orbit Estimate Errors 


\subsection{Sapphire}

The proximity event with Sapphire had 6 intervals which allowed sub-90 arcsec/sec angular rate tracks with respect to NEOSSat. The first interval started at 04:12:00 UTC on 12 Jun 2018 and the final interval ended at 12:41:55 on the same day. Within these intervals, NEOSSat was able to track and collect 55 observations, each observation containing a pair of RA and DEC measurements. During this particular event, the observations were collected on the passes as seen in Fig. 5.40 and in accordance with Table 5.7.

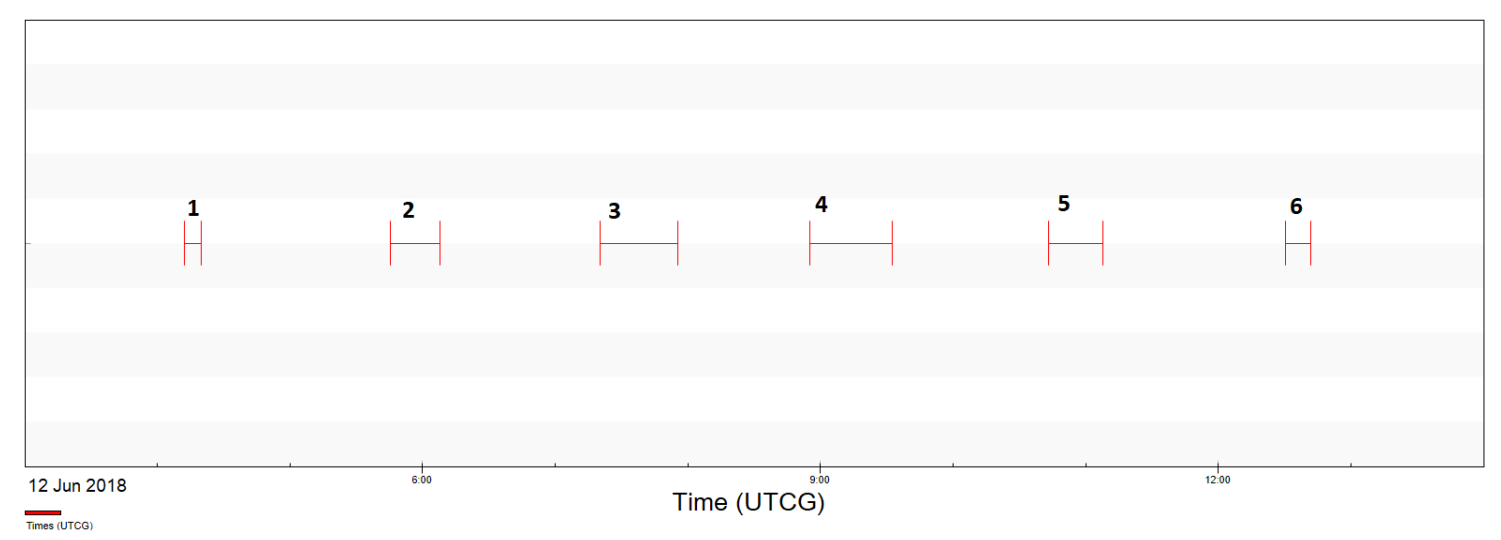

Figure 5.40: Sapphire observation intervals from NEOSSat

Table 5.7: Sapphire Observations

\begin{tabular}{ccccc}
\hline \hline Interval \# & Date & Start Time (UTC) & End Time (UTC) & \# of Observations \\
\hline \hline 1 & 12 Jun & $04: 12: 01$ & $04: 14: 01$ & 5 \\
2 & 12 Jun & $05: 50: 01$ & $06: 02: 01$ & 12 \\
3 & 12 Jun & $07: 22: 31$ & $07: 43: 01$ & 15 \\
4 & 12 Jun & $08: 58: 01$ & $09: 30: 01$ & 19 \\
5 & 12 Jun & $10: 45: 31$ & $10: 47: 01$ & 3 \\
6 & 12 Jun & $12: 30: 31$ & $12: 30: 31$ & 1 \\
\hline \hline
\end{tabular}

\subsubsection{Method 1}

Although a large number of observations were collected on multiple intervals, generating an orbit on Sapphire using Method 1 proved impossible. Over the course of 
observation, the satellite varied in distance from 20-50 km with respect to NEOSSat; an incredibly small separation for orbiting satellites, particularly for space surveillance purposes. Due to the close proximity of the satellite, the CCD on NEOSSat was saturated in every single image, leading to significantly larger object centroids, consistently increasing the associated error in the calculated object position when undergoing image processing. A sample image can be seen in Figs. 5.41 and 5.42.

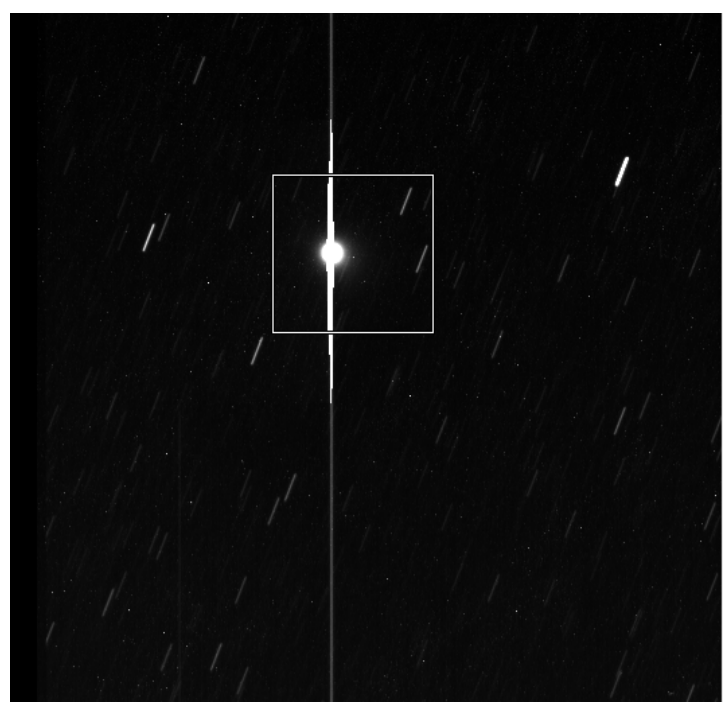

Figure 5.41: Saturated CCD Image of Sapphire as detected by NEOSSat at a separation of $32 \mathrm{~km}$

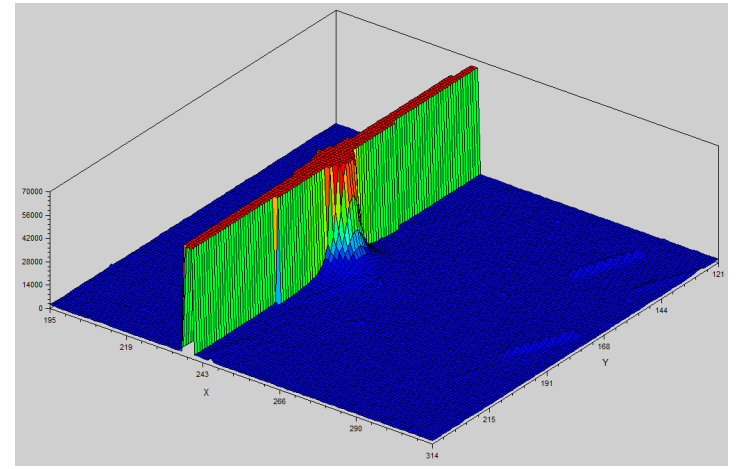

Figure 5.42: Saturated CCD Pixels of Sapphire as detected by NEOSSat at a separation of $32 \mathrm{~km}$

On multiple attempts, an IOD could be performed on different observations and resulted in initial orbit estimates. Regardless of which IOD solution was used, no combination of observations would allow a DC to converge on an orbit solution. In each case, the process would reject observations deemed too noisy, or being outside the acceptable range from the estimate in order to be accepted. The primary cause for this issue was due to the nature of the angles-only measurements themselves. As the measurements are of angular displacement, a physical displacement at a closer range would result in a larger angular displacement than the corresponding displacement at a further distance. For example, a $10 \mathrm{~m}$ displacement at GEO, the normal observing range of NEOSSat, would result in a 0.06 arcesond angular displacement, while the same displacement would result in 103 arcseconds at a $20 \mathrm{~km}$ distance. This causes the DC process to be more susceptible to angles-only noise at this incredibly close 
proximity, and the most likely reason for the algorithm failing.

\subsubsection{Method 2}

Unlike Method 1, Method 2 did succeed. The seeded orbit state for the satellite came from a TLE with an epoch of 13:10:39 UTC on 11 Jun 2018, approximately 13 hours before the observations and given estimated orbit uncertainties of R:102 m, I:471 m, $\mathrm{C}: 126 \mathrm{~m}$ [16]. When running the filter with a rejection tolerance of $3-\sigma$, a significant number of observations were rejected. It required opening the filter to $6-\sigma$ to allow all the observations to be accepted. The RIC comparisons for the run can be seen in Fig. 5.43.

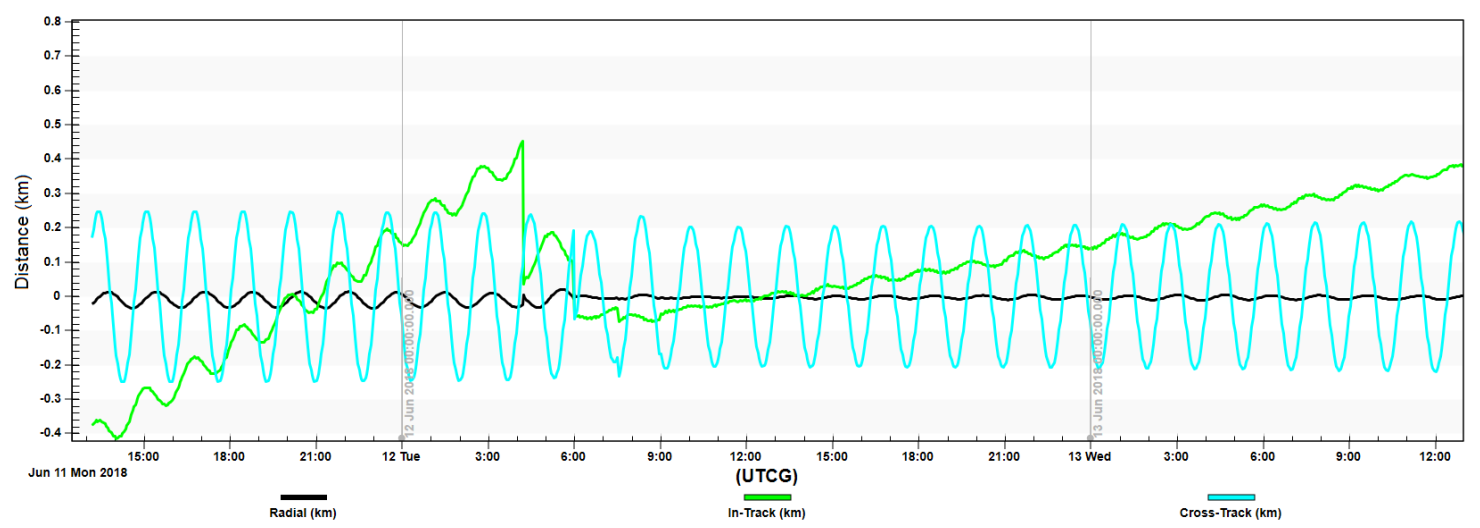

Figure 5.43: Sapphire Opportunity Method 2, 3- $\sigma$ filter RIC Comparison

Despite the presence of very noisy images, the resulting orbit fit for Sapphire was actually remarkably accurate. The in-track and radial position estimates were reduced from $400 \mathrm{~m}$ and $30 \mathrm{~m}$ to $75 \mathrm{~m}$ and $15 \mathrm{~m}$ respectively. Although there was a mis-match with the in-track velocity, this value was small and the in-track position error was still below one kilometer several days after the last measurement. Although adding complexity in the DC process, having the satellite so close did not overly affect the filtering process. Overall, there was remarkable accuracy in the radial component, a small mis-match of the cross-track component, and a slight mis-match of the in-track velocity. In contrast to several other satellite, opening up the filter covariance did not result in a significant increase in orbit accuracy, likely due to the proximity of the two satellite and the errors in angular position translating into physical displacements. In 
this case, due to the associated noise of the observations, best practice would be to cut off the filter acceptance at the 3 or $4-\sigma$ level.

\subsubsection{LVLH}

Due to the difficulties in generating inertial orbits with this event, no LVLH examination was undertaken.

\subsection{Discussion}

Despite the diversity in the intervals, some consistent findings could be observed and are outlined in the summary of the results in Table 5.8.

Table 5.8 outlines the difference in radial, in-track, and cross-track positions of the truth RSO positions to the most accurate of the orbit estimates 12 hours after the final observation was collected. The most consistent finding in each of the estimates is high accuracy in the radial position, consistently lower than $400 \mathrm{~m}$, and even as low as $5 \mathrm{~m}$. In-track errors could be found to be significant; however, this result was intermittent as it was dependant on the accuracy of the in-track velocity estimates. A consistent finding was that the cross-track errors were always more significant, but could be surpassed by in-track position errors, depending on velocity mis-match. This larger cross-track uncertainty, which lead to larger cross-track errors, was an expected result provided the viewing geometry between the satellite. As visualized in Fig. 4.10, NEOSSat limitations restricted observations to one section of the orbit, resulting in vantage points always looking along the cross-track axis. NEOSSat was unable to collect observations from other vantage points; ones that could have allowed for a reduction in the covariance along cross-track axis.

Although the limitation of NEOSSat performing observations from a cross-track vantage point led to larger errors along that axis, this issue can be extended to the in-track and radial components as well. If a satellite chose to perform co-orbital angles-only observations, the covariance of the orbit estimate will consistently be largest along the vector from the chaser to the target. 
Table 5.8: Results Summary

\begin{tabular}{ccccccc|cc|cc}
\hline \hline Object & Event \# & Method & $\begin{array}{c}\text { Min Range } \\
(\mathrm{km})\end{array}$ & $\begin{array}{c}\text { Max Range } \\
(\mathrm{km})\end{array}$ & \# of Obs & $\begin{array}{c}\text { Obs Time Span } \\
(\text { Hrs })\end{array}$ & $\begin{array}{c}\text { R } \\
(\mathrm{km})\end{array}$ & $\begin{array}{c}\text { I } \\
(\mathrm{km})\end{array}$ & $\begin{array}{c}\text { C } \\
(\mathrm{km})\end{array}$ & $\begin{array}{c}\text { RSS } \\
(\mathrm{km})\end{array}$ \\
\hline \hline BriteAustria & 1 & 1 & 101 & 180 & 22 & 0.49 & 0.005 & 0.278 & 0.705 & 0.758 \\
BriteAustria & 1 & 2 & 101 & 180 & 22 & 0.49 & 0.016 & 1.581 & 0.179 & 1.591 \\
\hline UniBrite & 1 & 1 & 76 & 130 & 32 & 2.05 & 0.389 & 2.970 & -4.52 & 5.422 \\
UniBrite & 1 & 2 & 76 & 130 & 32 & 2.05 & 0.066 & 0.665 & 0.284 & 0.726 \\
\hline AAUSAT3 & 1 & 1 & 130 & 240 & 28 & 3.43 & 0.382 & -10.758 & -6.048 & 12.347 \\
AAUSAT3 & 1 & 2 & 130 & 240 & 28 & 3.43 & 0.103 & 0.713 & 0.181 & 0.743 \\
\hline SARAL & 1 & 1 & 1062 & 2070 & 60 & 20.12 & 0.073 & 1.094 & 1.331 & 1.724 \\
SARAL & 1 & 2 & 1062 & 2070 & 60 & 20.12 & 0.114 & 1.141 & 0.483 & 1.244 \\
\hline AAUSAT3 & 2 & 1 & 240 & 254 & 6 & 0.06 & - & - & - & - \\
AAUSAT3 & 2 & 2 & 240 & 254 & 6 & 0.06 & 0.056 & -2.356 & 0.264 & 2.371 \\
\hline BriteAustria & 2 & 1 & 179 & 187 & 12 & 1.74 & - & - & - & - \\
BriteAustria & 2 & 2 & 179 & 187 & 12 & 1.74 & 0.009 & 0.111 & 0.332 & 0.350 \\
\hline QuikScat & 1 & 1 & 1250 & 2050 & 36 & 11.70 & 0.053 & 0.364 & 0.143 & 0.395 \\
QuikScat & 1 & 2 & 1250 & 2050 & 36 & 11.70 & 0.013 & 0.628 & 0.247 & 0.675 \\
\hline Sapphire & 1 & 1 & 20 & 50 & 55 & 8.31 & - & - & - & - \\
Sapphire & 1 & 2 & 20 & 50 & 55 & 8.31 & 0.009 & 0.146 & 0.206 & 0.253 \\
\hline \hline
\end{tabular}




\section{Chapter 6}

\section{Conclusion}

\subsection{Introduction}

This chapter presents a brief review of the objectives of this thesis, the summary of the findings in accordance with the thesis objectives outlined in Section 1.4, as well as recommendations for future work.

\subsection{Objectives Review}

This thesis was undertaken with the objective to investigate the feasibility and associated challenges of repurposing an optical space-based surveillance platfrom, the NEOSSat microsatellite, for applications in determining the relative orbit of a neighbouring target satellite. From that objective, six sub-objectives were identified:

- Confirm if NEOSSat is able to successfully acquire sufficient observation data from an object in proximity $(<2000 \mathrm{~km}$ range)

- Determine if the acquired observation data contains sufficient meaningful information required to generate an inertial orbit

- Determine if there is an optimal observation time-spacing to generate a relative orbit for an object in proximity to NEOSSat

- Determine if the calculated inertial orbit can be transformed to the relative reference frame for exploitation

- Assess the accuracy of the resulting inertial orbit against high-precision orbit ephemerides

- Assess the feasibility of implementing this methodology on future space surveillance spacecraft 


\subsection{Summary of Findings}

The findings of this work can be divided as they relate to the six sub-objectives re-iterated in Section 6.2.

- Confirm if NEOSSat is able to successfully acquire sufficient observation data from an object in proximity $(<2000 \mathrm{~km}$ range)

NEOSSat was successfully able to collect a sufficient number of observations on a variety of satellites. It was impossible to perform an IOD on two satellites, and this result was due to an insufficient time-spacing of those observations. There was also difficulty in performing a DC on the Sapphire observations, however, the number of observations was not the issue in this case, but the quality of those measurements.

\section{- Determine if the acquired observation data contains sufficient mean- ingful information required to generate an inertial orbit}

A significant finding from this thesis is the assessed metric accuracy of the NEOSSat sensor when including all steps of the data/image processing chain. From previously characterized noise for GPS satellites, the associated measurement noise of the sensor is effectively doubled. The assessed sources leading to this increased noise were the generally larger centroids of the observed objects in imagery, the increased angular sensitivity to position errors at closer imaging ranges, as well as the small sample size of images utilized for the assessment.

Fortunately, due to slew rate limitations, the region of the orbit where imaging operations occurred actually avoided issues regarding observability. The physical separation of the satellite in the cross-track component meant that the two orbit planes were slightly offset, limiting the issues related to observability outlined in Section 2.5.

None of the observations were on satellites that were purely co-planar; and as such, each was taken under geometries that minimized or eliminated observability issues. Overall, all of the proximity events encountered enabled varying levels of success; and was primarily dependent on the number of observations available and the associated spacing of those observations for IOD and DC processes to be carried 
out. The one exception to this success was the event with Sapphire. There were consistent issues encountered when trying to perform the DC process, regardless of IOD configuration, and the process would consistently diverge. The root cause identified was the increased brightness and noise of the observations at such close proximity that allowed for a consistent decrease in observation accuracy. This suggests that there is a limit to how close to the target spacecraft that these types of operations can be performed. Spacecraft in the ranges $\geq 100 \mathrm{~km}$ generally did not pose a major concern; Sapphire, imaged at consistent sub-100 km distances and even once coming within $10 \mathrm{~km}$ of NEOSSat, did pose a concern.

- Determine if there is an optimal observation time-spacing to generate a relative orbit for an object in proximity to NEOSSat

There is an optimal time-spacing, and it is due to the Gooding Angles Only IOD method used in this work. The algorithm requires a minimum $1 / 2$ to $1 / 3$ orbit revolution in order to successfully solve. Additionally, a multi-orbit time-span is less desirable due to the possibility of multiple solutions. As a result, the optimal timespan of observations is to be well spaced over one orbit in order to provide the best initial orbit estimate.

- Determine if the calculated inertial orbit can be transformed to the relative reference frame for exploitation

In every case where an inertial orbit was able to be generated, either through Method 1 or Method 2, the resulting orbit could be easily transformed into the LVLH frame due to the simple transformation outlined in Section 2.4.

- Assess the accuracy of the resulting inertial orbit against high-precision orbit ephemerides

Due to system constraints with NEOSSat, imaging operations were consistently carried out in a particular region of the orbit. Orbit determination solely using observations within this region would theoretically decrease the overall accuracy of the orbit estimate. Small errors in the resulting orbit fit, when limited to this span, 
do not necessarily allow for the corresponding accuracy in other regions of the orbit where the slight mis-matches could result in possible kilometer-scale position errors.

Due to the angles-only measurements collected, the associated range uncertainty was consistently an issue in each orbit fit for each satellite. This uncertainty, particularly for Method 1, resulted in large cross-track uncertainties due to that axis being largely parallel to the LOS vector between satellites. This problem is inherent with angles-only observations, and can prove problematic when greater range accuracy is desired for proximity operations. In order to minimize this problem, Method 2 was designed to allow the ingestion of a-priori state information within an EKF. By using the initial state, generated by a network of sensors with different vantage points, there would be a lower cross-track uncertainty than what would be generated by a single sensor with a sub-optimal vantage point.

When implementing Method 2, there was a consistent finding that the associated orbit uncertainties were too small when used to establish EKFs with a 3- $\sigma$ rejection tolerance. Initially, it was believed that the measurements themselves were too inaccurate, however, when comparing the different methods to the truth ephemerides, it was found that when including all the observations that would normally be rejected by a 3- $\sigma$ filter, actually tightened the orbit estimates to the truth values from the SP ephemerides. This issue appeared consistently where the filter needed to be opened to allow nearly all observations. Operational decisions to open this filter if implemented for real-world operation would certainly set a requirement for having high confidence in the sensor and the processing chain. Alternatively, a weight could be applied to images where images with poor optical characteristics would receive a low weight while images with good optical characteristics compared to a baseline image would receive a higher weight, biasing the fitting process to better observations.

\section{- Assess the feasibility of implementing this methodology on future space surveillance spacecraft}

In order to implement this type of operation on a space-based surveillance sensor, several significant hurdles would need to be overcome. The first of which would be the ability to greatly increase the tracking speed of the platform to increase the region 
of the orbit in which it could observe another spacecraft. If it is desired to perform automated relative orbit flight, the ability to perform image processing and orbit determination in a rapid manner would be critical. Due to the uncertainty in the orbit estimate, the use of the covariance as a decision tool for how close to get to the observed object, in contrast to absolute distance, could be established. Despite the growing trends in industry to increase the computational power onboard spacecraft, the associated overhead of this added functionality for a space-based surveillance satellite is possibly prohibitive. Nonetheless, interesting future experimentation on the viability of this approach is feasible.

\subsection{Recommendations for Future Work}

Several interesting areas for future work and research have been identified:

1. In-depth SARAL metric accuracy campaign: An in-depth metric assessment campaign could be carried out on SARAL. While briefly examined in this thesis, the number of associated observations were minimal when compared to the GPS calibration campaign. A more rigorous campaign evaluating both $1 \times 1$ and the $2 \times 2$ binning modes could be performed. As SARAL is observable from NEOSSat approximately every six weeks, the available high-precision ephemerides makes this a viable and feasible effort that could be completed over the course of several months to a year.

2. Sapphire follow-on: Provided the significant difficulty estimating Sapphire's orbit in this campaign, another opportunity to image this satellite while implementing the lessons-learned from the experience in 2018 could help to confirm the cause of the orbit fitting problem. Decreasing the noise of the observations would confirm observations taken at that incredibly small separation do indeed allow for proper inertial orbits to be estimated or if RSOs at those close distances have orbit normals sufficiently aligned to result in observability issues.

3. Leader-follower observations: Since the observation campaign was carried out, 
NEOSSat has undertaken a system modification that allows for up to 220 arcsec/sec slew rates to be performed. This capability increase dramatically expands the types and orbital configurations of other objects to be imaged by NEOSSat. This capability allows for objects to be tracked in-front and behind the satellite if treated as a true leader-follower pair. If observations are taken in this configuration, issues associated with observability would likely be significantly increased due to the LOS vectors being aligned within the satellite orbit planes.

4. Sensor Fusion: This work has focussed on using observations from a single chaser on a single target. If simultaneous observations from multiple chasers on a single target were used in combination, it would result in different orbit fits. Simultaneous observations from chasers with different LOS vectors to the target satellite would likely decrease the covariances provided the correct viewing geometries. To the author's knowledge, there are no active satellites which could readily support this study, however, it is an effort which could be achieved by means of simulation.

\subsection{A Final Note}

With the continued exploitation of the space domain in the field of exploration and experimentation, challenges associated with proximity operations will persist. This work serves as a preliminary assessment on the viability of using inertial orbit determination methods for exploitation in proximity operations. In the course of that assessment, several areas have been identified for additional investigation; there will always be niches in this field warranting examination and further thought. It is the

hope of this work's author that another will take up from where this work leaves off and attempts to push humanity's understanding a little bit further. 


\section{Bibliography}

[1] Woffinden, D. and Geller, D., "Observability Criteria for Angles-Only Navigation," IEEE Transactions on Aerospace and Electronic Systems, Vol. 45, No. 3, 2009, pp. 1194-1208.

[2] Geller, D. and Lovell, A., "Angles-Only Initial Relative Orbit Determination Performance Analysis using Cylindrical Coordinates," The Journal of the Astronautical Sciences, Vol. 64, 2017, pp. 72-96.

[3] Bonin, G., Roth, N., Armitage, S., Newman, J., Risi, B., and Zee, R., "CanX-4 and CanX-5 Precision Formation Flight: Mission Accomplished!" 29th Annual AIAA/USU Conference on Small Satellites, Logan Utah, United States, 2015.

[4] Sturdevant, R., "From Satellite Tracking to Space Situational Awareness: The USAF and Space Surveillance, 1957-2007," Air Power History, Vol. 55, No. 4, 2008, pp. 5-23.

[5] Gong, B., Geller, D., and Luo, J., "Initial Relative Orbit Determination Analytical Covariance and Performance Analysis for Proximity Operations," Journal of Spacecraft and Rockets, Vol. 53, No. 5, 2016, pp. 822-835.

[6] Vallado, D., Fundamentals of Astrodynamics and Applications, 2nd Ed, Microcosm Press, El Segundo, California, 2004.

[7] Tapley, B., Schutz, B., and Born, G., Statistical Orbit Determination, Elsevier Academic Press, Burlington, Massachusetts, 2004.

[8] Wright, J. and Chuba, B., Effective Use of Gooding IOD, Analytical Graphics Inc., 2004.

[9] Aldrin, E., Line-Of-Sight Guidance Techniques for Manned Orbital Rendezvous, Ph.D. thesis, Massachusetts Institute of Technology, 1963.

[10] Newman, J., Drift Recovery and Station Keeping for the CanX-4 8 CanX-5 Nanosatellite Formation Flying Mission, Master's thesis, 2015, University of Toronto.

[11] Woffinden, D., Angles-Only Navigation for Autonomous Rendezvous, Ph.D. thesis, Utah State University, 2008.

[12] Geller, D. and Klein, I., "Angles-Only Navigation State Observability During Orbital Proximity Operations," Journal of Guidance, Control, and Dynamics, Vol. 37, No. 6, 2014, pp. 1976-1983. 
[13] Geller, D. and Perez, A., "Initial Relative Orbit Determination for Close-in Proximity Operations," Journal of Guidance, Control, and Dynamics, Vol. 38, No. 9, 2015, pp. 1833-1841.

[14] Vetter, J., "Fifty Years of Orbit Determination: Development of Modern Astrodynamics Methods," Johns Hopkins APL Technical Digest, Vol. 27, No. 3, 2007, pp. 239-252.

[15] Vallado, R., Crawford, P., Hujsak, R., and Kelso, T., Revisiting Spacetrack Report \#3: Rev 2, Center for Space Standards and Innovation, 2006.

[16] T. Flohrer, H. K. and Klinkrad, H., "Assessment and Categorization of TLE Orbit Errors for the US SSN Catalogue," 2008 Advanced Maui Optical and Space Surveillance Technologies Conference (AMOS), Kihei, Maui, Hawaii, United States, 2008.

[17] Thorsteinson, S., Space Surveillance from a Microsatellite, Master's thesis, 2017, Royal Military College of Canada.

[18] Law, B., Africano, J., Hamada, K., Sydney, P., Hoo, V. S., Hoo, T. S., Barros, J., Nishimoto, D., OConnell, D., and Kervin, P., "The Phoenix Telescope at AMOS: Return Of The Baker-Nunn Camera," 2002 Astronomical Telescopes and Instrumentation Conference, Waikoloa Hawai'i, United States, 2002.

[19] Gaposchkin, M., von Braun, C., and Sharma, J., "Space-Based Space Surveillance with the Space-Based Visible," Journal of Guidance, Control, and Dynamics, Vol. 23, No. 1, 2000, pp. 148-152.

[20] Maskell, P. and Oram, L., "Sapphire: Canadas Answer to Space-Based Surveillance of Orbital Objects," 2008 Advanced Maui Optical and Space Surveillance Technologies Conference (AMOS), Wailea, Maui, Hawaii, United States, 2008.

[21] Wallace, B., Pinkney, F., Scott, R., Bedard, D., and et al., J. R., "The Near Earth Object Surveillance Satellite (NEOSSat)," 55th International Astronautical Congress 2004, Vancouver, Canada, 2004.

[22] Bedard, D., Scott, L., Wallace, B., and et al., "Risk Reduction Activities for the Near-Earth Object Surveillance Satellite Project," 2006 Advanced Maui Optical and Space Surveillance Technologies Conference (AMOS), Kihei, Maui, Hawaii, United States, 2006.

[23] Clohessy, W. and Wiltshire, R., "Terminal Guidance System for Satellite Rendezvous," Journal of the Aero/Space Sciences, Vol. 27, No. 9, 1960, pp. 653-658.

[24] Ulrich, S., "AERO3240 Orbital Mechanics," 2017, Undergraduate Course Notes. 
[25] Ulrich, S., Direct Adaptive Control Methodologies for Flexible-Joint Space Manipulators with Uncertainties and Modeling Errors, Ph.D. thesis, Carleton University, 2012.

[26] Gaias, G., D’Amico, S., and Ardaens, J.-S., "Angles-Only Navigation to a Noncooperative Satellite Using Relative Orbital Elements," Journal of Guidance, Control, and Dynamics, Vol. 37, No. 2, 2014, pp. 439-451.

[27] Vallado, D., "Evaluating Gooding Angles-only Orbit Determination of Space Based Space Surveillance Measurements," 012010.

[28] Tombasco, J., Orbit Estimation of Geosynchronous Objects Via Ground-Based and Space-Based Optical Tracking, Ph.D. thesis, University of Colorado, 2011.

[29] Ricklefs, R., Consolidated Laser Ranging Prediction Format Version 1.01, International Laser Ranging Service, February 2006.

[30] Zelensky, N. and et al., F. L., "Towards the 1-cm SARAL orbit," Advances in Space Research, Vol. 58, 2016, pp. 2651-2676.

[31] Scott, R. and et al., B. W., "Toward Microsatellite Based Space Situational Awareness," 2013 Advanced Maui Optical and Space Surveillance Technologies Conference (AMOS), Wailea, Maui, Hawaii, United States, 2013. 


\section{Appendix A}

\section{Data Formats}

This appendix provides an outline of different data formats used in support of work on this thesis.

\section{A.1 ILRS Standard Product 3 Format Ephemeris}

Figure A.1 outlines the top portion of a SARAL Standard Product 3 Format ephemeris file. The Standard Product 3 Format file consists of ephemeris points including time, position, and velocity in the International Terrestrial Reference Frame over the span of approximately one week.

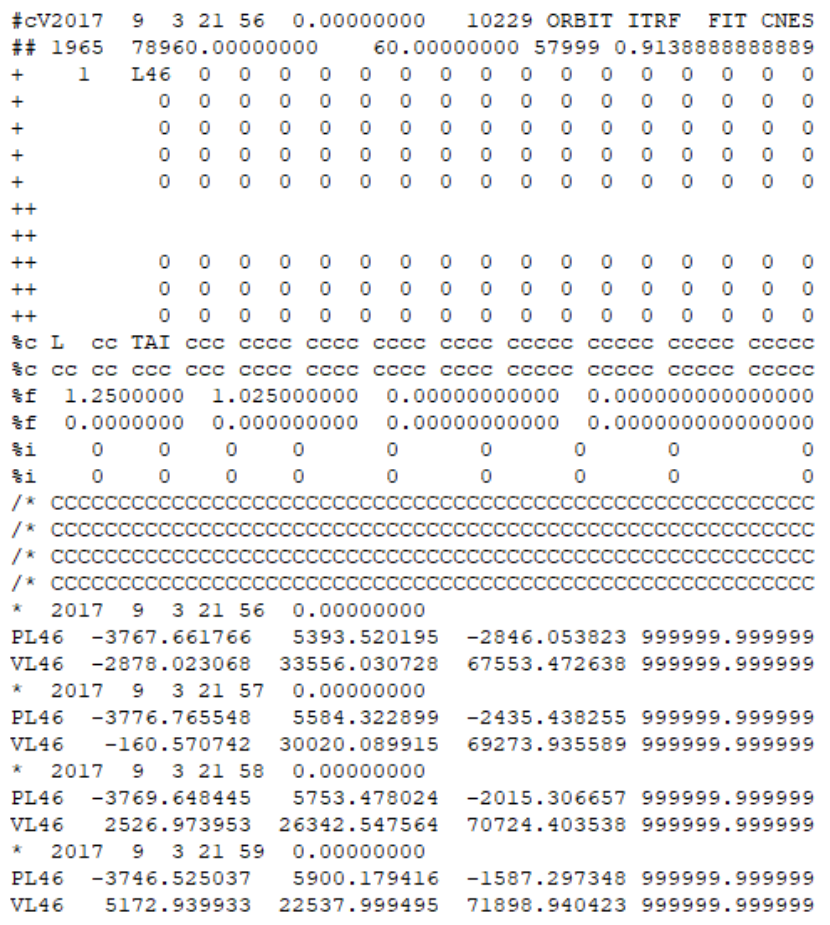

Figure A.1: SARAL Standard Product 3 Format ephemeris from the Centre National d'Études Spatiales 


\section{A.2 STK Ephemerides}

Figure A.2 outlines the top portion of a SARAL STK ephemeris file. The STK file consists of ephemeris points including time, position, and velocity in the International Terrestrial Reference Frame over the span of approximately one week.

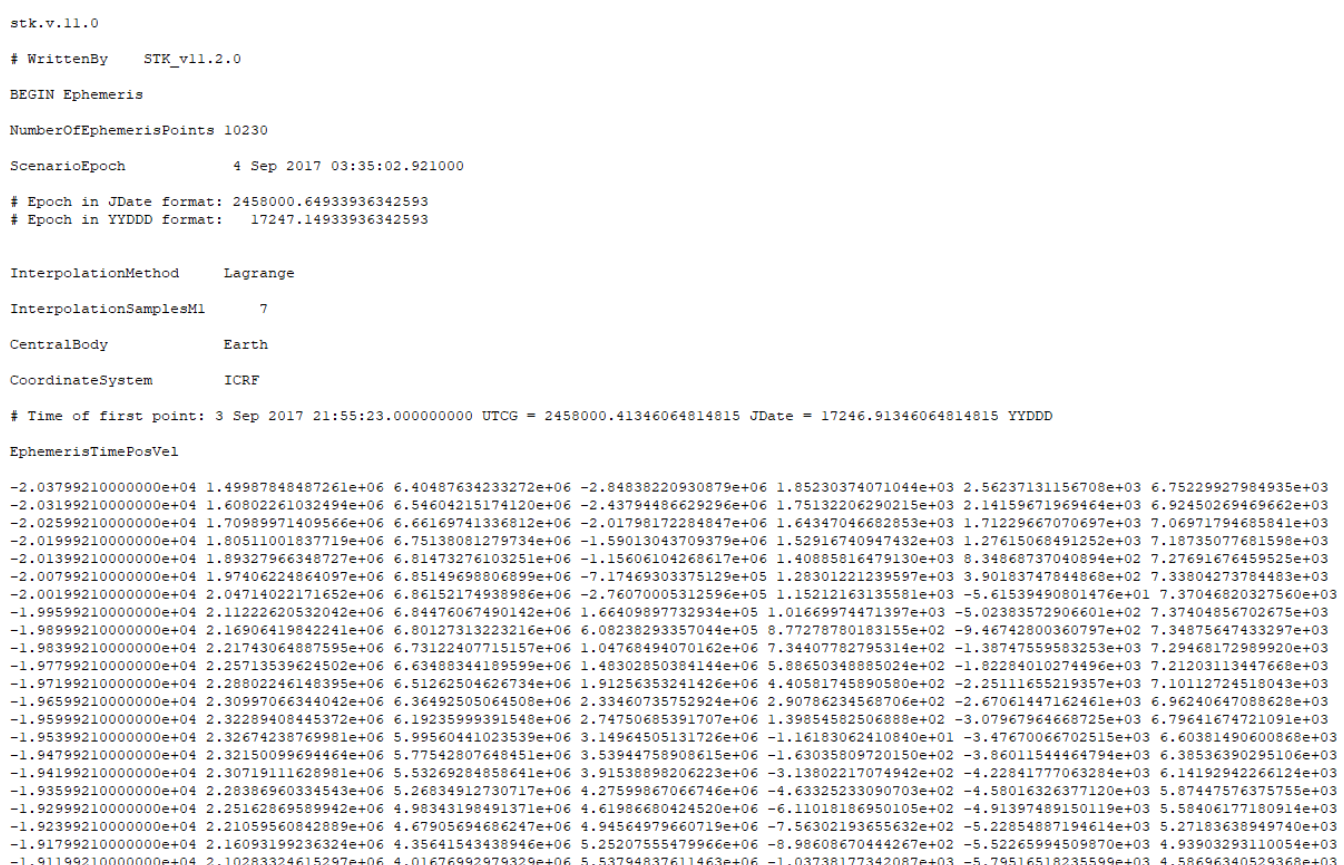

Figure A.2: SARAL Ephemeris in STK Format 


\section{Appendix B}

\section{ODTK Settings}

This appendix provides an outline of the major ODTK force model settings utilized in support of work on this thesis. These settings were used generically for each event, while specific parameter settings such as mass and surface area varied for individual satellites.

Table B.1: ODTK Settings

\begin{tabular}{cc}
\hline \hline Parameter & Setting \\
\hline \hline Gravity Model & EGM2008 \\
Nutation Method & IERS1996 \\
Ionospheric Model & IRI2007 \\
Atmospheric Density Model & CIRA 1972 \\
Object Physical Model & Spherical \\
Drag Coefficient (Cd) & 2.2 \\
Propagation Method & RKF 7(8) \\
\hline
\end{tabular}

